TOPICAL REVIEW

\title{
Functionalized carbon nanotubes and device applications
}

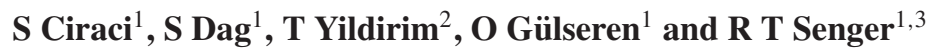 \\ ${ }^{1}$ Department of Physics, Bilkent University, 06800 Ankara, Turkey \\ ${ }^{2}$ NIST Center for Neutron Research, National Institute of Standards and Technology, \\ Gaithersburg, MD 20899, USA \\ 3 TÜBİTAK-UEKAE, P.K. 74, 41470 Gebze, Kocaeli, Turkey \\ E-mail: ciraci@fen.bilkent.edu.tr
}

Received 8 March 2004

Published 9 July 2004

Online at stacks.iop.org/JPhysCM/16/R901

doi:10.1088/0953-8984/16/29/R01

\begin{abstract}
Carbon nanotubes, in which the two-dimensional hexagonal lattice of graphene is transformed into a quasi-one-dimensional lattice by conserving the local bond arrangement, provide several structural parameters for engineering novel physical properties suitable for ultimate miniaturization. Recent interest in nanoscience and nanotechnology has driven a tremendous research activity in carbon nanotubes, which has dealt with a variety of problems and produced a number of new results. Most of the effort has gone into revealing various physical properties of nanotubes and functionalizing them in different ways. This paper covers a narrow region in this enormous research field and reviews only a limited number of recent studies which fit within its scope. First, we examine selected physical properties of bare carbon nanotubes, and then study how the mechanical and electronic properties of different tubes can be modified by radial strain, structural defects and adsorption of foreign atoms and molecules. Magnetization of carbon nanotubes by foreign atom adsorption has been of particular interest. Finally, we discuss specific device models as well as fabricated devices which exploit various properties of carbon nanotubes.
\end{abstract}

(Some figures in this article are in colour only in the electronic version)

\section{Contents}

1. Introduction $\quad 902$

2. Atomic and electronic structure 904

2.1. Geometric structure and energetics of bare SWNTs 904

2.2. Energy band structure 906

3. Hydrogenation of carbon nanotubes 908

0953-8984/04/290901+60\$30.00 @ 2004 IOP Publishing Ltd Printed in the UK R901 
4. Oxygenation of carbon nanotubes 912

4.1. Physisorption of $\mathrm{O}_{2}$ molecules 913

4.2. Chemisorption of oxygen atoms 916

5. Adsorption of individual atoms on SWNTs 917

5.1. Binding geometry and binding energies 919

5.2. Electronic structure $\quad 923$

5.3. Transition element covered or filled SWNTs 926

6. Radial deformation of carbon nanotubes 927

6.1. Elasticity 928

6.2. Effect on the electronic structure 930

6.3. Effect on the chemical reactivity 932

6.4. Effect of pressure on nanoropes 934

7. Devices based on carbon nanotubes 936

7.1. Ab initio methods in transport calculations 936

7.2. Device models $\quad 942$

8. Devices fabricated using carbon nanotubes 952

8.1. Transistors based on carbon nanotubes 952

8.2. Chemical sensors $\quad 955$

9. Conclusions 955

Acknowledgments $\quad 956$

References $\quad 956$

\section{Introduction}

Research on carbon nanotubes is ever intensifying in diverse fields of science and engineering in spite of the twelve years that have passed since its first discovery by Iijima [1]. There are several reasons that so much interest has been focused on these materials. First of all, carbon nanotubes have been a natural curb for several research programmes, which were tuned to $\mathrm{C}_{60}$ but all of a sudden came to an end without any great technological applications having been found. Secondly, researchers, who can touch and relocate individual atoms have been challenged to discover the novel properties of these strange materials in order to transform them into new devices or use them in other technological applications. As a result of the rapid rise in the speed, as well as the rapid reduction in the size of electronic devices, new paradigms will be needed to overcome the barriers set by the traditional technologies to produce ever smaller and faster devices. Extensive research dealing with the modification of electronic structure for desired device operations has indicated that carbon nanotubes can be considered as a new frontier in the search for the ultimate miniaturization of electronic circuits with ultrahigh density components and new functionalities. Several devices fabricated so far with different functionalities appear to meet the great expectations for carbon nanotubes.

Carbon nanotubes are unique materials, which offer a variety of structural parameters for engineering their physical and chemical properties [2,3]. They can be synthesized as singlewall (SWNT) or multiple-wall (MWNT) nanotubes; they can form ropes or even crystals. Even an ultimate one-dimensional carbon chain at the centre of a MWNT (and stabilized by the innermost nanotube) has been discovered in cathode deposits [4]. SWNTs are basically rolled graphite sheets, which are characterized by two integers $(n, m)$ defining the rolling (or chiral vector) $\mathbf{C}=n \mathbf{a}_{1}+m \mathbf{a}_{2}$, in terms of the two-dimensional (2D) hexagonal Bravais lattice vectors of graphene, $\mathbf{a}_{1}$ and $\mathbf{a}_{2}$. Then the radius of the tube is given in terms of $(n, m)$ by the relation $R=a_{0}\left(n^{2}+m^{2}+n m\right)^{1 / 2} / 2 \pi$, where $a_{0}=\left|\mathbf{a}_{1}\right|=\left|\mathbf{a}_{2}\right|$. SWNTs exhibit different electronic structures depending on $n$ and $m$ (i.e. on their chirality and radius). 
The mechanical properties of carbon nanotubes are striking. They are flexible and can sustain large elastic deformations radially; at the same time they are very strong axially with high yield strength $[5,6]$. Their strength far exceeds that of any other fibre. Even more striking is the response of the electronic structure to the radial deformation leading to dramatic changes. As has been predicted theoretically and confirmed experimentally, a semiconducting zigzag tube becomes metallic with finite state density at the Fermi level as a result of radial deformation transforming the circular cross section into an ellipse. At the same time the chemical activity of the surface of the tube undergoes a change; the interaction of adatoms with the SWNT occurs differently at high and low curvature sites. The metal-semiconductor transition induced by elastic deformation has important implications.

Physical and chemical properties of a SWNT can also be modified by the adsorption of foreign atoms or molecules. This process is usually named functionalization, and carries great potential in tailoring new nanostructures for engineering them according to a desired application. For example, depending on the pattern of hydrogen atom coverage, while a metallic armchair SWNT can be transformed to a wide band gap semiconductor, a semiconducting zigzag tube may become a metal with very high state density. A free SWNT, which is normally nonmagnetic, becomes magnetic with unpaired spins upon the adsorption of oxygen molecules or specific transition metal atoms. A recent study demonstrates that a semiconducting zigzag tube becomes both a magnetic and a high conductance wire as a result of Ti coating [7]. A selectable functionalization of the (5,5) $\mathrm{SWNT}$ resulting from $\mathrm{CH}_{n}(n=1-3)$ adsorption and decoration gives rise to a substantial change in the density of states [8]. Suitably doped carbon nanotubes can be functionalized by selectively forming chemical bonds with ligands at the chemically active impurity site [9]. Apparently, functionalization of carbon nanotubes, in particular biological and chemical functionalization, is an extensive field incorporating several recent studies in diverse fields. This review is confined rather to the functionalized SWNTs which have their electronic and magnetic properties modified.

Clearly, carbon nanotubes offer many parameters to deal with and many options for generating properties suitable for a desired functionality. One of the great challenges of research on carbon nanotubes has been the realization of nanometre-sized optoelectronic devices and nanomagnets. In an effort to discover new features of technological interest, several theoretical and experimental studies actively explored SWNTs, MWNTs, ropes and their functionalized forms, which resulted in many papers. However, due to its focus compounded with the space limitations, the scope of this review article has been kept necessarily limited. The subject matter that we have left out is in no way less significant than what we have included.

We followed a logical order that starts from fundamental aspects and ends with technological applications. We first established a background concerning the atomic and electronic structure of various SWNTs. We then examined various methods which are used to modify the properties of SWNTs to generate new nanostructures. Finally, we discussed how these properties have been used to model devices. The organization of the article is as follows. In section 2, we have presented a discussion of electronic structure obtained from firstprinciples calculations together with a comparison made to empirical studies. Hydrogenation and oxygenation of SWNTs have been dealt separately in sections 3 and 4, respectively, owing to the several recent papers in this area, as well as due to the relevance to hydrogen storage. Section 5 has been be devoted to the individual adsorption of 24 different atoms (ranging from alkali and simple metal atoms to group IV atoms and most transition metal atoms), where their binding structures and binding energies, and the effect of their adsorption on the electronic structure, have been investigated. Since the ground state for most of the transition metal atoms adsorbed on the surface of SWNTs is magnetic, and hence has net spin, this section is important for the magnetic properties of functionalized nanotubes. In section 6 , we have investigated the 
effects of radial deformation, and have examined how the electronic and chemical properties are modified. Here, the atomic structure, elasticity and electronic structure and binding of adatoms under radial deformation have been discussed. In section 7, we have reviewed recent developments in transport theory and have presented some device models developed using the features and physical properties discussed in previous sections. Section 8 reviews briefly the recent progress made in the fabrication of electronic devices based on carbon nanotubes and has described a few such devices produced. The paper is concluded with outlooks and prospective developments.

\section{Atomic and electronic structure}

The electronic band structure of SWNTs can be deduced by mapping the band structure of graphene in a 2D hexagonal lattice onto a cylinder [3, 10-14]. In this respect, the SWNT presents an interesting example, in which dimensionality is reduced from two to one. The analysis based on the band folding indicates that the $(n, n)$ armchair nanotubes are always metal with $\pi^{*}$ conduction, and $\pi$ valence bands crossing at the Fermi level, and exhibit 1D quantum conduction $[14,15]$. The $(n, 0)$ zigzag SWNTs are generally semiconductor and are only metal if $n$ is an integer multiple of 3. Although the overall electronic structure of SWNTs has been described by this simple picture, recent studies $[16,18]$ have shown much more complicated structural dependence. For example, the $(9,0)$ tube is, in fact, a small band gap semiconductor. The semiconducting behaviour of SWNTs has been of particular interest, since the electronic properties can be controlled by doping or implementing defects in nanotube based optoelectronic devices [19-26].

Band calculations of SWNTs were initially performed by using a one-band $\pi$ orbital tight binding model [11]. Subsequently, experimental data [27-30] on the band gaps were extrapolated to confirm the inverse proportionality with the radius of the nanotube [13]. Later, first-principles calculation [31] within the local density approximation (LDA) showed that the $\sigma^{*}-\pi^{*}$ hybridization becomes significant at small $R$ (or at high curvature). Recent analytical studies [32-34] showed the importance of curvature effects in carbon nanotubes. Nonetheless, band calculations performed by using different methods have been at variance on the values of the band gap. Extensive theoretical analysis of the band structure of SWNTs together with the curvature effects on geometric and electronic structure has been carried out recently [18] by using first-principles pseudopotential plane wave method [35] calculations within the generalized gradient approximation (GGA) [36].

\subsection{Geometric structure and energetics of bare SWNTs}

Because of cylindrical symmetry, the structural parameters of SWNTs deviate from those of graphene. The inset to figure 1 shows a schematic side view of a zigzag SWNT which indicates two types of $\mathrm{C}-\mathrm{C}$ bonds $\left(d_{1}\right.$ and $\left.d_{2}\right)$ and $\mathrm{C}-\mathrm{C}-\mathrm{C}$ bond angles $\left(\Theta_{1}\right.$ and $\left.\Theta_{2}\right)$. The variations of the normalized bond lengths (i.e. $d_{1} / d_{0}$ and $d_{2} / d_{0}$ where $d_{0}$ is the optimized C-C bond length in graphene) and the bond angles with tube radius $R$ (or $n$ ) are shown in figures 1(a) and (b), respectively. Both the bond lengths and the bond angles display a monotonic variation and approach the graphene values as the radius increases. As pointed out earlier for the armchair SWNTs [37], the curvature effects, however, become significant at small radii. The zigzag bond angle $\left(\theta_{1}\right)$ decreases with decreasing radius. It is about $12^{\circ}$ less than $120^{\circ}$ for the $(4,0)$ SWNT, while the length of the corresponding zigzag bonds $\left(d_{2}\right)$ increases with decreasing $R$ and the length of the parallel bond $\left(d_{1}\right)$ decreases to a lesser extent with decreasing $R$. The angle involving this latter bond $\left(\theta_{2}\right)$ is almost constant. 

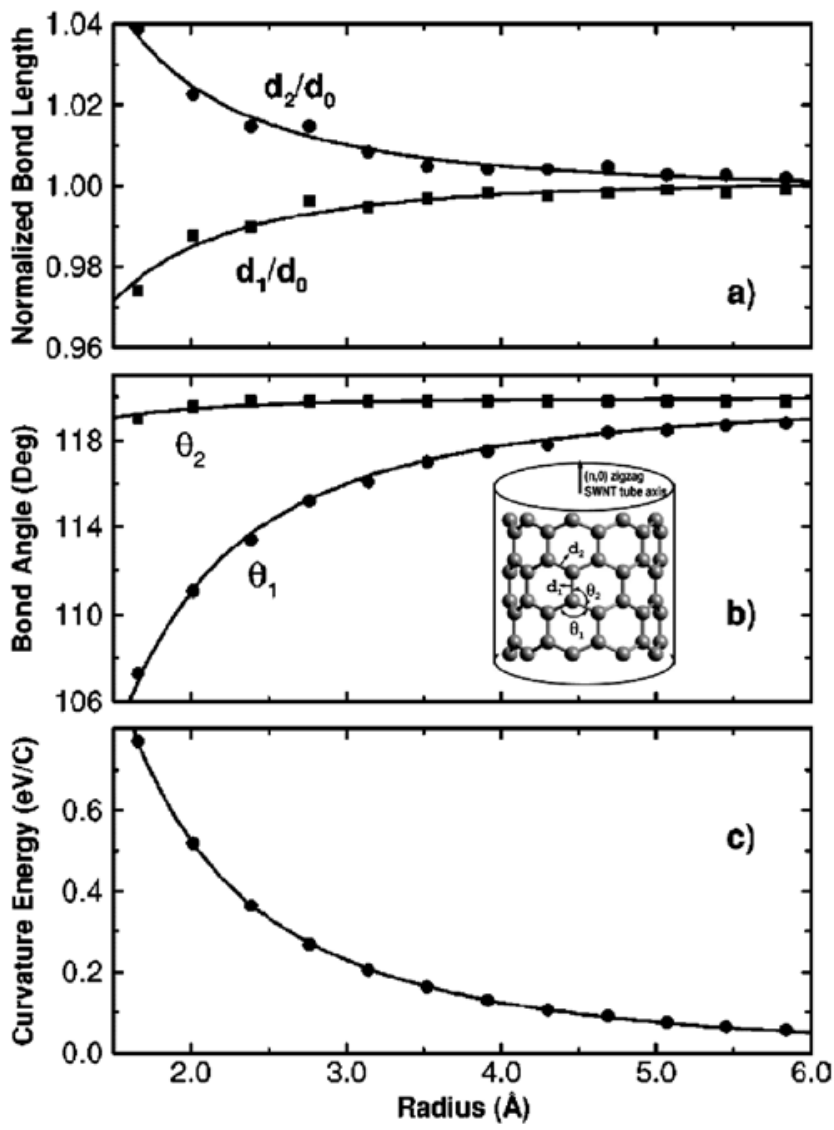

Figure 1. Inset: a schematic side view of a zigzag SWNT, indicating two types of $\mathrm{C}-\mathrm{C}$ bonds and C-C $-\mathrm{C}$ bond angles. These are labelled as $d_{1}, d_{2}, \theta_{1}$ and $\theta_{2}$. (a) Normalized bond lengths $\left(d_{1} / d_{0}\right.$ and $\left.d_{2} / d_{0}\right)$ versus the tube radius $R\left(d_{0}=1.41 \AA\right)$. (b) The bond angles $\left(\theta_{1}\right.$ and $\left.\theta_{2}\right)$ versus $R$. (c) The curvature energy, $E_{\text {cur }}$ per carbon atom with respect to graphene as a function of the tube radius. The solid curves are the fit to the data as $\alpha / R^{2}$. (Reproduced from [18].)

An internal strain is implemented upon the formation of tubular structure from the graphene sheet. The associated strain energy, which is specified as the curvature energy, $E_{\mathrm{cur}}$, is calculated as the difference of total energy per carbon atom between the bare SWNT and the graphene (i.e. $E_{\text {cur }}=E_{\mathrm{T}, \mathrm{SWNT}}-E_{\mathrm{T} \text {,graphene }}$ ) for $4 \leqslant n \leqslant 15$. The calculated curvature energies are shown in figure $1(\mathrm{c})$. As expected, $E_{\text {cur }}$ is positive and increases with increasing curvature. The cohesive energies of SWNTs are also curvature dependent, and are calculated from the expression $E_{\mathrm{coh}}=E_{\mathrm{T}}[\mathrm{C}]-E_{\mathrm{T}}[\mathrm{SWNT}]$ in terms of the total energy of the free carbon atom, and the total energy of a SWNT per carbon atom. For a zigzag tube, it is small for small $n$ and increases with $n$, and eventually saturates to the cohesive energy of graphene. Similar trends also exist for the armchair tubes. In classical theory of elasticity the curvature energy is given by the following expression [38-40]: $E_{\text {cur }}=\alpha / R^{2}$, where $\alpha=Y t^{3} \Omega / 24$. Here $Y$ is the Young's modulus, $t$ is the thickness of the tube, and $\Omega$ is the atomic volume. Interestingly, curvature energies obtained from first-principles calculations yield a perfect fit to the relation $\alpha / R^{2}$ as seen in figure 1 (c). In this fit $\alpha$ is found to be $2.14 \mathrm{eV} \AA^{2} /$ atom [18]. 


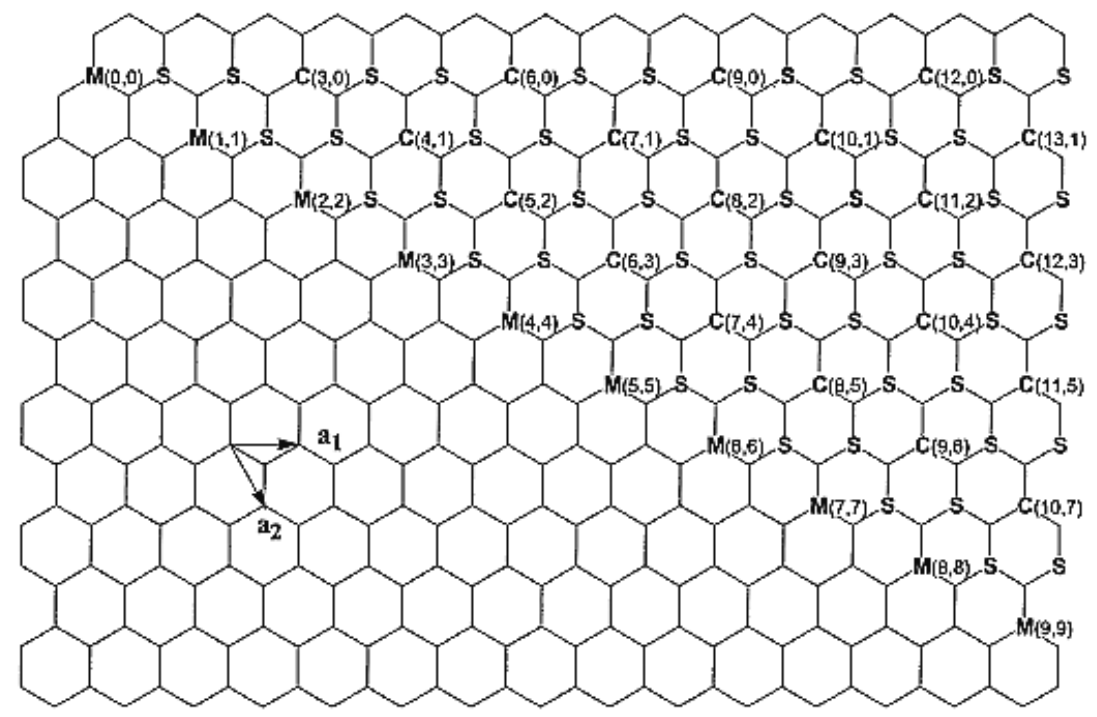

Figure 2. A map of chiral vectors that determine the chirality of SWNTs. Each vector is specified by the $(n, m)$ indices. $\mathrm{M}, \mathrm{S}$ and $\mathrm{C}$ denote metals, semiconductors and curvature induced small gap semiconducting SWNTs, respectively. (Reproduced from [17].)

Table 1. Band gap, $E_{\mathrm{g}}$, as a function of radius $R$ for $(n, 0)$ zigzag nanotubes. M denotes the metallic state. The first-row values were obtained within the GGA in [18]. The second and third rows from [31] give LDA results, while all the rest are tight binding (TB) results. The two rows from [42] are for two different TB parametrizations.

\begin{tabular}{|c|c|c|c|c|c|c|c|c|c|c|c|c|}
\hline$n$ & 4 & 5 & 6 & 7 & 8 & 9 & 10 & 11 & 12 & 13 & 14 & 15 \\
\hline$R(\AA)$ & 1.66 & 2.02 & 2.39 & 2.76 & 3.14 & 3.52 & 3.91 & 4.30 & 4.69 & 5.07 & 5.45 & 5.84 \\
\hline [18] & M & M & M & 0.243 & 0.643 & 0.093 & 0.764 & 0.939 & 0.078 & 0.625 & 0.736 & 0.028 \\
\hline [31] & & & M & 0.09 & 0.62 & 0.17 & & & & & & \\
\hline [31] & & & 0.05 & 1.04 & 1.19 & 0.07 & & & & & & \\
\hline [11] & & & 0.21 & 1.0 & 1.22 & 0.045 & 0.86 & 0.89 & 0.008 & 0.697 & 0.7 & 0.0 \\
\hline [42] & & & & 0.79 & 1.12 & & 0.65 & 0.80 & & & & \\
\hline [42] & & & & 1.11 & 1.33 & & 0.87 & 0.96 & & & & \\
\hline
\end{tabular}

\subsection{Energy band structure}

According to the zone folding scheme a $(n, m)$ SWNT has been predicted to be metallic when $n-m=3 \times$ integer, since the doubly degenerate $\pi$ and $\pi^{*}$ states, which overlap at the K point of the hexagonal Brillouin zone (BZ) of graphene, fold to the $\Gamma$ point of the tube $[11,12]$. Thus, to first order all $(n, n)$ armchair tubes are metallic, but $(n, 0)$ zigzag tubes become metallic when $n$ is a multiple of 3 . In figure 2, a map of the chiral vector specified by $(n, m)$ indices indicates whether a SWNT is a semiconductor or metal. This simple picture provides a qualitative understanding, but fails to describe some important features, in particular for small radius or metallic nanotubes. SWNTs specified by ' $\mathrm{C}$ ' in figure 2 form a subgroup of semiconducting tubes, which have a small band gap induced by the curvature. This situation is clearly shown in table 1 , where the band gaps $E_{\mathrm{g}}$ of $(n, 0)$ SWNTs calculated by different methods are compared.

First-principles GGA calculations [18] resulted in small, but nonzero energy band gaps of 93,78 and $28 \mathrm{meV}$ for $(9,0),(12,0)$ and $(15,0)$ SWNTs, respectively. These gaps 

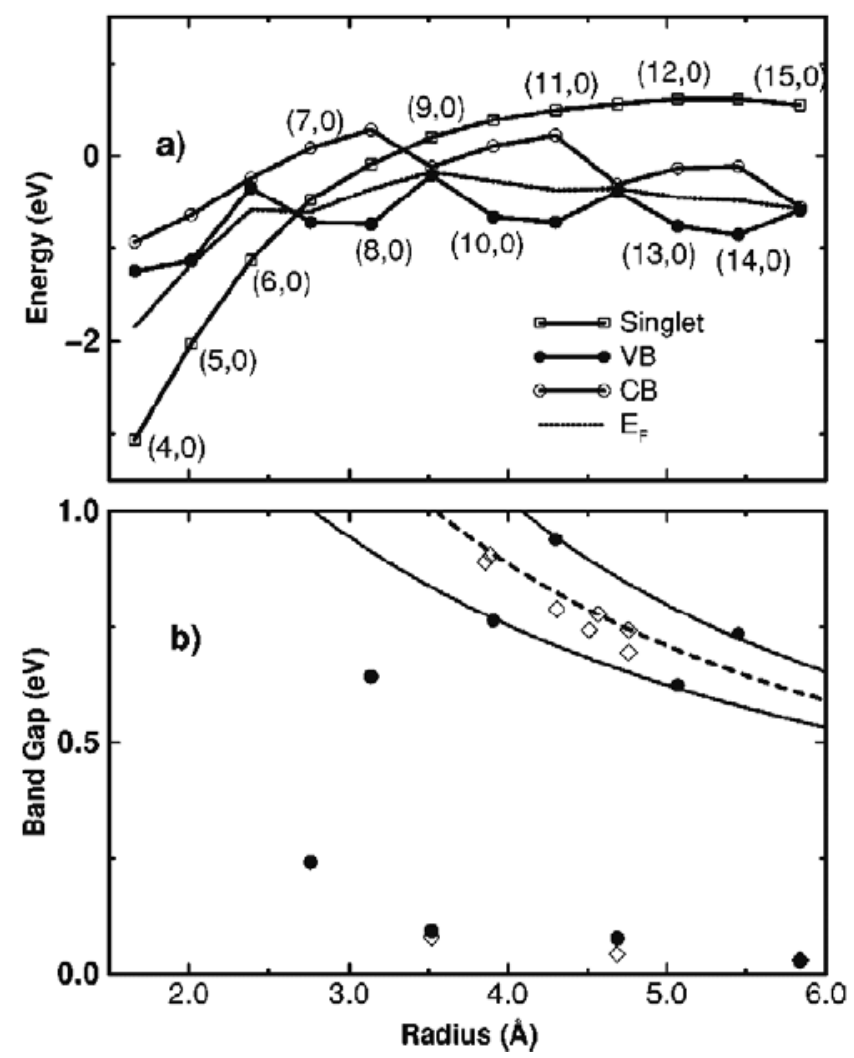

Figure 3. (a) Energies of the doubly degenerate $\pi$ states (VB), the doubly degenerate $\pi^{*}$ states (CB) and the singlet $\pi^{*}$ state as a function of nanotube radius. Each data point corresponds to $n$ ranging from 4 to 15 consecutively. (b) The calculated band gaps of [18] are shown by filled symbols. Solid (dashed) curves are the plots of equation (2) (equation (1)). The experimental data taken from [16, 29, 30] are shown by open diamonds. (Reproduced from [18].)

are measured by scanning tunnelling spectroscopy (STS) experiments [16] as 80, 42 and $29 \mathrm{meV}$, in the same order. Recently, Kim et al [17] synthesized ultralong and high percentage semiconducting SWNTs where further experimental evidence for the small band gap tubes was provided. The biggest discrepancy noted in table 1 is between the tight binding and the first-principles values of the gaps for small radius tubes such as $(7,0)$. These results indicate that curvature effects are important and the simple zone folding picture has to be improved. Moreover, the analysis of the LDA bands of the $(6,0)$ SWNT calculated by Blase et al [31] brought in another important effect of the curvature. The antibonding singlet $\pi^{*}$ and $\sigma^{*}$ states mix and repel each other in curved graphene. As a result, the purely $\pi^{*}$ state of planar graphene is lowered with increasing curvature. For zigzag SWNTs, the energy of this singlet $\pi^{*}$ state is shifted downward with increasing curvature.

In figure 3(a), the doubly degenerate $\pi$ states (which are the valence band edge at the $\Gamma$ point), the doubly degenerate $\pi^{*}$ states (which become the conduction band edge at $\Gamma$ for large $R$ ) and the singlet $\pi^{*}$ state (which is in the conduction band for large $R$ ) are shown. As seen, the shift of the singlet $\pi^{*}$ state is curvature dependent, and below a certain radius determines the band gap. For tubes with radius greater than $3.3 \AA$ (i.e. $n>8$ ), the energy of the singlet $\pi^{*}$ state at the $\Gamma$ point of the BZ is above the doubly degenerate $\pi^{*}$ states (i.e. the bottom of the 
conduction band), while it falls between the valence and conduction band edges for $n=7,8$, and eventually dips even below the doubly degenerate valence band $\pi$ states for the zigzag SWNT with radius less than $2.7 \AA$ (i.e. $n<7$ ). Therefore, all the zigzag tubes with radius less than $2.7 \AA$ are metallic. For $n=7,8$, the edge of the conduction band is set by the singlet $\pi^{*}$ state, but not by the doubly degenerate $\pi^{*}$ state. The band gap derived from the zone folding scheme is reduced by the shift of this singlet $\pi^{*}$ state as a result of curvature induced $\sigma^{*}-\pi^{*}$ mixing. This explains why the tight binding calculations predict band gaps around $1 \mathrm{eV}$ for $n=7,8$ tubes, while the self-consistent calculations predict much smaller values. Based on $\pi$ orbital tight binding model, it was proposed [13] that $E_{\mathrm{g}}$ behaves as

$$
E_{\mathrm{g}}=\gamma_{0} \frac{d_{0}}{R}
$$

which is independent of the helicity. Within the simple $\pi$ orbital tight binding model, $\gamma_{0}$ is taken to be equal to the hopping matrix element $V_{\mathrm{pp} \pi} . d_{0}$ is the bond length in graphene. However, as seen in figure 3(b), the band gap displays a rather oscillatory behaviour. The relation given in equation (1) was obtained by a second-order Taylor expansion of one-electron eigenvalues of the $\pi$ orbital tight binding model [13] around the K point of the BZ, and hence it fails to represent the effect of the helicity. By extending the Taylor expansion to the next higher order, Yorikawa and Muramatsu [41, 42] included another term in the empirical expression of the band gap variation,

$$
E_{\mathrm{g}}=V_{\mathrm{pp} \pi} \frac{d_{0}}{R}\left[1+(-1)^{p} \gamma \cos (3 \theta) \frac{d_{0}}{R}\right],
$$

which depends on the chiral angle, $\theta$, as well as an index $p$. Here $\gamma$ is a constant and the index $p$ is defined as the integer from $k=n-2 m=3 q+p$. The factor $(-1)^{p}$ comes from the fact that the allowed $\mathbf{k}$ is nearest to either the $\mathrm{K}$ or $\mathrm{K}^{\prime}$ point of the hexagonal Brillouin zone. For zigzag nanotubes $\theta=0$, and hence the solid curves in figure 3(b) are fits to the empirical expression $E_{\mathrm{g}}=V_{\mathrm{pp} \pi} d_{0} / R \pm V_{\mathrm{pp} \pi} \gamma d_{0}^{2} / R^{2}$, obtained from equation (2) by using the parameters $V_{\mathrm{pp} \pi}=2.53 \mathrm{eV}$ and $\gamma=0.43$. The agreement between the first-principles calculations [18] and the STS data [29, 30] is very good considering the fact that there might be some uncertainties in identifying the nanotube in the experiment.

The situation displayed in figure 3 indicates that the variation of the band gap with the radius is not simply $1 / R$, but additional terms incorporating the chirality dependence are required. Most importantly, the mixing of the singlet $\pi^{*}$ state with the singlet $\sigma^{*}$ state due to the curvature and its shift towards the valence band with increasing curvature are not included in either the $\pi$ orbital tight binding model or the empirical relations expressed by equations (1) and (2). This behaviour of the singlet $\pi^{*}$ states is of particular importance for the applied radial deformation that modifies the curvature and in turn induces metallization [24, 43, 44] as discussed in sections 6.2 and 7.2.

\section{Hydrogenation of carbon nanotubes}

Owing to their large effective surface area, carbon nanotubes can be suitable for hydrogen storage. Several studies have explored this capacity of carbon nanotubes [45-51], but the results reported to date have been conflicting. Diverse hydrogen storage densities ranging from 0.4 to $10 \mathrm{wt} \%$ have been reported. Theories based on physisorption have failed to predict high hydrogen uptake [52]. Recently, hydrogen adsorption has been the subject of various studies predicting interesting features [53-55, 57, 58]. Tada et al [54] examined the dissociative adsorption of a hydrogen molecule on various SWNTs. They found that the potential barrier height for the dissociative adsorption of a hydrogen molecule onto an outer wall decreases as 
the tube diameter decreases. Andriotis et al [58] calculated the $I-V$ characteristics of finite $(5,5)$ and $(10,0)$ SWNTs by using the Landauer expression [59] within the tight binding Green function method. They found extreme sensitivity to hydrogen adsorption. On the basis of first-principles calculations, Chan et al [60] proposed a mechanism for the dissociative chemisorption of hydrogen molecules on SWNTs in the solid (rope) phase under external pressure. They argued that the process is reversible upon the release of external pressure.

Extensive analysis of hydrogen chemisorption on SWNTs has been carried out by Yildirim et al [55] using the first-principles pseudopotential plane wave method. They calculated the average binding energy $\overline{E_{\mathrm{b}}}=\left(E_{\mathrm{T}}[\mathrm{SWNT}]+4 n E_{\mathrm{T}}[\mathrm{H}]-E_{\mathrm{T}}[\mathrm{H}+\mathrm{SWNT}]\right) / 4 n$, in terms of the total energies of a fully relaxed bare SWNT, the single hydrogen atom $\mathrm{H}$ and a fully exohydrogenated SWNT, respectively. They found that $\overline{E_{\mathrm{b}}}$ increases with decreasing radius of the exohydrogenated SWNT, $R_{\mathrm{HC}}$, monotonically. The binding energies can be very well described by a one-parameter fit, $\overline{E_{\mathrm{b}}}=E_{0}+C(n, m) / R_{\mathrm{HC}}$. Here $E_{0}=1.73 \mathrm{eV}$; $C(n, n)=4.45 \mathrm{eV}$ and $C(n, 0)=4.62 \mathrm{eV}$. Interestingly, a dodecahedron and $\mathrm{C}_{60} \mathrm{H}_{60}$ have higher average binding energies for adsorbed hydrogen atom. Also the binding energies of zigzag tubes are always lower than those of armchair tubes with equal radius by about $30 \mathrm{meV} /$ atom.

Although the exohydrogenated SWNT $\left(\mathrm{C}_{4 n} \mathrm{H}_{4 n}\right)$ is always energetically favourable with respect to a bare SWNT and $4 n \mathrm{H}$ atoms for all values of the radius, it is of interest to see whether they are also stable against breaking a single $\mathrm{C}-\mathrm{H}$ bond (or desorption of a single $\mathrm{H}$ atom). Yildirim et al [55] revealed that for $R_{\mathrm{HC}}>6.25 \AA$ the required energy becomes negative and hence the system becomes unstable, in spite of the fact that there might be an energy barrier preventing the desorption of $\mathrm{H}$ atoms. On the other hand, the adsorption of a single $\mathrm{H}$ atom to a bare SWNT is always exothermic no matter what the value of $R$ is. However, as discussed extensively in section 6.3 the amount of energy gained by the adsorption of a single $\mathrm{H}$ atom decreases as $R$ increases.

Important effects of hydrogenation have been further examined for different isomers at different hydrogen coverage [57]. At full coverage, these are full exohydrogenation and endoexohydrogenation where each carbon atom is bonded to a hydrogen alternately from inside and outside. For half-coverage, i.e. $\Theta=0.5$, denoted by $\mathrm{C}_{4 n} \mathrm{H}_{2 n}$, there are three isomers, namely (i) a uniform pattern, where every other carbon atom is bonded to a hydrogen atom from outside, (ii) a chain pattern, where every other carbon zigzag chain is saturated by hydrogen, and (iii) a dimer pattern where every other carbon dimer row perpendicular to the zigzag carbon chains is saturated by hydrogen as described in figure 4(a).

It is found that geometric and electronic structures and binding energies of hydrogenated SWNTs strongly depend on the pattern of hydrogenation (i.e. decoration). The most remarkable effect is obtained when zigzag nanotubes are uniformly exohydrogenated at $\Theta=0.5$. Upon hydrogenation the structure undergoes a massive reconstruction, whereby the circular cross section of the $(7,0)$ SWNT changes to a rectangular one, and those of $(8,0)$, $(9,0),(10,0)$ and $(12,0)$ change to square ones as shown in figure 4(b). These new structures are stabilized by the formation of new diamond-like $\mathrm{C}-\mathrm{C}$ bonds with $d_{\mathrm{C}-\mathrm{C}} \sim 1.51-1.63 \AA$ near the corners of rectangular or square $\mathrm{C}_{4 n} \mathrm{H}_{2 n}$. Hence, triangular and pentagonal $\mathrm{C}$ rings are formed instead of hexagonal ones. Depending on $2 n \bmod 4$, either one bond is formed just at the corners or two bonds are formed at either side of the corners. Most interestingly, all these structures are predicted to be metallic with a high density of states at the Fermi level [57]. The uniform adsorption at $\Theta=0.5$ is metastable for zigzag nanotubes. Such a local minimum does not exist for armchair nanotubes, since uniformly adsorbed $\mathrm{H}$ atoms are rearranged upon relaxation by concerted exchange of $\mathrm{C}-\mathrm{H}$ bonds to form zigzag chains along the tube axis. The cross sections of the chain isomer at $\Theta=0.5$ for armchair tubes are polygonal where 

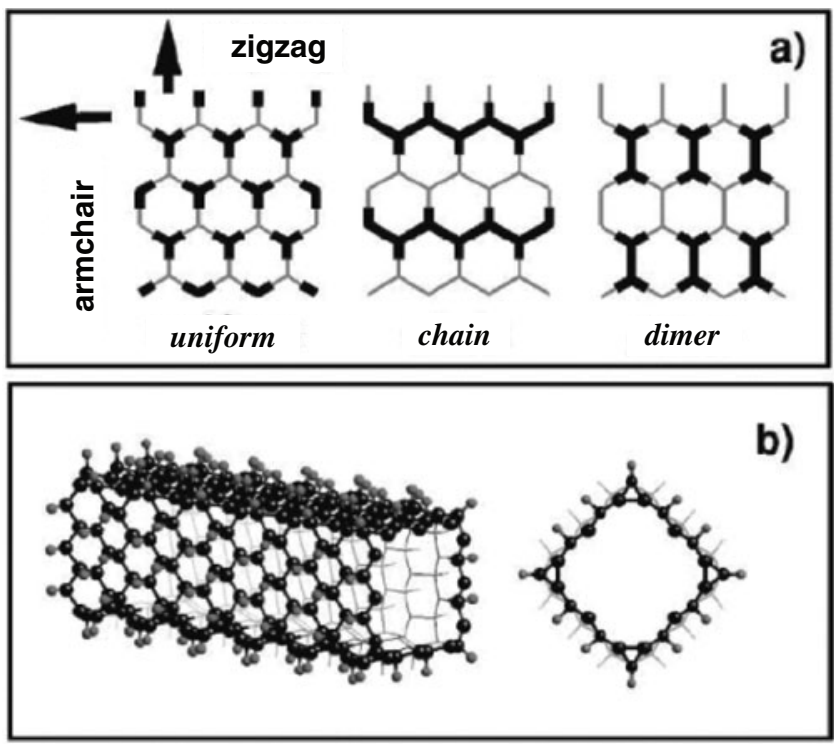

Figure 4. (a) A view of three different isomers of hydrogenated $(n, 0)$ SWNT at half-coverage (with formula unit $\mathrm{C}_{4 n} \mathrm{H}_{2 n}$ ). The left and up arrows indicate the tube axis for armchair and zigzag nanotubes, respectively. Carbon atoms which are bonded to hydrogens are indicated by dark colour. (b) A side and top view of a $(12,0) \mathrm{C}_{48} \mathrm{H}_{24}$ showing the square cross section of a uniformly exohydrogenated nanotube at half-coverage. (Reproduced from [57].)

the corners are pinned by the zigzag $\mathrm{H}$ chains along the tube axis. For other isomers at halfcoverage as well as exohydrogenations and endo-exohydrogenations at full coverage, the cross sections remain quasi-circular (see figure 5).

The average binding energies are plotted as a function of $R$ in figure 5, and fitted to a general expression, $\overline{E_{\mathrm{b}}}=E_{0}(\Theta)+\frac{C_{p}(\Theta)}{R^{p}}$. A special case of this fit was employed above for exohydrogenated tubes using $R_{\mathrm{HC}}$ (i.e. expanded radius upon hydrogenation). Note that the $1 / R$ form (i.e. $p=1$ ) is quite common for SWNTs and scales various properties $[16,18,26,27,55,61]$. Here $C_{p}(\Theta)$ is a constant that depends on coverage $\Theta$, and represents the curvature effect. The values of the exponent $p$ and $C_{p}$ are determined for different isomers [57]. We note that while $\overline{E_{\mathrm{b}}}$ increases with decreasing $R$ in the case of exohydrogenation, this trend is reversed for endo-exohydrogenation due to increased $\mathrm{H}-\mathrm{H}$ repulsion inside the tube at small $R$. Nevertheless, the endo-exohydrogenation of SWNTs, which transforms the $\mathrm{sp}^{2}$ to $\mathrm{sp}^{3}$-like bonding, gives rise to the highest binding energy saturating at $3.51 \mathrm{eV}$ as $R \rightarrow \infty$. At this limit, the exo-endohydrogenated graphene (from above and below) is buckled by $0.46 \AA$ as if two diamond (111) planes had an interplanar distance of $0.50 \AA$.

The effect of exohydrogenation on the electronic structure is remarkable. The band gaps of $(n, 0)$ zigzag tubes have increased approximately by $2 \mathrm{eV}$ upon full exohydrogenation. Even more interesting is that the exohydrogenated metallic armchair tube becomes semiconducting with a band gap larger than the zigzag one for comparable radius. As for the band gap variation with $R, E_{\mathrm{g}}(R)$ for exohydrogenated tubes at $\Theta=1$ displays a behaviour similar to that of $\bar{E}_{\mathrm{b}}(R)$ for both types of SWNTs and hence decreases with increasing $R$ (see figure 6). Relatively larger band gaps (in the range of 3.5-4 eV) of endo-exohydrogenated SWNTs $(\Theta=1)$ can be explained by the fact that the adsorption of $\mathrm{H}$ alternately inside and outside 


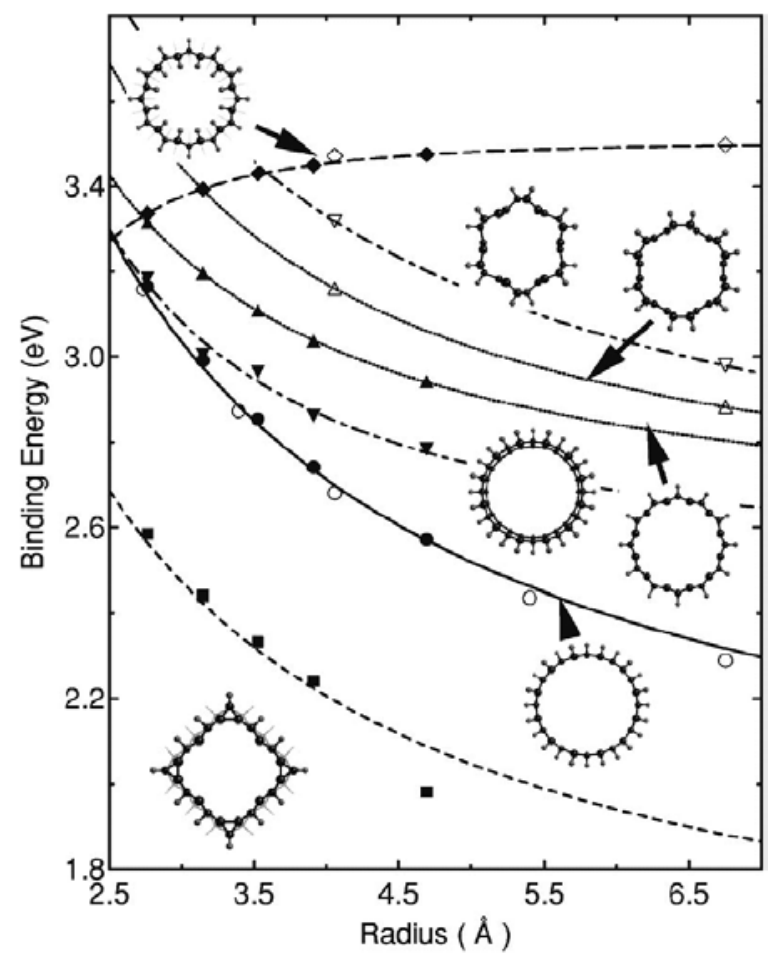

Figure 5. Average binding energies, $\overline{E_{\mathrm{b}}}$, of hydrogen atoms adsorbed on various zigzag and armchair SWNTs versus bare tube radius $R$. Filled and open symbols are for zigzag and armchair nanotubes, respectively. Circles and diamonds are for exohydrogenation and endoexohydrogenation at full coverage, respectively. The filled squares show the zigzag nanotubes uniformly exohydrogenated at half coverage. The chain and dimer patterns of adsorbed hydrogen atoms at half coverage are shown by down and up triangles, respectively. Curves are analytical fits explained in the text. Insets show top views of several $\mathrm{C}_{4 n} \mathrm{H}_{2 n}$ isomers. (Reproduced from [57].)

leads to an atomic configuration closer to the diamond structure having a rather large band gap $\left(E_{\mathrm{g}}=5.4 \mathrm{eV}\right)$.

The effect of hydrogenation on the electronic structure is even more interesting at $\Theta=0.5$. Depending on the pattern of hydrogen adsorption, an isomer can be either a metal or insulator. For example, all uniform $\mathrm{C}_{4 n} \mathrm{H}_{2 n}$ are metallic. On the other hand, the chain pattern realized on the $(n, 0)$ SWNTs results in two doubly degenerate, almost dispersionless states at the valence and conduction band edges. The band gap $E_{\mathrm{g}}$ between these states decreases with increasing $R$. When $n$ is odd, $E_{\mathrm{g}}$ is large (e.g. $E_{\mathrm{g}}=2.1 \mathrm{eV}$ for $(7,0)$ ). When $n$ is even, the doubly degenerate band at the conduction band edge moves towards the valence band edge and splits into bonding and antibonding states. As a result $E_{\mathrm{g}}$ is reduced significantly, becoming only a pseudogap for large and even $n$ values. Finally, the dimer row isomers are insulators and $E_{\mathrm{g}}$ increases with increasing radius. Surprisingly, there are two dispersive bands with $\sim 1 \mathrm{eV}$ bandwidth at both band edges and the extremum moves from the centre of the Brillouin zone ( $\Gamma$ point) to the zone edge $(\mathrm{Z}$ point).

Hydrogen adsorption induced dramatic changes of the electronic structure are demonstrated by the total density of states (DOS), $\mathcal{D}(E)$, of $(9,0)$ nanotubes in figure 7 . First of all, the small band gap of the bare $(9,0)$ SWNT is opened by $\sim 2 \mathrm{eV}$ upon exohydrogenation at $\Theta=1$. The band gap is still significant for $\Theta=0.5$, having the chain pattern, and increases 


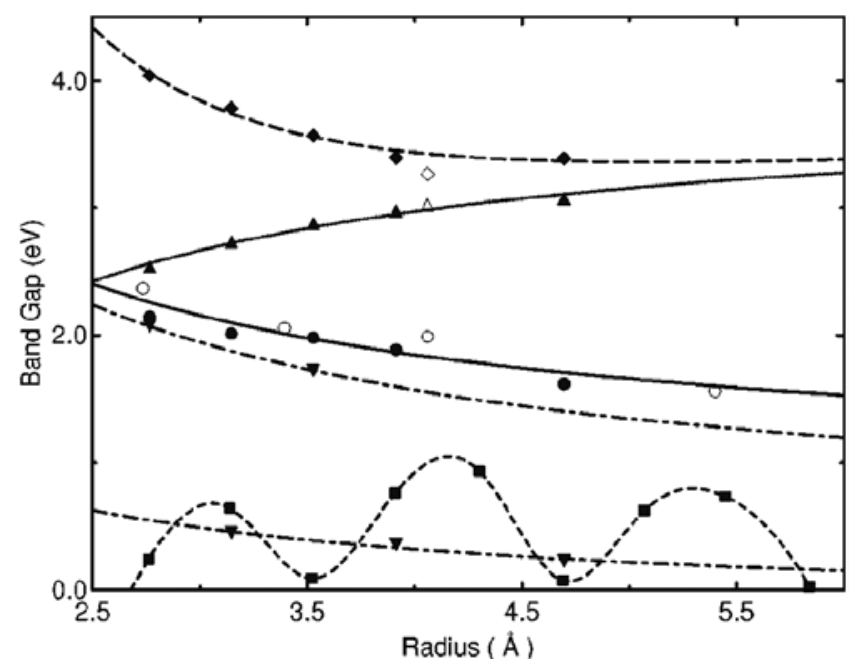

Figure 6. The band gaps, $E_{\mathrm{g}}$, versus the bare nanotube radius $R$. Filled and empty symbols indicate zigzag and armchair SWNTs, respectively. Squares show nonmonotonic variation of the band gap of the bare zigzag nanotubes. Exohydrogenated and endo-exohydrogenated nanotubes $(\Theta=1)$ and chain and row patterns of adsorbed hydrogen atoms at half-coverage are shown by circles, diamonds, down and up triangles, respectively. Curves are guides to the eye. (Reproduced from [57].)

to $4 \mathrm{eV}$ for the dimer pattern. However, a similar chain pattern of $\mathrm{C}_{32} \mathrm{H}_{16}$ in figure 7 has a much smaller band gap. Surprisingly, all zigzag nanotubes uniformly exohydrogenated at $\Theta=0.5$ are metals. As displayed in the sixth panel of figure 7 for $\mathrm{C}_{36} \mathrm{H}_{18}$ (uniform), their total densities of states are characterized by a peak yielding a high state density at $E_{\mathrm{F}}$. While carbon states are pushed apart, yielding a $\sim 4-5 \mathrm{eV}$ gap, a new dispersive metallic band with $\sim 1-2 \mathrm{eV}$ bandwidth crosses the Fermi level. Apart from this being an ideal 1D conductor, the very high density of states at $E_{\mathrm{F}}$ might lead to superconductivity. Note that these uniform $\mathrm{C}_{4 n} \mathrm{H}_{2 n}$ undergo a massive reconstruction and their circular cross sections change into square ones with the formation of new $\mathrm{C}-\mathrm{C}$ bonds at the corners. All $\mathrm{C}$ atoms without attached $\mathrm{H}$ (except those at corners) as well as the $\mathrm{H}$ atoms at the centre of four planar sides contribute to the high $\mathcal{D}\left(E_{\mathrm{F}}\right)$. In this way four individual conduction paths are formed on each side of a square tube. It is emphasized that the transformation from the $\mathrm{sp}^{2}$ to the $\mathrm{sp}^{3}$ bonding underlies the above effects. For $\mathrm{C}_{4 n} \mathrm{H}_{2 n}$, especially endo-exohydrogenated SWNTs, $\mathrm{C}_{4 n} \mathrm{H}_{2 n} \mathrm{H}_{2 n}$, the structures can be conceived as if they are more diamond-like than graphitic. This argument is justified by the comparison of $\mathcal{D}(E)$ for the endo-exohydrogenated $(9,0)$, i.e. $\mathrm{C}_{36} \mathrm{H}_{18} \mathrm{H}_{18}$, with that of bulk diamond in figure 7. Apart from opening a large band gap, the quasi-metallic $\mathcal{D}(E)$ of the bare $(9,0)$ is modified to become similar to that of bulk diamond. The latter has a relatively larger valence bandwidth due to the coupling of distant neighbours.

\section{Oxygenation of carbon nanotubes}

Remarkable effects on the electrical resistance of a semiconducting single-wall carbon nanotube (s-SWNT) upon exposure to gaseous molecules such as $\mathrm{NO}_{2}$ and $\mathrm{NH}_{3}$, have been reported [62]. Collins et al [63] found similar effects for oxygen. Exposure to air or oxygen influences electrical resistance and the thermoelectric power of a s-SWNT, which can be converted to a good metal and hence its electronic properties can be reversibly modified by a 


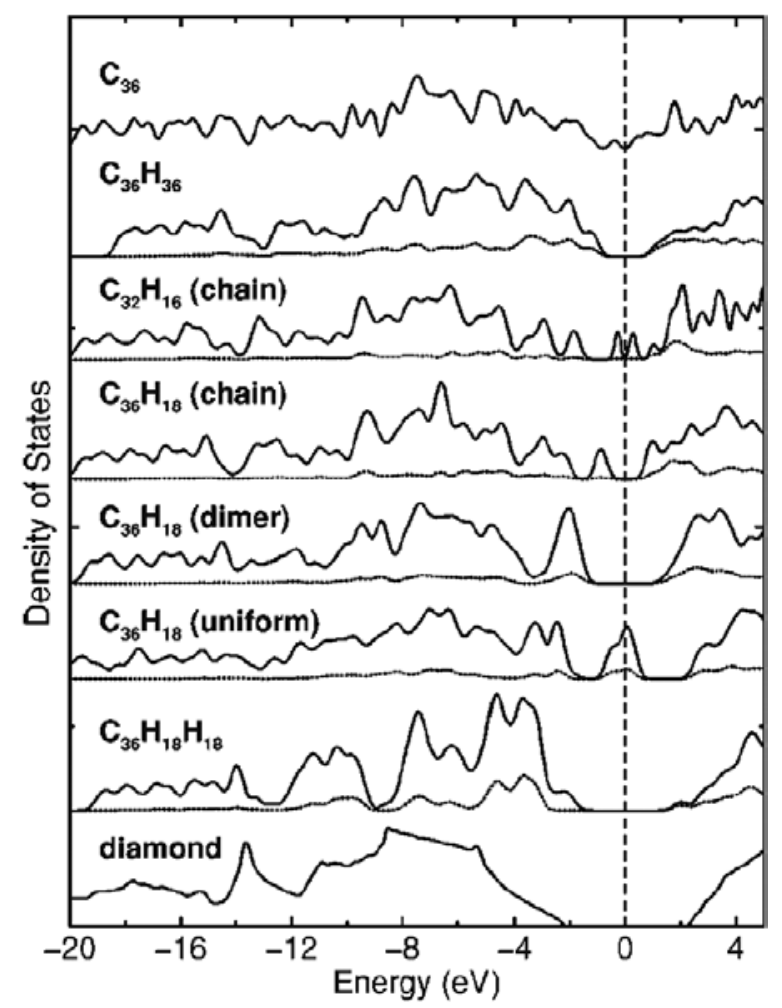

Figure 7. Comparison of the electronic density of states (DOS) of a bare $(9,0)$ nanotube $\left(\mathrm{C}_{36}\right)$ and its various hydrogenated isomers. The DOS of hydrogenated $(8,0)$ at $\Theta=0.5$ (i.e. $\mathrm{C}_{32} \mathrm{H}_{16}$ in a chain pattern) and bulk diamond (bottom panel) are also shown for comparison. The zero of energy is taken at the Fermi energy shown by a vertical dashed line. (Reproduced from [57].)

surprisingly small concentration of adsorbed oxygen. Experimental studies on carbon nanotube field emitters have shown that the adsorption of ambient gases, in particular $\mathrm{O}_{2}$, instantaneously induces a significant increase in the emission current [64]. In addition to functionalization, oxygenation is involved in other applications. For example, carbon nanotubes synthesized by using arc discharge are purified from other undesired, carbon based nanoparticles through oxidation. At elevated temperatures, oxygen undergoes chemical reaction preferably with the strained $\mathrm{C}-\mathrm{C}$ bonds and eliminates carbonaceous nanoparticles as well as the caps of nanotubes [65-67].

\subsection{Physisorption of $\mathrm{O}_{2}$ molecules}

Observed effects on the electronic structure of SWNTs due to $\mathrm{O}_{2}$ physisorption have been subject to recent theoretical investigations based on first-principles calculations [68-77]. Spin unpolarized band structure calculations based on the local density approximation (LDA) predicted that the semiconducting $(8,0)$ tube becomes metallic, since the valence band is hole doped by the Fermi level touching the top of the valence band as a result of $\mathrm{O}_{2}$ physisorption [68]. The analysis based on the local spin density approximation (LSDA) has indicated that the physisorbed $\mathrm{O}_{2}$ favours the triplet state. While the spin up states are fully occupied, the spin down states are nearly empty and hence give rise to a finite density of states at the Fermi level [68]. 


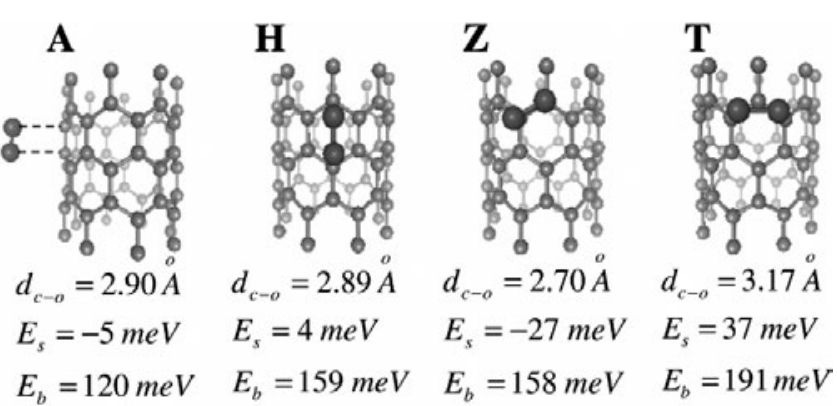

Figure 8. A schematic illustration of the physisorption sites of $\mathrm{O}_{2}$ molecules on the $(8,0) \mathrm{SWNT}$. The GGA optimized distance from one $\mathrm{O}$ atom of the molecule to the nearest $\mathrm{C}$ atom of the SWNT is denoted by $d_{\mathrm{C}-\mathrm{O}} . E_{\mathrm{S}}$ is the GGA chemical bonding energy for the spin polarized triplet state. $E_{\mathrm{b}}$ is the binding energy including the van der Waals interaction. (Reproduced from [75].)

The energetics of oxygen adsorption on the surface of graphite [78] and on an (8, 0) SWNT were calculated for selected sites [69]. Adsorption and desorption of an oxygen molecule and various precursor states at the edges of finite size armchair $(5,5)$ and zigzag $(9,0)$ SWNTs were studied to provide an understanding of the oxidative etching process [70]. Similarly, the mechanism of the oxidative etching of the caps and walls of the small radius $(5,5)$ armchair SWNT was investigated [71]. The effects of oxygen adsorption on the field emission from carbon nanotubes were treated by using an ab initio approach [72]. The dynamics of the thermal collision of the $\mathrm{O}$ atom with a $(6,0)$ SWNT was simulated by ab initio calculations [73].

A detailed analysis of the physisorption of oxygen molecules based on first principles and fully relaxed, spin polarized GGA calculations including the long range interactions and the chemisorption of oxygen atom on the $(8,0)$ and $(6,6)$ SWNTs has been carried out by Dag et al $[75,77]$ with the aim of revealing how the adsorptions of $\mathrm{O}_{2}$ and $\mathrm{O}$ modify the electronic properties. The zigzag $(8,0)$ and armchair $(6,6)$ tubes are taken as prototypes for the s-SWNT and metallic m-SWNT, respectively. Similar trends are expected to occur in other tubes with curvature effects being emphasized at small $R$ [18]. The bonding of $\mathrm{O}_{2}$ has been studied by placing the molecule at different sites on the SWNT as shown in figure 8 .

The binding energy involves short range chemical interaction and long range van der Waals interaction, i.e. $E_{\mathrm{b}}=E_{\mathrm{s}}+E_{\mathrm{vdW}}$. Dag et al [75] have calculated the contribution of the short range interaction (i.e. chemical bonding energy) by using the expression

$$
E_{\mathrm{s}}=E_{\mathrm{T}}[\mathrm{SWNT}]+E_{\mathrm{T}}\left[\mathrm{O}_{2}\right]-E_{\mathrm{T}}\left[\mathrm{O}_{2}+\mathrm{SWNT}\right],
$$

in terms of the GGA spin polarized total energies of the fully optimized bare SWNT $\left(E_{\mathrm{T}}[\mathrm{SWNT}]\right)$, the molecule $\left(E_{\mathrm{T}}\left[\mathrm{O}_{2}\right]\right)$ and $\mathrm{O}_{2}$ physisorbed on the $\mathrm{SWNT}\left(E_{\mathrm{T}}\left[\mathrm{O}_{2}+\mathrm{SWNT}\right]\right)$. By definition, $E_{\mathrm{s}}>0$ in equation (3) corresponds to a stable and exothermic chemical bonding. In some cases, $E_{\mathrm{s}}<0$ is endothermic, but it corresponds to a local minimum on the BornOppenheimer surface, where the desorption of $\mathrm{O}_{2}$ from the SWNT is prevented by a barrier. The spin polarized calculations yield relatively lower (stronger) total energies, $E_{\mathrm{T}}\left[\mathrm{O}_{2}+\mathrm{SWNT}\right]$, and hence set the triplet state as the ground state with a net magnetic moment of $\sim 2 \mu_{\mathrm{B}}$ ( $\mu_{\mathrm{B}}=$ Bohr magneton) per unit cell. The corresponding values for $E_{\mathrm{s}}$ are $-5,4,-27$ and $37 \mathrm{meV}$, for $\mathrm{A}, \mathrm{H}, \mathrm{Z}$ and $\mathrm{T}$ sites (see figure 8).

In the case of the physisorption, the contributions of short range and long range interactions are small and comparable. Therefore, in treating the binding energy of the physisorbed $\mathrm{O}_{2}$ the attractive vdW energy is calculated by using the asymptotic form of the Lifshitz formula [7981], i.e. $E_{\mathrm{vdW}}=\sum_{i j} C_{6 i j} / r_{i j}^{6}$. A positive sign has been assigned to $E_{\mathrm{vdW}}$, since stable binding 
is specified by positive energies. Here $r_{i j}$ is the distance between the $i$ th $\mathrm{O}$ atom and the $j$ th $\mathrm{C}$ atom, and the constant $C_{6 i j}$ is calculated within the Slater-Kirkwood approximation [82] to be $10.604 \mathrm{eV} \AA^{6}$. We note, however, that this standard calculation of vdW energy may involve ambiguities due to the relatively small distance between the physisorbed molecule and the SWNT. Nevertheless, the vdW interaction is attractive and strengthens the chemical bond. Using the binding geometries determined from the minimization of GGA total energies, the $E_{\mathrm{vdW}}$ values are calculated to be $125,155,185$ and $155 \mathrm{meV}$ for $\mathrm{A}, \mathrm{H}, \mathrm{Z}$ and T sites, respectively [75]. By adding these $E_{\mathrm{vdW}}$ values to the corresponding GGA chemical bonding energies $E_{\mathrm{s}}$, the binding energies $E_{\mathrm{b}}$ of physisorbed $\mathrm{O}_{2}$ are found to be $120,159,158$ and $191 \mathrm{meV}$ for A, H, Z and T sites, respectively (see figure 8). The binding energy of the $\mathrm{T}$ site is in agreement with the recent measurement by Ulbricht et al [83]. We note that these binding energies are small, and become exothermic mainly owing to the long range vdW interaction. The small binding energies are characteristic of physisorption.

Using a similar first-principles method within the local density approximation (LDA), Jhi et al [68] found $E_{\mathrm{s}}=250 \mathrm{meV}$ for the A site physisorption. The relatively large value of $E_{\mathrm{s}}$ is due to the fact that the LDA yields overbinding as compared to the GGA. The physisorption of $\mathrm{O}_{2}$ on the $(6,6)$ armchair SWNT has been studied for two possible physisorption sites; i.e. above the centre of the hexagon ( $\mathrm{H}$ site), and on top of the $\mathrm{C}-\mathrm{C}$ bond and perpendicular to the axis of the tube (B site) [75]. The B site of a $(6,6)$ tube is similar to the A site of a $(8,0)$ tube, but in the former case the $\mathrm{C}-\mathrm{C}$ bond under the adsorbed $\mathrm{O}_{2}$ is highly strained, since it lies on the circumference. The binding energies are calculated to be 132 and $106 \mathrm{meV}$ for $\mathrm{H}$ and $\mathrm{B}$ sites, respectively.

While the spin polarized LDA (LSDA) energy band structure [68] has been interpreted as hole doping due to the adsorbed oxygen molecule, Derycke et al [84] have argued that the main effect of oxygen physisorption is not to dope the bulk of the tube, but to modify the barriers of the metal- $(8,0)$ SWNT contact. The hole doping picture has been refuted by recent first-principles calculations [75-77]. The band structure calculated using the spin polarized GGA for $\mathrm{O}_{2}$ physisorbed at the A site has yielded a band gap of $0.2 \mathrm{eV}$ between the top of the valence band of the $(8,0)$ tube and the empty $\mathrm{O}_{\mathrm{pp} \pi^{*}}(\downarrow)$ band (see figure 9 ). Band structures corresponding to $\mathrm{H}, \mathrm{Z}$ and $\mathrm{T}$ sites also have band gaps, which are large enough to prevent thermal excitation of electrons from the valence band at room temperature [77].

The situation for the m-SWNT is quite different. Upon $\mathrm{O}_{2}$ physisorption the metallicity of the armchair tube is lifted for the spin down bands, while spin up bands continue to cross at the Fermi level, and make the system metallic only for one type of spin. The splitting of the bands can be explained by the broken symmetry [85].

The interaction of $\mathrm{O}_{2}$ with the SWNT as a function of $\mathrm{O}_{2}-\mathrm{SWNT}$ distance $d$ has been examined by calculating the total energy $E_{\mathrm{T}}$, bond distance of $\mathrm{O}_{2} d_{\mathrm{O}-\mathrm{O}}$, magnetic moment $\mu$ and energy gap $E_{\mathrm{g}}$ of the $\mathrm{O}_{2}$ physisorbed $(8,0) \mathrm{SWNT}$ [77]. In these calculations, $d$ has been constrained, but $d_{\mathrm{O}-\mathrm{O}}$ has been relaxed. Figure 10(a) shows the variation of the ratios of $E_{\mathrm{T}}$, $d_{\mathrm{O}-\mathrm{O}}$, magnetic moment $\mu$ and $E_{\mathrm{g}}$ to their corresponding equilibrium values at $d^{0}=2.89 \AA$. We see that $\left|E_{\mathrm{T}}\right|$ decreases in the range $1.6 \AA<d<2.9 \AA$. For a wide range of $\mathrm{O}_{2}-\mathrm{SWNT}$ separation, $E_{\mathrm{g}}$ continues to exist, and the total energy difference between the spin polarized and spin unpolarized states (i.e. $\Delta E_{\mathrm{T}}=0.86 \mathrm{eV}$ ) induces the gap $E_{\mathrm{g}}$ and prevents it from closing. For $\sim 1.6 \AA<d<d^{0}$ a strong perpendicular force $F_{\perp}$ is generated on the $\mathrm{O}_{2}$ molecule to push it away from the SWNT. The magnetic moment of $\mathrm{O}_{2}+\mathrm{SWNT}$ diminishes at a distance $d<2 \AA$. Moreover, the singlet state of adsorbed $\mathrm{O}_{2}$ leads to a bound state at the A site at $d=1.47 \AA$, with an energy $0.45 \mathrm{eV}$ above the corresponding physisorption state (i.e. $E_{\mathrm{T}}-E_{\mathrm{T}}^{0}=0.45 \mathrm{eV}$ ). A similar singlet bound state occurs also at the $\mathrm{Z}$ site at 


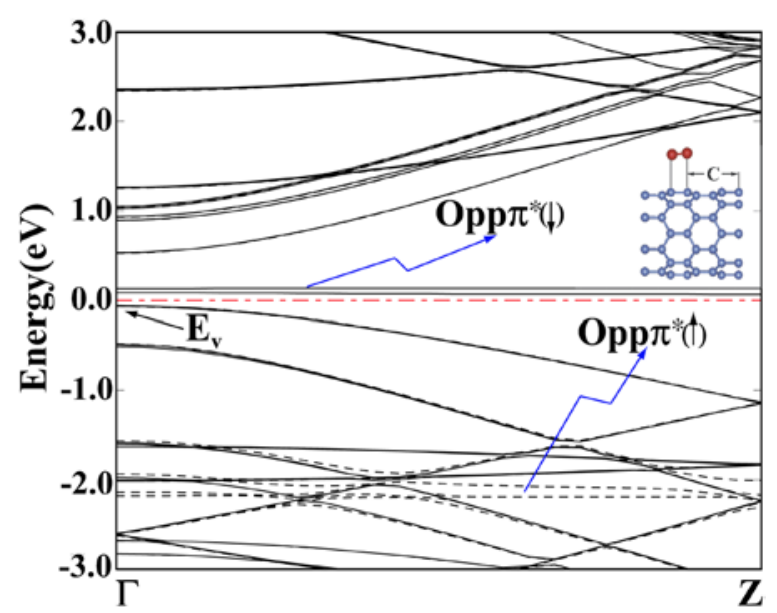

Figure 9. The electronic structure of $\mathrm{O}_{2}$ physisorbed on an $(8,0) \mathrm{SWNT}$ calculated using the spin polarized GGA with the atomic structure fully optimized in the double cell. The spin up and spin down bands corresponding to the triplet ground state are shown by broken and continuous lines, respectively. The zero of energy is set at the Fermi level. $E_{\mathrm{V}}$ indicates the top of the valence band. The adsorption site (A site) is shown in the inset. (Reproduced from [75].)

$d=1.47 \AA$ and $0.80 \mathrm{eV}$ above the physisorption state of the $\mathrm{Z}$ site at $d=2.70 \AA$. These singlet bound states in figure 10 (b) correspond to local minima on the Born-Oppenheimer surface and are separated from the more energetic physisorption states by an energy barrier. It is noted that these states $[77,86]$ are neither easily accessible from the physisorption state, nor support the hole doping picture because the band gap is $\sim 0.5 \mathrm{eV}$. However, $\mathrm{O}_{2}$ adsorbed at the $\mathrm{H}$ site of the $(6,6)$ SWNT behave differently. Figures 10(c) and (d) show the variation of $E_{\mathrm{T}}(d), F_{\perp}(d), \mu(d)$ and $d_{\mathrm{O}-\mathrm{O}}(d)$ where at $d \sim 1.25 \AA, d_{\mathrm{O}-\mathrm{O}}$ is increased to $2.5 \AA$ and hence the bond is broken, i.e. the $\mathrm{O}_{2}$ molecule dissociates into two $\mathrm{O}$ atoms. In contrast to the case at this site, the $\mathrm{O}_{2}$ molecule has a bound singlet state at the $\mathrm{B}$ site with $d_{\mathrm{O}-\mathrm{O}}=1.51 \AA$.

\subsection{Chemisorption of oxygen atoms}

The effect of the adsorbed oxygen on the structural and electronic properties of a SWNT is important. Breaking of the $\mathrm{O}-\mathrm{O}$ bond of a physisorbed $\mathrm{O}_{2}$ molecule is unlikely owing to the weak interaction with the SWNT. However, it was shown that near the defect sites of the graphite surface $\mathrm{O}_{2}$ molecules can dissociate [78]. A carbon nanotube, that can be described as graphene rolled into a cylinder, is normally more reactive than the surface of graphite. As a result, $\mathrm{O}_{2}$ physisorbed near the defect sites of a SWNT is expected to dissociate into atomic oxygens. In fact, it was shown that there is no activation barrier for the dissociation of $\mathrm{O}_{2}$ when it is adsorbed at the zigzag edge of a SWNT [70]. Owing to the concerted motion of the atoms at the proximity of the molecule and energy gained by the individual oxygen atoms engaging in the bonding with the SWNT concomitant with the dissociation, the activation energy for dissociation is expected to be low. In this section, we discuss the interaction between atomic $\mathrm{O}$ and SWNT, and reveal the nature of the chemical bonding. Only the short range interaction is considered.

Spin polarized calculations yield the singlet state with a net zero magnetic moment as the ground state. Various adsorption sites together with the geometry and chemisorption energies $E_{\mathrm{s}}$ obtained from spin unpolarized calculations are shown in figure 11. Among all sites considered in [75], the single $\mathrm{O}$ adsorbed on top of the zigzag $\mathrm{C}-\mathrm{C}$ bond (i.e. the $\mathbf{z}$ site) 

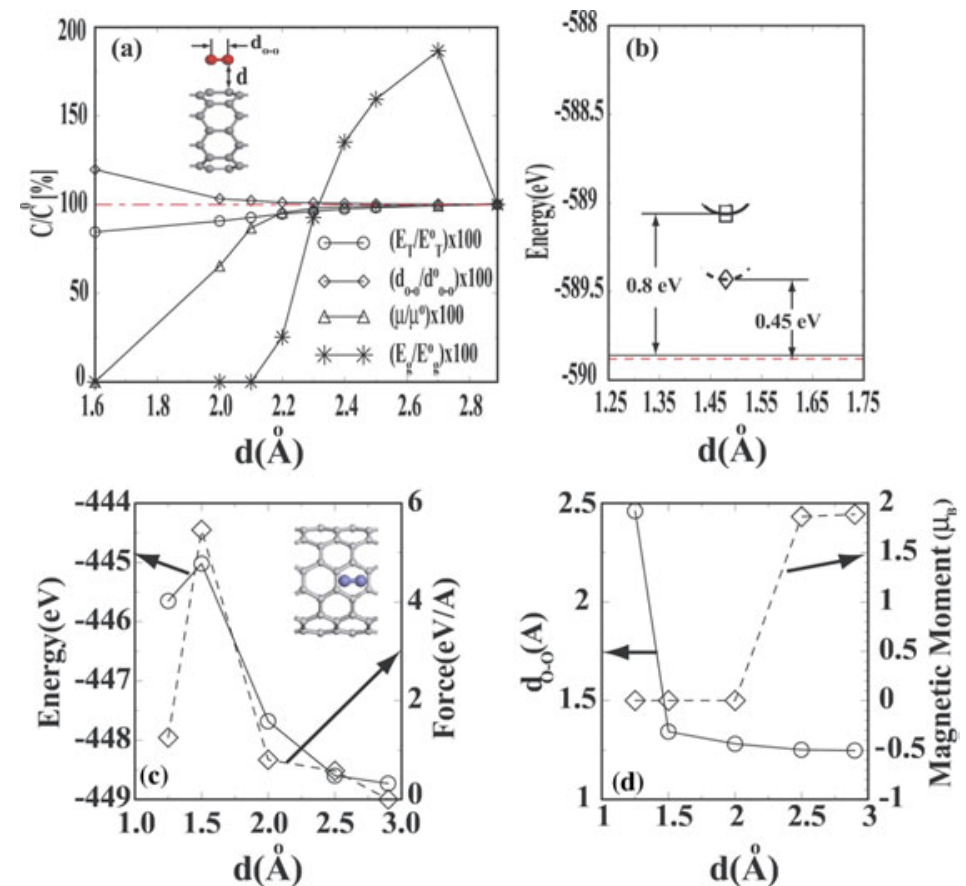

Figure 10. (a)Variation of the percentage values of $\mathbf{C} / \mathbf{C}^{0}$ (i.e. $E_{\mathrm{T}} / E_{\mathrm{T}}^{0}, d_{\mathrm{O}-\mathrm{O}} / d_{\mathrm{O}-\mathrm{O}}^{0}, \mu / \mu^{0}$ and $E_{\mathrm{g}} / E_{\mathrm{g}}^{0}$ ) with the $\mathrm{O}_{2}-\mathrm{SWNT}$ separation $d . E_{\mathrm{T}}^{0}, d_{\mathrm{O}-\mathrm{O}}^{0}, \mu^{0}$ and $E_{\mathrm{g}}^{0}$ correspond to the stable physisorption state with $d^{0}=2.89 \AA$ at the A site. (b) The total energies of the singlet bound states found at small $d$ at the $\mathrm{Z}$ site (square) and at the $\mathrm{A}$ site (diamond). The total energies of the triplet ground state corresponding to the physisorption state for $\mathrm{Z}$ and $\mathrm{A}$ sites at $d \sim 2.9 \AA$ are shown by continuous and broken lines. (c) Variation of the total energy, $E_{\mathrm{T}}$, and the force acting on the $\mathrm{O}_{2}$ molecule, $F_{\perp}$; (d) variation of the bond distance of $\mathrm{O}_{2}, d_{\mathrm{O}-\mathrm{O}}$, and the magnetic moment, $\mu$, with the $\mathrm{O}_{2}-(6,6)$ SWNT distance $d$ for $\mathrm{O}_{2}$ adsorbed on the $\mathrm{H}$ site of the $(6,6)$ SWNT. (Reproduced from [77].)

is energetically most favourable with $E_{\mathrm{s}}=5.07 \mathrm{eV}$. The binding energies as large as $\sim 5 \mathrm{eV}$ suggest that atomic $\mathrm{O}$ is actually chemisorbed with a significant charge transfer from $\mathrm{C}$ to $\mathrm{O}$. Moreover, the energy gained from the chemisorption of two atomic oxygens is more than the bond energy of $\mathrm{O}_{2}$ in either magnetic or nonmagnetic states. This implies that the dissociation of $\mathrm{O}_{2}$ followed by the chemisorption of individual $\mathrm{O}$ atoms is an exothermic process similar to other oxidation processes. As a manifestation of the curvature effect, the strained $\mathrm{C}-\mathrm{C}$ bond at the $\mathbf{z}$ site is broken, but the $\mathrm{C}-\mathrm{O}$ bond is strengthened. Experimentally, it was shown that oxygen exposure first oxidizes and eventually etches away the nanotubes with smaller radius [87].

The electronic state of an oxygen atom chemisorbed on a SWNT depends on the coverage and pattern of adsorption. For example, since a single oxygen (per unit cell) chemisorbed at an a site gives rise to flat bands below $E_{\mathrm{V}}$, the band gap of the $(8,0)$ SWNT remains unaffected. However, as a well known and usual effect of oxygen, the band gap of the $(8,0)$ tube opens up to $3.64 \mathrm{eV}$ when all a sites are filled by chemisorbed oxygen atoms [75].

\section{Adsorption of individual atoms on SWNTs}

This sections deals with the adsorption of individual atoms on SWNTs and the resulting properties. Because of miniaturization aiming at higher and higher device densities, the 
$\mathbf{a}$

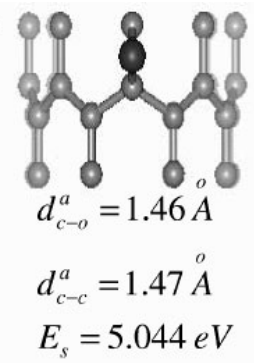

$\mathbf{Z}$

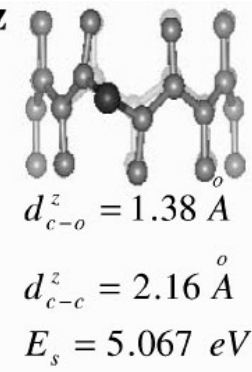

c

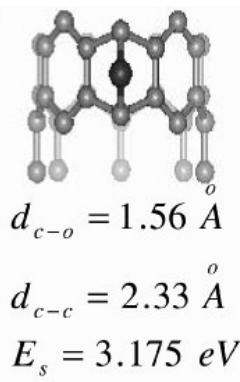

aa

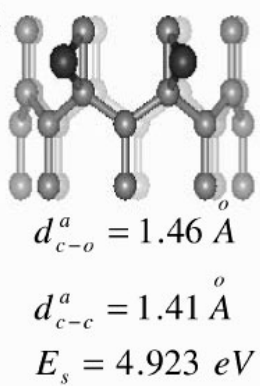

$\mathbf{Z Z}$

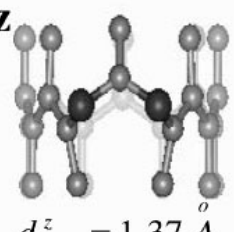

$d_{c-o}^{z}=1.37 \mathrm{~A}$

$d_{c-c}^{z}=2.24 \stackrel{\circ}{A}$

$E_{s}=5.284 \mathrm{eV}$ az1

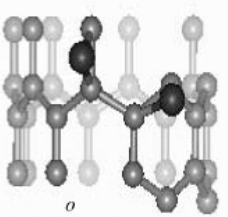

$d_{c-o}^{a}=1.45 \stackrel{\circ}{A}, d_{c-o}^{z}=1.44 \AA^{\circ}$

$d_{c-c}^{a}=1.46 \stackrel{\circ}{A}, d_{c-c}^{z}=1.53 \stackrel{\circ}{A}$

$E_{s}=4.939 \mathrm{eV}$

\section{az2}

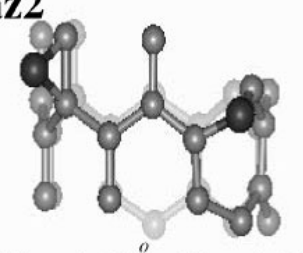

aa1

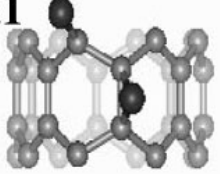

$d_{c-o}^{a}=1.45 \stackrel{\circ}{\AA}, d_{c-o}^{z}=1.38 \AA$

$d_{c-o}^{z}=1.45 \stackrel{o}{A}$

$d_{c-c}^{a}=1.47 \stackrel{\circ}{A}, d_{c-c}^{z}=2.15 \stackrel{\circ}{A}$

$d_{c-c}^{z}=1.46 \stackrel{o}{A}$

$E_{s}=5.256 \mathrm{eV}$

azz

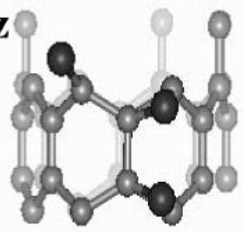

$d_{c-o}^{a}=1.45 \stackrel{\circ}{A}, d_{c-o}^{z}=1.40 \stackrel{\circ}{A}$

$d_{c-c}^{a}=1.46 \stackrel{o}{A}, d_{c-c}^{z}=1.51 \stackrel{o}{A}$

$E_{\mathrm{s}}=4.635 \mathrm{eV}$

Figure 11. A schematic illustration of the various adsorption sites of atomic $\mathrm{O}$ on the $(8,0) \mathrm{SWNT}$. Some relevant geometrical data and GGA chemical bond (chemisorption) energies corresponding to these sites are also given. $E_{\mathrm{s}}$ : chemisorption energy; $d_{\mathrm{C}-\mathrm{O}}$ : length of the $\mathrm{C}-\mathrm{O}$ bond; $d_{\mathrm{C}-\mathrm{C}}$ : length of the $\mathrm{C}-\mathrm{C}$ bond under an adsorbed $\mathrm{O}$ atom. $E_{\mathrm{s}}$ is obtained from the spin unpolarized calculations of the total energies in equation (3). (Reproduced from [75].)

realization of interconnects with high conductance and low energy dissipation appears to be a real technological challenge. Very thin metal wires and atomic chains were produced by retracting an STM tip from an indentation and then thinning the neck of the material that wets the tip [88-90]. While the nanowires produced so far have played a crucial role in understanding the quantum effects in electronic and thermal conductance [14,91-94], they were not reproducible enough to offer any relevant technological application. Nowadays, the most practical and realizable method for fabricating nanowires relies on carbon nanotubes. Moreover, electron transport measurements showed that SWNTs can resist high Joule heating [95].

Earlier experimental studies have indicated that SWNTs can serve as templates for producing reproducible, very thin metallic wires with controllable sizes [96]. These metallic nanowires can be used as conducting connects and hence are important in nanodevices based on molecular electronics. Recently, Zhang et al [97] have shown that continuous Ti coating of 


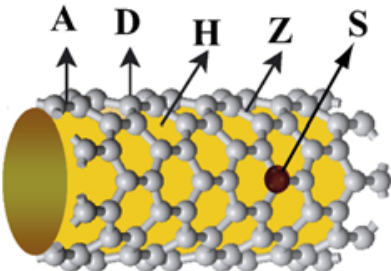

$(8,0)$ Zigzag SWNT

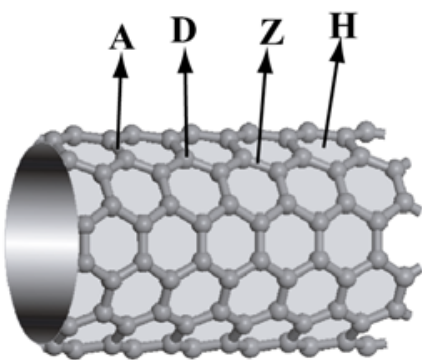

$(6,6)$ Armchair SWNT

Figure 12. A schematic illustration of different binding sites of individual atoms adsorbed on a zigzag $(8,0)$ tube. H: hollow; A: axial; Z: zigzag; D: top; S: substitution sites. (Reproduced from [103].)

varying thickness and quasi-continuous coating of $\mathrm{Ni}$ and $\mathrm{Pd}$ can be obtained by using electron beam evaporation techniques. However, metal atoms such as $\mathrm{Au}, \mathrm{Al}, \mathrm{Fe}, \mathrm{Pb}$ were able to form only isolated discrete patterns or clusters rather than a continuous coating of the SWNT.

Not only metallic connects, but also the contacts of metal electrodes themselves are crucial for the operation of devices based on nanotubes. Low resistance ohmic contacts to metallic and semiconducting SWNTs were achieved by Ti and Ni [98]. The formation of Schottky barrier at the contact has been found to be responsible for the operation of field emission transistors made from SWNTs [99-101].

\subsection{Binding geometry and binding energies}

Recently, an extensive study on the binding geometry, binding energy and resulting electronic structure of various atoms (ranging from alkali and simple metals to group IV elements, and including most of the transition metal atoms) adsorbed on SWNTs has been carried out by Durgun et al $[102,103]$. They considered four possible sites shown in figure 12, and determined the binding geometry and binding energies by optimizing all atomic positions.

The binding energy is calculated from the short range (chemical) interaction using the expression $E_{\mathrm{b}}^{\mathrm{u}(\mathrm{p})} \simeq E_{\mathrm{s}}^{\mathrm{u}(\mathrm{p})}=E_{\mathrm{T}}^{\mathrm{u}(\mathrm{p})}[\mathrm{SWNT}]+E_{\mathrm{T}}^{\mathrm{u}(\mathrm{p})}[\mathrm{A}]-E_{\mathrm{T}}^{\mathrm{u}(\mathrm{p})}[\mathrm{A}+\mathrm{SWNT}]$, similar to that given in equation (3). Here the total energy of the adsorbed atom is $E_{\mathrm{T}}^{\mathrm{u}(\mathrm{p})}$ [A]. The superscript $\mathrm{u}(\mathrm{p})$ indicates spin unpolarized (spin polarized) energies. The binding energies $E_{\mathrm{s}}^{\mathrm{u}(\mathrm{p})}$ are obtained from the total energies corresponding to either a nonmagnetic (spin unpolarized) state with zero net spin or a spin relaxed state. The long range interaction, $E_{\mathrm{vdW}}$, is expected to be much smaller than the chemisorption binding energy and is omitted. However, for specific elements, such as $\mathrm{Mg}$ and $\mathrm{Zn}$, the binding energy is small and the character of the bond is between chemisorption and physisorption. Tables 2 and 3 summarize the results relating to the adsorption of individual atoms.

The interaction between the SWNT and most of the adatoms in tables 2 and 3 is significant and results in a chemisorption bond. Thus, the binding energies corresponding to a nonmagnetic state range from $\sim 1$ to $\sim 4.5 \mathrm{eV}$. While alkali and simple metals have binding energy in the range of $1.5 \mathrm{eV}$, the chemisorption energy of transition metals is relatively high. On the other hand, metals such as $\mathrm{Cu}, \mathrm{Au}, \mathrm{Ag}$ and $\mathrm{Zn}$ have relatively weak binding. The centre of the hexagons ( $\mathrm{H}$ site) appears to be favoured by most of the adatoms. The average $\mathrm{C}$-adatom bond distance occurs in the range of 2.0-2.3 $\AA$. However, $\bar{d}_{\mathrm{C}-\mathrm{A}}$ is relatively small for $\mathrm{H}, \mathrm{C}, \mathrm{O}$ atoms having small atomic radii. It is well known that the 
Table 2. The strongest binding site (shown in figure 12); the adsorbate-carbon distance $\bar{d}_{\mathrm{C}-\mathrm{A}}$; the difference between spin unpolarized and spin polarized total energies $\Delta E_{\mathrm{T}}$; the binding energy $E_{\mathrm{b}}^{\mathrm{u}}$ obtained from spin unpolarized calculations; the binding energy $E_{\mathrm{b}}^{\mathrm{p}}$ obtained from spin polarized calculations; the magnetic moment $\left(\mu_{\mathrm{B}}\right.$ /supercell) of the magnetic ground state corresponding to the adsorption of various individual atoms on the $(8,0)$ SWNT. (Reproduced from [103].)

\begin{tabular}{lllllll}
\hline Atom & Site & $\bar{d}_{\mathrm{C}-\mathrm{A}}(\AA)$ & $\Delta E_{\mathrm{T}}(\mathrm{eV})$ & $E_{\mathrm{b}}^{\mathrm{u}}(\mathrm{eV})$ & $E_{\mathrm{b}}^{\mathrm{p}}(\mathrm{eV})$ & $\mu\left(\mu_{\mathrm{B}}\right)$ \\
\hline $\mathrm{Na}$ & $\mathrm{H}$ & 2.3 & - & - & 1.3 & - \\
$\mathrm{Sc}$ & $\mathrm{H}$ & 2.2 & 0.15 & 2.1 & 1.9 & 0.64 \\
$\mathrm{Ti}$ & $\mathrm{H}$ & 2.2 & 0.58 & 2.9 & 2.2 & 2.21 \\
$\mathrm{~V}$ & $\mathrm{H}$ & 2.2 & 1.20 & 3.2 & 1.4 & 3.67 \\
$\mathrm{Cr}$ & $\mathrm{H}$ & 2.3 & 2.25 & 3.7 & 0.4 & 5.17 \\
$\mathrm{Mn}$ & $\mathrm{H}$ & 2.4 & 2.42 & 3.4 & 0.4 & 5.49 \\
$\mathrm{Fe}$ & $\mathrm{H}$ & 2.3 & 1.14 & 3.1 & 0.8 & 2.27 \\
$\mathrm{Co}$ & $\mathrm{H}$ & 2.0 & 0.41 & 2.8 & 1.7 & 1.05 \\
$\mathrm{Ni}$ & $\mathrm{A}$ & 1.9 & 0.02 & 2.4 & 1.7 & 0.04 \\
$\mathrm{Cu}$ & $\mathrm{A}$ & 2.1 & 0.03 & 0.8 & 0.7 & 0.53 \\
$\mathrm{Zn}$ & $\mathrm{H}$ & 3.7 & 0 & 0.05 & 0.04 & 0 \\
$\mathrm{Nb}$ & $\mathrm{H}$ & 2.2 & 0.40 & 3.9 & 1.8 & 2.86 \\
$\mathrm{Mo}$ & $\mathrm{H}$ & 2.2 & 0.32 & 4.6 & 0.4 & 4 \\
$\mathrm{Pd}$ & $\mathrm{A}$ & 2.1 & 0 & 1.7 & 1.7 & 0 \\
$\mathrm{Ag}$ & $\mathrm{A}$ & 2.5 & 0.03 & 0.3 & 0.2 & 0.6 \\
$\mathrm{Ta}$ & $\mathrm{H}$ & 2.2 & 0.73 & 2.9 & 2.4 & 3.01 \\
$\mathrm{~W}$ & $\mathrm{H}-\mathrm{A}$ & 2.1 & 0.59 & 3.4 & 0.9 & 2.01 \\
$\mathrm{Pt}$ & $\mathrm{A}$ & 2.1 & 0 & 2.7 & 2.4 & 0 \\
$\mathrm{Au}$ & $\mathrm{A}-\mathrm{T}$ & 2.2 & 0.04 & 0.6 & 0.5 & 1.02 \\
$\mathrm{Al}$ & $\mathrm{H}$ & 2.3 & - & 1.6 & - & - \\
$\mathrm{C}$ & $\mathrm{Z}$ & 1.5 & - & 4.2 & - & - \\
$\mathrm{Si}$ & $\mathrm{H}$ & 2.1 & - & 2.5 & - & - \\
$\mathrm{Pb}$ & $\mathrm{H}$ & 2.6 & 0.01 & 1.3 & 0.8 & 0 \\
$\mathrm{H}$ & $\mathrm{T}$ & 1.1 & - & 2.5 & - & - \\
$\mathrm{O}$ & $\mathrm{Z}$ & 1.5 & - & 5.1 & - & - \\
$\mathrm{S}$ & $\mathrm{A}$ & 1.9 & - & 2.8 & - & - \\
\hline & & & - & & & \\
\hline
\end{tabular}

Table 3. The strongest binding site (as shown in figure 12); the adsorbate-carbon distance $\bar{d}_{\mathrm{C}-\mathrm{A}}$; the difference between spin unpolarized and spin polarized total energies $\Delta E_{\mathrm{T}}$; the binding energy $E_{\mathrm{b}}^{\mathrm{u}}$ obtained from spin unpolarized calculations; the binding energy $E_{\mathrm{b}}^{\mathrm{p}}$ obtained from spin polarized calculations; the magnetic moment $\mu$ per supercell corresponding to the magnetic ground state corresponding to the adsorption of individual Ti, Mn, Mo, Au atoms on a (6, 6) SWNT. (Reproduced from [103].)

\begin{tabular}{lllllll}
\hline Atom & Site & $\bar{d}_{\mathrm{C}-\mathrm{A}}(\AA)$ & $\Delta E_{\mathrm{T}}(\mathrm{eV})$ & $E_{\mathrm{b}}^{\mathrm{u}}(\mathrm{eV})$ & $E_{\mathrm{b}}^{\mathrm{p}}(\mathrm{eV})$ & $\mu\left(\mu_{\mathrm{B}}\right)$ \\
\hline $\mathrm{Ti}$ & $\mathrm{H}$ & 2.2 & 0.48 & 2.62 & 1.81 & 1.68 \\
$\mathrm{Mn}$ & $\mathrm{H}$ & 2.5 & 2.23 & 3.25 & 0.1 & 5.60 \\
$\mathrm{Mo}$ & $\mathrm{H}$ & 2.3 & 0.2 & 4.34 & 0.1 & 3.61 \\
$\mathrm{Au}$ & $\mathrm{T}$ & 2.3 & 0.02 & 0.41 & 0.28 & 0.79 \\
\hline
\end{tabular}

interaction between the graphite surface and most of the atoms included is actually weak. The curvature effect is the primary factor that strengthens the binding [18, 26, 43]. Specific adsorbate-SWNT $(\mathrm{A}+\mathrm{SWNT})$ systems are found to be in a magnetic ground state, since $\Delta E_{\mathrm{T}}=E_{\mathrm{T}}^{\mathrm{u}}[\mathrm{A}+\mathrm{SWNT}]-E_{\mathrm{T}}^{\mathrm{p}}[\mathrm{A}+\mathrm{SWNT}]>0$. Most of the transition metal atoms adsorbed on the $(8,0)$ and $(6,6)$ tubes are in the magnetic ground state with $\Delta E_{\mathrm{T}}>0$, and hence they have net magnetic moments ranging from $5.49 \mu_{\mathrm{B}}$ (for $\mathrm{Mn}$ ) to zero magnetic moment (for $\mathrm{Pd}$ 
and $\mathrm{Pt})$. While Ni adsorbed SWNT has very low magnetic moment $\left(0.04 \mu_{\mathrm{B}}\right)$, the adsorbates such as $\mathrm{Au}, \mathrm{Ag}$ and $\mathrm{Cu}$ have magnetic moments in the range of 0.4-0.6 $\mu_{\mathrm{B}}$. Atoms, such as $\mathrm{Na}, \mathrm{Al}, \mathrm{C}, \mathrm{Si}, \mathrm{Pb}, \mathrm{O}, \mathrm{S}, \mathrm{H}$ favour a nonmagnetic ground state when they are adsorbed on the $(8,0)$ SWNT. The magnetic moment generated upon the adsorption of individual transition atoms has important implications, and points to an issue: whether molecular magnets (or nanomagnets) can be produced from carbon nanotubes. Addressing this issue may open an active field of study on SWNTs, which are covered or substitutionally doped by transition metal atoms according to a well defined pattern. Inclusion of transition metal elements inside the tube is another way to obtain nanomagnetic structures. In this way, these atoms are prevented from oxidizing. Whether a permanent magnetic moment can be generated by exchange interaction on these transition metal coated SWNTs would be an interesting question to answer. Recently, the magnetization and hysteresis loops of iron nanoparticles partially encapsulated at the tips and inside of aligned carbon nanotubes have been demonstrated by experimental works [104].

Binding energies listed in table 2 are of particular interest for coating of SWNTs by metal atoms, and hence for the fabrication of nanowires. The atoms which were observed to form continuous and quasi-continuous coating on the SWNT (Ti, Ni and Pd) have relatively high binding energies as compared to those atoms $(\mathrm{Au}, \mathrm{Fe}, \mathrm{Pb})$ which remain as discrete particles on the surface of the tube [96]. We also note that in forming a good coverage, not only adatom-SWNT interaction, but also other factors, possibly adatom-adatom interaction, play a crucial role. Good conductors such as $\mathrm{Au}, \mathrm{Ag}$, and $\mathrm{Cu}$ have very weak binding. On the other hand, $\mathrm{Na}$ with one $3 \mathrm{~s}$ electron on the outer shell is bound with a significant binding energy $\left(E_{\mathrm{b}}=1.3 \mathrm{eV}\right)$. The binding energy of $\mathrm{Mg}$ is very weak and is only $0.03 \mathrm{eV}$ at the $\mathrm{H}$ site due to its outer shell $(3 \mathrm{~s})^{2}$. For the same reason $\mathrm{Zn}$ by itself exhibits a similar trend $\left(E_{\mathrm{b}}^{\mathrm{u}}=0.05 \mathrm{eV}\right)$ with its $(4 \mathrm{~s})^{2}$ valence structure. Owing to the weak binding the type of the bond between $\mathrm{Mg}(\mathrm{Zn})$ and the SWNT is between chemisorption and physisorption with $d_{\mathrm{C}-\mathrm{A}}=3.8 \AA\left(d_{\mathrm{C}-\mathrm{A}}=3.7 \AA\right)$. While an individual $\mathrm{Al}$ atom (with $(3 \mathrm{~s})^{2} 3 \mathrm{p}$ valence structure) is not bound to the graphite surface, its binding on the $(8,0)$ SWNT is relatively strong. This can be explained by the curvature effect, since the binding was found to be even stronger at the high curvature site of the SWNT under uniaxial radial deformation $[26,43]$. Note also that the binding energies $E_{\mathrm{b}}^{\mathrm{u}}$, as well as $E_{\mathrm{b}}^{\mathrm{p}}$ and the magnetic moments of the adatom adsorbed on the $(8,0)$ SWNT, came out to be consistently lower for the adatom adsorbed on the $(6,6)$ tube. Perhaps this trend can also be explained by the curvature effect, since the radius of $(8,0)$ is smaller than that of $(6,6)[18,31]$.

The transition metal atoms with a few d electrons, such as $\mathrm{Sc}, \mathrm{Co}, \mathrm{Ti}, \mathrm{Nb}, \mathrm{Ta}$, form strong bonds with a binding energy ranging from 2.4 to $1.8 \mathrm{eV}$, and hence can be suitable for metal coating of a SWNT. These metals can also be used as a buffer layer to form uniform coating of good conductors such as $\mathrm{Au}, \mathrm{Ag}, \mathrm{Cu}$. Most of the adatoms yield the strongest binding at the $\mathrm{H}$ site. Ni, Pd, Pt (column VIII elements) and $\mathrm{Cu}, \mathrm{Ag}, \mathrm{Au}$ (column I-B elements) seem to prefer the A site. The average adsorbate- $\mathrm{C}$ distance, $\bar{d}_{\mathrm{C}-\mathrm{A}}$, ranges between $1.9 \AA$ (minimum) and $3.7 \AA$ (maximum); most of them occur at $\sim 2.1 \AA$. The interaction of group IV elements with the SWNT is also crucial, since they have the same valence configuration. C and Si adatoms form rather strong bonds with the SWNT. The calculated binding energies are rather high, i.e. $E_{\mathrm{b}}=4.2$ and $2.5 \mathrm{eV}$, respectively. The $\mathrm{Z}$ site is energetically favourable for both $\mathrm{C}$ and $\mathrm{Si}$ adatoms.

The character of the bond between the adatom and SWNT can be examined by charge density analysis. For the fully relaxed, minimum total energy configuration, the SCF total charge density $\rho(\mathbf{r})=\sum_{n, k}^{\text {occup }} \Psi_{n, k}^{*}(\mathbf{r}) \Psi_{n, k}(\mathbf{r})$ and the difference charge density $\Delta \rho(\mathbf{r})=$ $\rho[\mathrm{A}+\mathrm{SWNT}]-\rho[\mathrm{SWNT}]-\rho[\mathrm{A}]$ can be calculated. In $\Delta \rho(\mathbf{r})$, the total charge densities of adatom adsorbed SWNT $\rho$ [A + SWNT], of bare SWNT $\rho$ [SWNT] and of free atom $\rho[\mathrm{A}]$ 

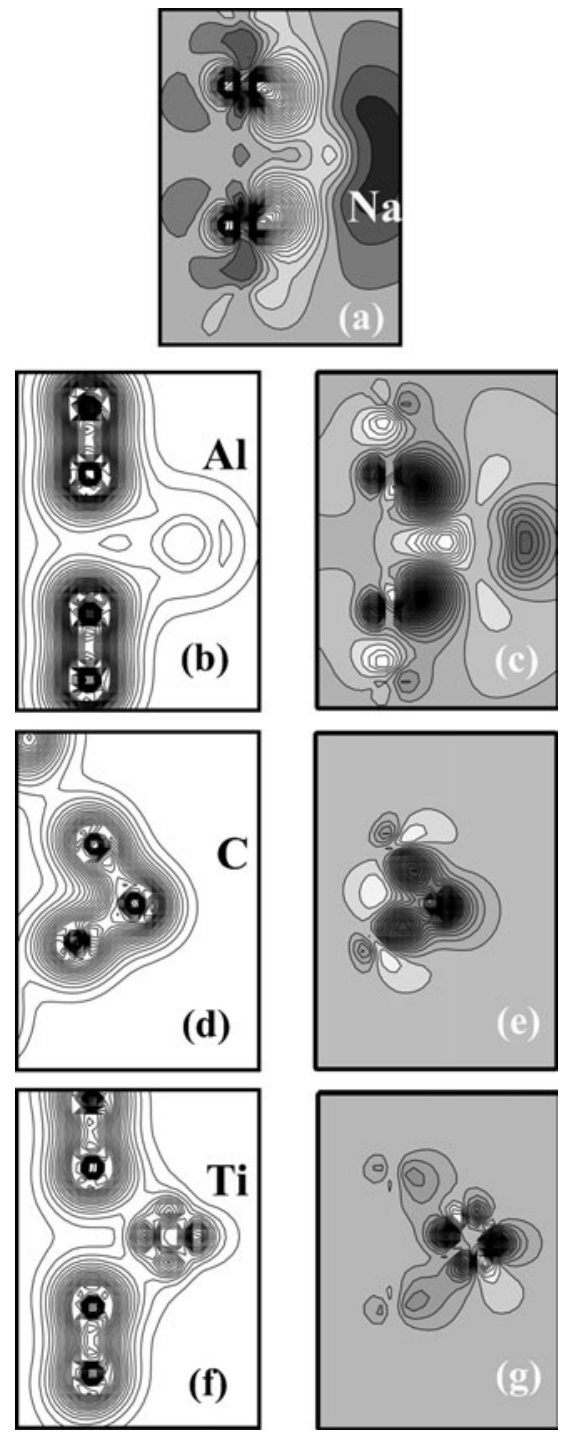

Figure 13. Contour plots of total $\rho(\mathbf{r})$ and difference $\Delta \rho(\mathbf{r})$ charge densities. For $\mathrm{C}$ the charge densities are calculated on a plane passing through the adatom and zigzag $\mathrm{C}-\mathrm{C}$ bond. For others ( $\mathrm{Na}, \mathrm{Al}, \mathrm{Ti})$ the charge density plane passes through the centre of the hexagon and adatom. In contour plots of $\Delta \rho(\mathbf{r})$, charge is depleted from black regions and is accumulated at white regions. The counter-plots of $\rho(\mathbf{r})$ for $[\mathrm{Na}+\mathrm{SWNT}]$ are not presented, since they do not convey relevant information owing to the low charge density around adsorbed Na atoms. (Reproduced from [103].)

are all calculated in the same supercell with the same atomic positions. The difference charge density conveys information about the charge rearrangements upon adsorption. Figure 13 shows $\rho(\mathbf{r})$ and $\Delta \rho(\mathbf{r})$ which were calculated for four adatoms, namely $\mathrm{Na}, \mathrm{Al}, \mathrm{C}, \mathrm{Ti}$ [103]. Dramatic differences in the valence electron configurations of these adatoms are reflected in the characters of the bonds they form with the SWNT.

According to the above analysis the charge is generally transferred from the adatom to the $\mathrm{C}-\mathrm{C}$ bond of SWNT. The $3 \mathrm{~s}$ valence electron of $\mathrm{Na}$ is weakly bound and is donated to the 
conduction band of the SWNT, which is derived from the modified conduction band of the bare SWNT. In the case of $\mathrm{Al}$, which is adsorbed at the $\mathrm{H}$ site, electrons are transferred from $\mathrm{Al}$ and nearest $\mathrm{C}$ atoms to the region between the $\mathrm{Al}$ atom and the centre of the hexagon on the surface of the SWNT and to the $\mathrm{C}-\mathrm{C}$ bond. The character of the bond formed between the $\mathrm{C}$ adatom and $\mathrm{C}$ atoms of the SWNT is reminiscent of the $\mathrm{sp}^{3}$ covalent bonds in diamond crystals. $\rho(\mathbf{r})$ has a maximum value at the centre of the $\mathrm{C}-\mathrm{C}$ bonds. Because of new bond formation, charge is transferred to the back-bonds. The charge density of adsorbed Si exhibits a character similar to that of adsorbed C. Directional bonds form between Si and C atoms of the SWNT. The Ti atom adsorbed at the $\mathrm{H}$ site forms a bond. The $\mathrm{d}$ orbitals are responsible for this bond. According to the Mulliken analysis the charge transferred from a Ti atom is 1.45 electrons for the spin unpolarized case. The interaction between the Au atom and SWNT is weak, which results in a small binding energy. Accordingly, the charge rearrangement due to Au adsorption is minute.

\subsection{Electronic structure}

An individual atom adsorbed on an SWNT may give rise to resonance states in the valence and conduction bands, and also localized states in the band gaps. The energy states associated with a single adsorbate form energy bands if the electronic structure is calculated within the supercell method. Actually these bands correspond to a linear chain of adsorbates with a 1D lattice constant of the supercell, $c_{\mathrm{s}}$. The localized states are relevant for the doping of a s-SWNT. Depending on their position relative to the band edges, they are specified as donor states (if they are close to the edge of the conduction band, $E_{\mathrm{C}}$ ) or as acceptor states (if they can occur close to the edge of the valence band, $E_{\mathrm{V}}$ ). The latter case is also known as hole doping.

$\mathrm{Na}, \mathrm{Al}, \mathrm{C}$ and $\mathrm{Si}$, which are adsorbed individually on the $(8,0) \mathrm{SWNT}$ with a repeat period of $2 c$, give rise to a spin paired, nonmagnetic ground state [103]. Figure 14 presents their calculated band structures and the LDOS at the adatoms. The calculated band structure of the SWNT with $\mathrm{Na}$ adsorbed at the $\mathrm{H}$ site forming a regular chain with a lattice parameter (or interatomic distance) $a=c_{\mathrm{s}} \sim 2 c$ yields a half-filled band. This band is normally the first empty conduction band of the tube. Since the $3 \mathrm{~s}$ valence electron of $\mathrm{Na}$ has low ionization energy, it is donated to the SWNT to occupy the empty conduction band. As a result, this empty band is gradually populated and also modified upon adsorption of $\mathrm{Na}$; hence the SWNT becomes metallic. Another alkali metal, Li, has been the focus of attention because of SWNT ropes which were expected to be a candidate for anode material use in Li battery applications [105]. In fact, recent experiments showed higher Li capacity in SWNTs than that of graphite [106]. Zhao et al [107] investigated Li intercalation in $(10,0),(12,0),(8,0)$ and $(10,10)$ SWNT ropes modelled by a uniform, 2D hexagonal lattice. They found that charge is transferred from adsorbed Li to the SWNT. Furthermore, the SWNT is slightly deformed upon $\mathrm{Li}$ adsorption. The band structure of the $\mathrm{Li}$ intercalated $(8,0)$ rope has indicated that the bands of the bare SWNTs are not affected in any essential manner; but their conduction bands get occupied above $E_{\mathrm{g}}$ and the system becomes metallic. Their findings are consistent with the adsorption of $\mathrm{Na}$ discussed above.

Similarly, the calculated band structure of the SWNT with Al adsorbed at the $\mathrm{H}$ site forming a regular chain structure gives rise to a half-filled band derived from the empty conduction band of the SWNT. Therefore, the localized state due to an adsorbed individual Al atom is a donor state. In the case of $\mathrm{C}$, a small gap occurs between the bands derived from adsorbate states. The empty and filled bands just above and below $E_{\mathrm{F}}$ are derived from $\mathrm{C}$ adatoms with a significant contribution from the SWNT. Si yields an almost fully occupied band in the band 

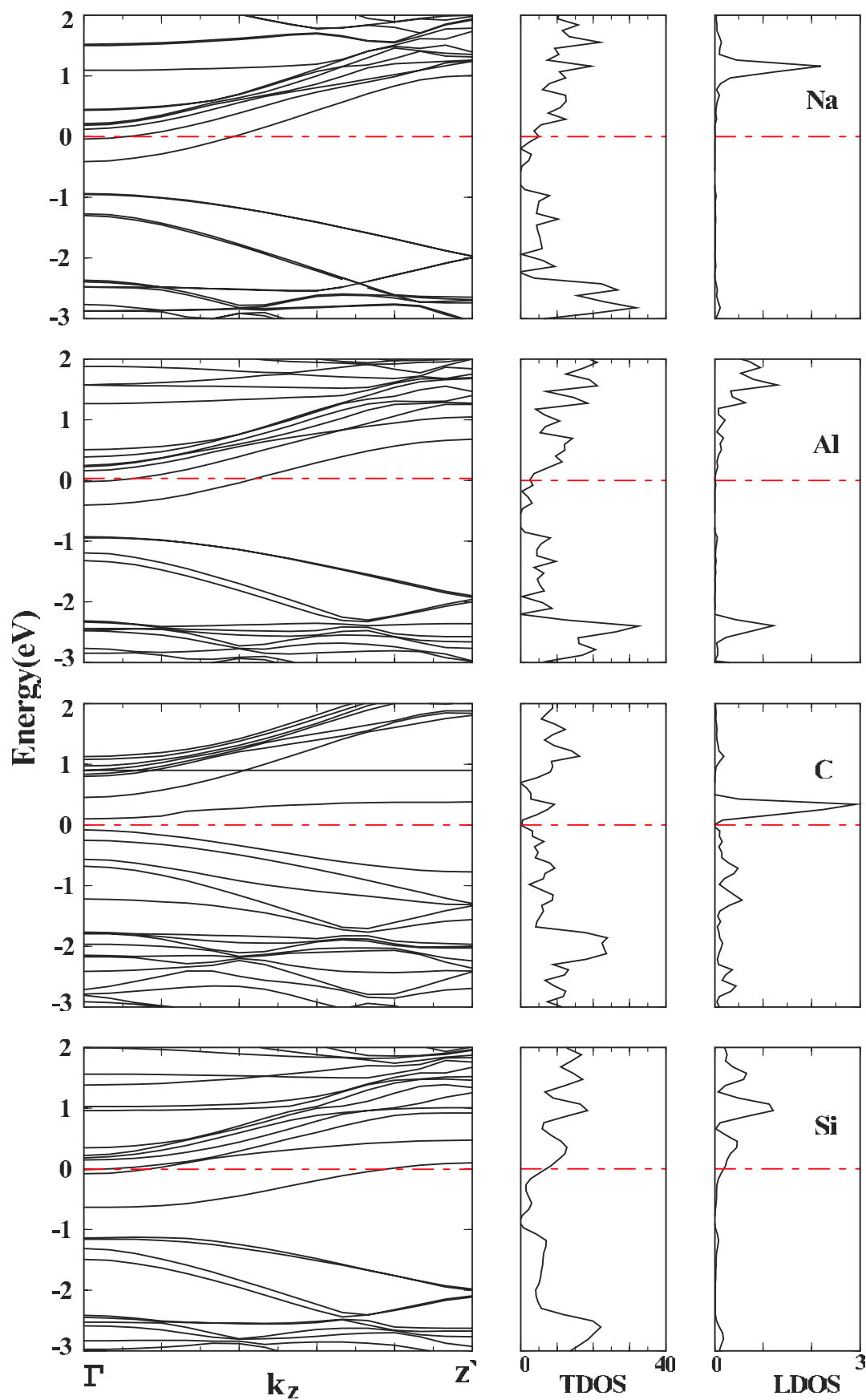

Figure 14. Energy band structures, TDOSs and LDOSs of single $\mathrm{Na}, \mathrm{Al}, \mathrm{C}, \mathrm{Si}$ adsorbed on a zigzag $(8,0)$ tube. LDOSs calculated at the adsorbate. The zero of energy is set at the Fermi level. $\mathrm{Na}, \mathrm{Al}$ and $\mathrm{Si}$ are adsorbed at the $\mathrm{H}$ site; $\mathrm{C}$ is adsorbed at the $\mathrm{Z}$ site. (Reproduced from [103].)

gap and an almost empty band at the bottom of the conduction band. The dispersion of bands for $\mathrm{Si}$ adsorbed on the $\mathrm{H}$ site displays differences from that of the $\mathrm{C}$ adatom adsorbed at the $\mathrm{Z}$ site. However, the overall behaviours of state densities are similar. 

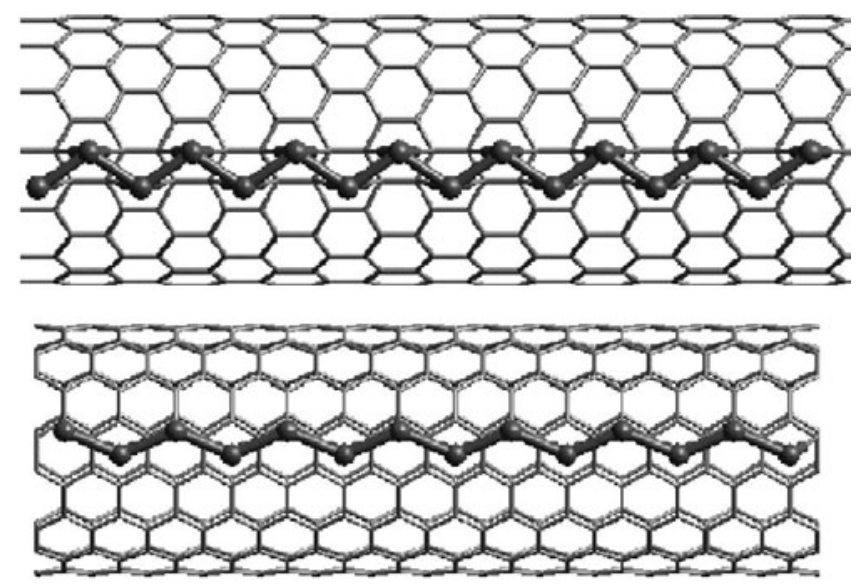

Figure 15. Geometric structures of adsorbed Ti chains on $(14,0)$ (top) and (8, 8) (bottom) SWNTs. Along the tube axis, each unit cell contains two Ti atoms for the $(14,0)$ tube and one Ti atom for the $(8,8)$ tube. Several unit cells are shown for the purpose of visualization. The distances between the two neighbouring Ti atoms in the chains are $2.6 \AA$ for $(14,0)$ and $2.7 \AA$ for $(8,8)$ tubes. (Reproduced from [108].)

The spin polarized band structure and TDOSs of adsorbed transition metal atoms (i.e. Au, Mn, Mo and Ti) display a different situation due to the magnetic ground state. Au yields two bands in the band gap, namely filled spin up and empty spin down bands. This is in compliance with the calculated magnetic moment of $\sim 1 \mu_{\mathrm{B}}$ per adsorbed Au atom. There is a small band gap of $\sim 0.2 \mathrm{eV}$ between these Au bands. It is seen that the adsorption of Au does not induce significant modification in the bands of $(8,0)$, except the Au $6 s(\uparrow)$ and Au $6 s(\downarrow)$ bands in the gap; it gives rise to two sharp peaks below and above $E_{\mathrm{F}}$ in the TDOS. That the contribution of the SWNT states to these peaks is minute and that the band gap between the conduction and valence bands of the SWNT is practically unchanged confirm the weak interaction between $\mathrm{Au}$ and the SWNT. Filled Au $5 \mathrm{~d}(\uparrow)$ and $5 \mathrm{~d}(\downarrow)$ bands occur in the valence band of the SWNT $2 \mathrm{eV}$ below $E_{\mathrm{F}}$. Three bands formed from Ti $3 \mathrm{~d}(\uparrow)$ are fully occupied and accommodate three electrons of adsorbed Ti atoms. Other Ti $3 \mathrm{~d}(\uparrow)$ bands occur above $E_{\mathrm{F}}$, but they overlap with the conduction band of the SWNT. The dispersive and almost fully occupied spin down band is formed from the states of carbon and hence derived from the conduction band of the bare SWNT. The SWNT is metallized upon Ti adsorption, since this band crosses the Fermi level and also overlaps with the other conduction bands. This situation is in accordance with the Mulliken analysis, which predicts the electrons to be transferred from Ti to the SWNT.

Yang et al [108] have studied the binding energies and electronic structures of $\mathrm{Ti}, \mathrm{Al}$ and Au chains adsorbed on $(10,0),(14,0),(6,6)$ and $(8,8)$ SWNTs using first-principles methods. The geometric configuration of the adsorbed metal chain is shown in figure 15. In spite of the fact that the calculated binding energies include significant coupling between adjacent metal atoms, they show the same trends as the binding energies given by Durgun et al [102, 103]. Ti is much more likely than $\mathrm{Al}$ and $\mathrm{Au}$ to form a continuous chain on the surface of SWNTs due to much higher binding energies. Al adsorbed SWNTs in turn have higher binding energies than the Au adsorbed ones. On the basis of the works by Bagci et al [109] and Prasen et al [110], we believe that the stability of the Al and Au chains originates from the stability of zigzag-free $\mathrm{Al}$ and $\mathrm{Au}$ chains. In calculating the binding energies the energy references are crucial. It is well known that adsorbed $\mathrm{Ti}$ and $\mathrm{Au}$ have magnetic ground states leading to a 


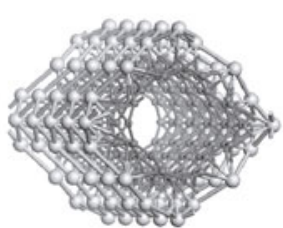

$(8,0)+\mathrm{Ti}$

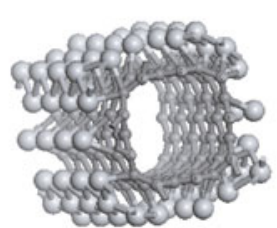

$(9,0)+\mathrm{Ti}$

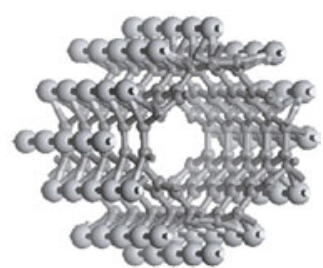

$(6,6)+\mathrm{Ti}$
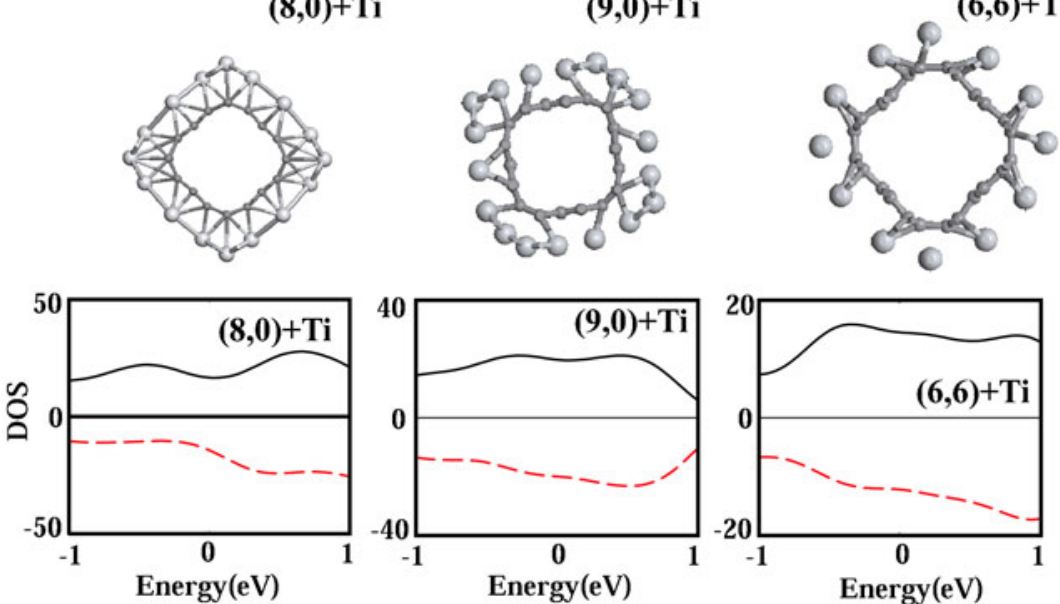

Figure 16. Optimized atomic structure of $(8,0),(9,0)$ and $(6,6)$ armchair SWNTs which are uniformly covered with Ti. Corresponding densities of states for spin up and spin down electrons are shown. (Reproduced from [7].)

net spin. Therefore, the binding energies reported by Yang et al [108] might change if one took the magnetic states into account. The important effect of the Ti chain is to metallize the zigzag SWNTs due to the delocalized d states crossing the Fermi level. However, the situation is different for the Ti chain adsorbed armchair tube, since a small gap is opened owing to the symmetry breaking.

\subsection{Transition element covered or filled SWNTs}

It is clear from the above discussion that individual atoms, such as $\mathrm{Ti}, \mathrm{Ni}, \mathrm{Si}$, can form rather strong bonds to the surface of the SWNT. Motivated by the experimental work of Zhang et al [97] showing the continuous coating of the SWNT by Ti, Dag et al [7] investigated the coverage of an $(8,0)$ tube by specific transition element atoms, such as $\mathrm{Ti}, \mathrm{Fe}, \mathrm{Co}, \mathrm{Cr}$, using a first-principles plane wave method. They found that only Ti can yield a continuous, regular coverage of the SWNT, as well as giving rise to a dramatic modification of the atomic structure. Upon optimization of the geometry the circular cross section of the SWNT has changed to a square. Even more interesting is that the s-SWNT has become a highly conducting metal due to several Ti bands crossing the Fermi level. The conductance of the Ti covered tube has been estimated to be $16 e^{2} / h$. Furthermore, they found that the Ti covered $(8,0),(9,0)$ and $(6,6)$ tubes have magnetic ground states with significant magnetic moments of $13.3,13.7$ and $9.5 \mu_{\mathrm{B}} /$ cell, respectively. Figure 16 shows the structure and density of states of Ti covered $(8,0),(9,0)$ and $(6,6)$ tubes.

Yang et al [111] have examined the magnetism of transition metal covered or filled $(9,0)$ SWNTs. They also found through ab initio calculations that these structures exhibit substantial 


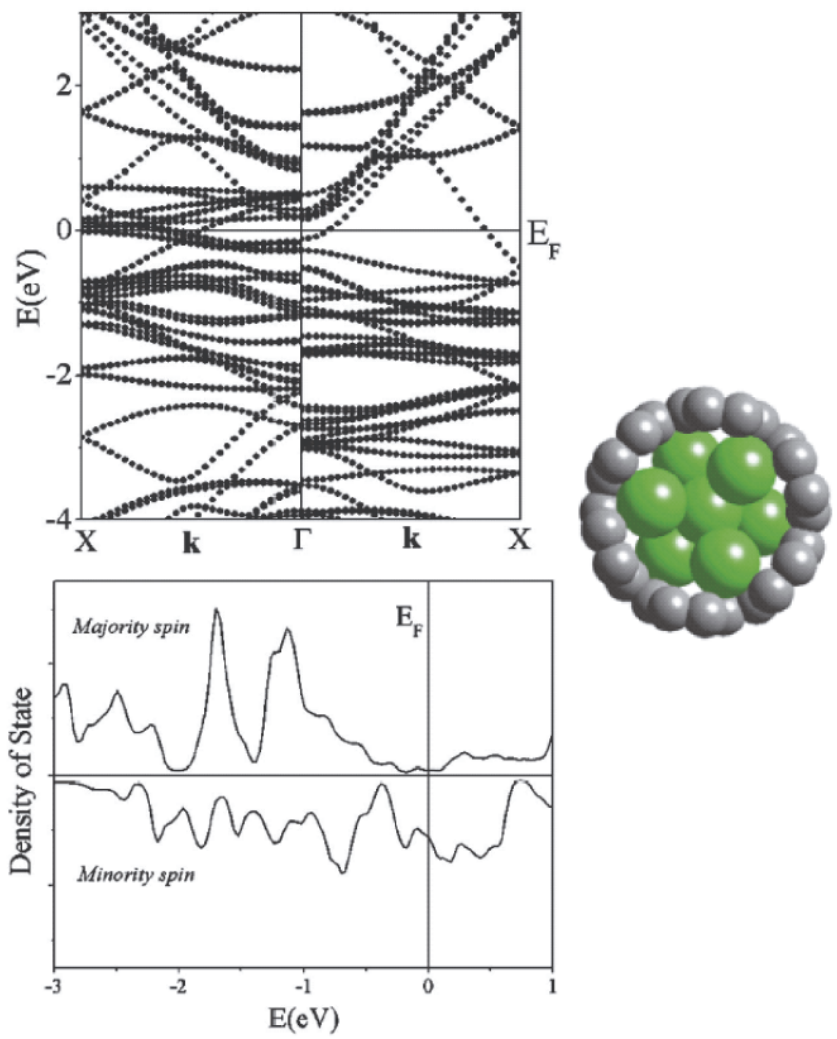

Figure 17. The spin polarized band structure (left: minority spin; right: majority spin) and DOS (bottom: minority spin; top: majority spin) for Co filled SWNT as shown in the right panel. Both plots suggest that the magnetic moment resides mostly in the narrow transition metal d band below the Fermi level. (Reproduced from [111].)

magnetism. In particular, Co atoms packed inside a variety of carbon nanotubes offer strong spin polarization at the Fermi level, as well as considerable magnetic moments. Figure 17 shows the spin polarized band structure and DOS for the Co filled SWNT.

\section{Radial deformation of carbon nanotubes}

Modification of electronic properties of condensed systems by an applied external pressure or strain in the elastic range has been a subject of active study. However, in most cases, the changes that one can induce by the elastic deformation are minute or even negligible due to the rigidity of the crystals. On the other hand, the situation is rather different for SWNTs owing to their tubular geometry [33]. Carbon nanotubes display very interesting mechanical properties. While the MWNTs show the greatest values for the Young's modulus, as high as 2 TPa, SWNTs appear to be very soft in the radial direction [112]. SWNTs are highly flexible: they can sustain elastic deformations remarkably. The softness of carbon nanotubes in the radial direction has been observed experimentally: the contact area is flattened when two nanotubes are brought close to each other [113]. If $R$ is large, a nanotube takes on a flattened structure [114]. It has been argued that SWNTs show linear elasticity under hydrostatic pressure up to $1.5 \mathrm{GPa}$ at room temperature. 
It has been shown that the structure and the electronic properties of SWNTs undergo dramatic changes as a result of these deformations [23, 43, 115-125]. Significant radial deformation of SWNTs can be realized in the elastic range, whereby the curvature is locally changed. In this way, zones with higher and lower curvatures relative to the undeformed SWNT can be attained for the same circumference. Hence, one expects radial deformation to induce important modifications in the electronic and conduction properties of nanotubes [24, 25, $126-$ 129]. Tight binding calculations have indicated that a SWNT may undergo an insulatormetal transition under a uniaxial or torsional strain [24, 124, 125]. Multiprobe transport experiments [23] on individual SWNTs showed that the electronic structure can be modified by bending the tube, or by applying a circumferential deformation. Empirical extended Hückel calculations [115] predicted that the conductance of an armchair SWNT can be affected by the circumferential deformations and a band gap can develop in a metallic armchair SWNT upon twisting.

A systematic analysis of the effect of the radial deformation on the electrical properties has been carried out by Gülseren et al [43] using first-principles total energy and electronic structure calculations. In their study the radial deformation is generated by applying uniaxial compressive stress $\sigma_{y y}$ to a narrow strip on the surface of a SWNT. In practice such a deformation can be realized by pressing the tube between two rigid and flat surfaces. As a result, the radius is squeezed in the $y$ direction while it is elongated along the $x$ direction and hence the circular cross section is distorted to an elliptical form with major and minor axes, $a$ and $b$, respectively. A natural variable for describing the radial deformation is the magnitude of the applied strain along the two axes,

$$
\epsilon_{y y}=\frac{R_{0}-b}{R_{0}}
$$

and

$$
\epsilon_{x x}=\frac{R_{0}-a}{R_{0}}
$$

where $R_{0}$ is the radius of the undeformed (zero-strain) nanotube. The point group of the undeformed nanotubes is $\mathrm{D}_{n \mathrm{~h}}$ or $\mathrm{D}_{n \mathrm{~d}}$ for $n$ even or odd, respectively. Under the radial deformation described above, the point group becomes $\mathrm{C}_{2 \mathrm{~h}}$ or $\mathrm{D}_{2 \mathrm{~h}}$. However, depending on the nanotube orientation around the tube axes, the in-plane mirror symmetry can be broken. For the $(6,6)$ tube, there are several different orientations to investigate when looking at the effect of mirror symmetry on the band crossing at the Fermi level. The first-principles calculations predict that elastic radial deformation does not have a noticeable effect on the first- and secondnearest neighbour $\mathrm{C}-\mathrm{C}$ distances but it induces significant changes in the bond angles [43]. This observation is therefore important and has to be taken into account in tight binding studies of SWNTs with radial deformation.

\subsection{Elasticity}

For the radial deformation, the relation between stress and strain is given by the generalized Hooke's law,

$$
\sigma_{x x}=0=C_{11} \epsilon_{x x}+C_{12} \epsilon_{y y}
$$

and

$$
\sigma_{y y}=\frac{F_{y}}{A}=C_{12} \epsilon_{x x}+C_{11} \epsilon_{y y} .
$$

Here $F_{y}$ is the restoring force applied on the surface area $A, C_{11}$ and $C_{12}$ are the in-plane elastic stiffness constants. Assuming the validity of Hooke's law, the elastic deformation energy $E_{\mathrm{D}}$ 


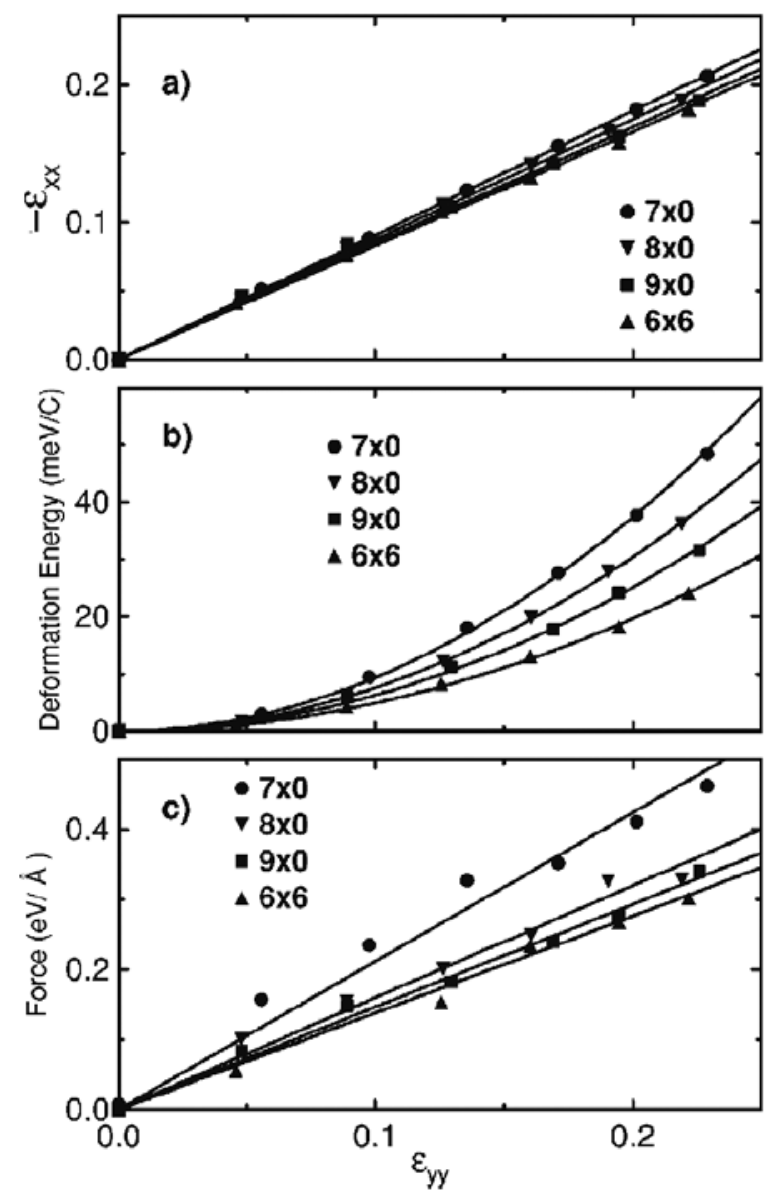

Figure 18. (a) The strain component $\epsilon_{x x}=\left(R_{0}-a\right) / R_{0}$ along the major axis as a function of the applied strain $\epsilon_{y y}=\left(R_{0}-b\right) / R_{0}$ along the minor axis. The slope is the in-plane Poisson ratio, $v_{\|}$. (b) Variation of the elastic deformation energy per carbon atom. (c) The restoring force on fixed carbon atoms. For the $(8,0)$ SWNT, the force is scaled by 0.5 since it is only on one carbon atom, while for the other tubes it is on two carbon atoms. (Reproduced from [43].)

(i.e. the difference between the total energies of radially deformed and undeformed SWNTs) is

$$
E_{\mathrm{D}}=\Omega\left[\frac{1}{2}\left(1+v_{\|}^{2}\right) C_{11}-v_{\|} C_{12}\right] \epsilon_{y y}^{2}
$$

in terms of the in-plane Poisson ratio, $v_{\|}[43]$ :

$$
v_{\|}=-\frac{\epsilon_{x x}}{\epsilon_{y y}}=\frac{C_{12}}{C_{11}} .
$$

Deformation energies obtained from classical elasticity in equation (8) are compared with those calculated from first principles in figure 18. Interestingly, the quadratic form obtained from classical theory fits very well to the elastic deformation energy calculated from first principles. Hooke's relation, and hence the elastic character of the deformations, persists up to $\epsilon_{y y}=0.25$. It is also noted that the SWNT becomes stiffer as $R$ decreases. The variation of the restoring forces is expected to be linear in the elastic range. The restoring forces in figure 18(c) are in overall agreement with this argument, except the deviations at certain data 

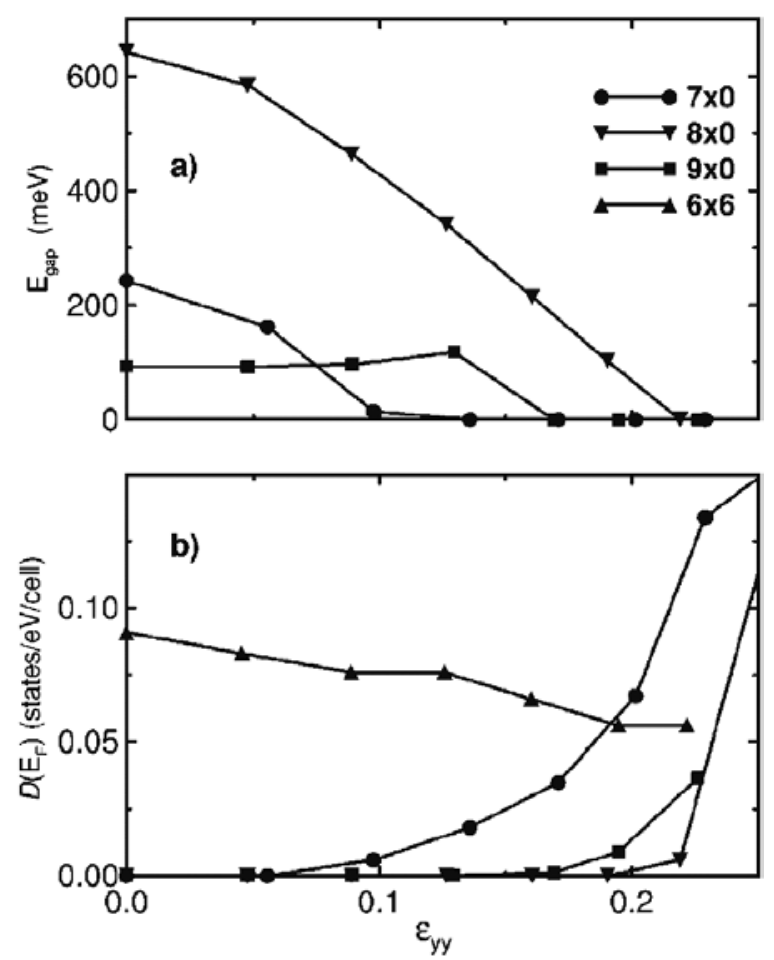

Figure 19. (a) The variation of the band gap, $E_{\mathrm{g}}$. (b) The density of states at the Fermi level $\mathcal{D}\left(E_{\mathrm{F}}\right)$ as a function of the applied strain $\epsilon_{y y}$. (Reproduced from [43].)

Table 4. In-plane elastic constants of SWNTs. All elastic constants are in GPa except for $v_{\|}$ which is unitless. $C_{\text {eff }}=C_{11}\left(1-v_{\|}^{2}\right)$. (Reproduced from [43].)

\begin{tabular}{lllrll}
\hline & Radius $(\AA)$ & \multicolumn{1}{l}{$v_{\|}$} & \multicolumn{1}{l}{$C_{\text {eff }}$} & \multicolumn{1}{l}{$C_{11}$} & \multicolumn{1}{l}{$C_{12}$} \\
\hline$(7,0)$ & 2.76 & 0.904 & 129.88 & 713.36 & 645.15 \\
$(8,0)$ & 3.14 & 0.874 & 98.70 & 416.88 & 364.20 \\
$(9,0)$ & 3.52 & 0.864 & 91.02 & 319.67 & 270.36 \\
$(6,6)$ & 4.06 & 0.828 & 86.12 & 273.46 & 226.34 \\
\hline
\end{tabular}

points due to uncertainties in the first-principles calculations, which are amplified because the force is a derivative quantity. Elastic constants calculated from first principles are listed in table 4 .

\subsection{Effect on the electronic structure}

First-principles calculations confirm that the band gaps of zigzag tubes reduce with applied strain and eventually vanish leading to metallization. Figure 19 summarizes the variation of the band gap and the density of states at the Fermi level, $\mathcal{D}\left(E_{\mathrm{F}}\right)$, as a function of the applied strain. For $(7,0)$ and $(8,0)$ SWNTs the band gaps decrease monotonically and the onset of an insulator-metal transition follows with the band closures occurring at different values of strain. Upon metallization, $\mathcal{D}\left(E_{\mathrm{F}}\right)$ increases with increasing strain. However, the behaviour of the $(9,0)$ tube is different. Initially, the band gap increases with increasing strain, but then 

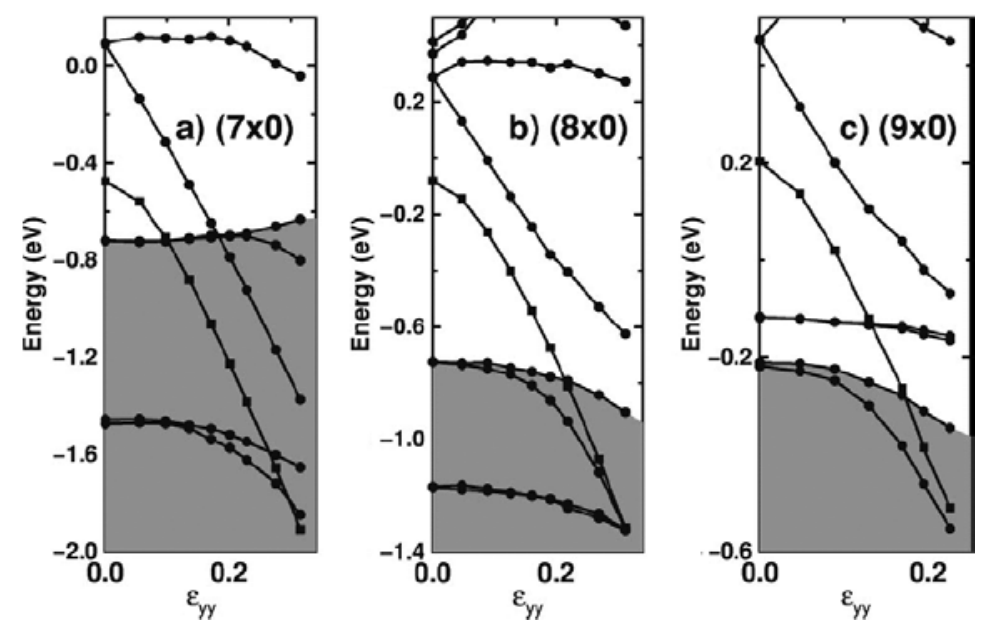

Figure 20. The variation of the energy eigenvalues of states near the band gap at the $\Gamma$ point of the BZ as a function of the applied strain. The shaded region is the valence band. The singlet state originating in the conduction band is indicated by squares. (Reproduced from [43].)

decreases with strain exceeding a certain threshold value and eventually diminishes. For all these zigzag SWNTs the band gap strongly depends on the magnitude of the deformation and $E_{\mathrm{g}}$ is closed at $13 \%, 22 \%$ and $17 \%$ strain for $(7,0),(8,0)$ and $(9,0)$ nanotubes, respectively, whereas the armchair $(6,6)$ SWNT, which is normally metallic, remained metallic with a slowly decreasing $\mathcal{D}\left(E_{\mathrm{F}}\right)$ even for significant radial deformation. Earlier, Delaney et al [85] showed that the $\pi^{*}$ conduction and $\pi$ valence bands of a $(10,10)$ tube, which normally cross at the Fermi level with quasi-linear dispersion, open a pseudogap in the range of $\sim 0.1 \mathrm{eV}$ at certain directions of the $\mathrm{BZ}$ perpendicular to the axis of the tube owing to tube-tube interactions in a rope. The opening of the gap is caused by the broken mirror symmetry. Lammert et al [120] showed the gapping by squashing $(20,20)$ and $(36,0)$ metallic tubes, since then circumferential regions are brought into close proximity. Uniaxial stress of a few kilobars can reversibly collapse a small radius tube inducing a $0.1 \mathrm{eV}$ gap, while the collapsed large radius tubes are stable. In the study of Park et al [44], the band gap of the $(5,5)$ tube was monotonically increasing, probably due to bilayer interactions, since the separation of the two nearest walls of the tube became comparable to the interlayer distance of graphite.

In order to explain the band gap variation of $(n, 0)$ tubes, the energies of a few bands near the band gap are plotted as a function of strain in figure 20. The singlet $\pi^{*}$ state in the conduction band shifts downwards in energy much faster than the other states do with increasing strain. This is due to the curvature increasing with increasing radial deformation. Since the singlet $\pi^{*}$ state lies below the doubly degenerate $\pi^{*}$ states for both $(7,0)$ and $(8,0)$ SWNTs, their band gaps are closed monotonically with increasing $\epsilon_{y y}$. On the other hand, for the $(9,0)$ SWNT this singlet $\pi^{*}$ state is above the doubly degenerate $\pi^{*}$ states. The increase of the band gap at the initial stages of radial deformation is connected with the relatively high rate of downward shift of the doubly degenerate $\pi$ valence band relative to the $\pi^{*}$ conduction band under low strains. Once the singlet $\pi^{*}$ band, which shows faster decrease with strain, crosses the doublet conduction band and enters the gap, the band gap begins to decrease with increasing strain. Applying a radial strain through the tip of an atomic force microscope and simultaneously measuring the conductance, Minot et al [121] confirmed that the band structure of carbon nanotubes can be altered as predicted in $[24,43,44]$ earlier. 


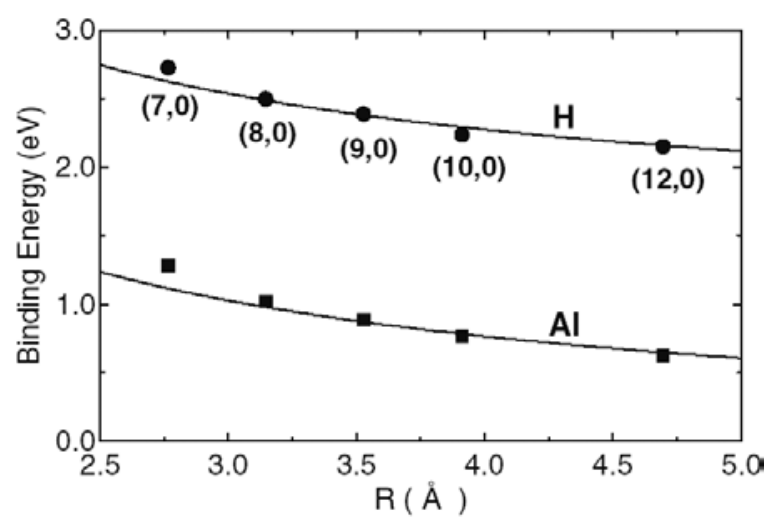

Figure 21. Binding energies $E_{\mathrm{b}}$ of single hydrogen and aluminium atoms adsorbed on the zigzag SWNTs versus the radius of the tube $R$. The solid curve is the fit to $E_{\mathrm{b}, \mathrm{A}}(R)=E_{0, \mathrm{~A}}+C_{\mathrm{A}} / R$ explained in the text. (Reproduced from [26].)

\subsection{Effect on the chemical reactivity}

Srivastava et al [58] examined the effect of conformational strain on the chemical reactivity of a SWNT. A $(10,10)$ SWNT, which was either bent or subjected to torsional strain, had kinks or ribbon structure on its surface. Actually the deformed places have sites with higher curvature as compared to the curvature of the bare $(10,10)$ SWNT. Using a many-body, bond order potential, they performed classical trajectory simulations on the interaction between the hydrogen atom and the strained nanotube. These predicted that the chemisorption of hydrogen atoms is enhanced by as much as $1.6 \mathrm{eV}$ in regions of high conformational strain. Analysis of atomic cohesive energies and the local density of states obtained from the empirical tight binding method showed that the sites with enhanced binding energies of hydrogen atoms correspond to destabilized cohesive energies and electronic states near the Fermi level. The heightened chemical reactivity of deformed sites was attributed to the introduction of radical p orbital character.

First-principles pseudopotential calculations have shown that the adsorption of foreign atoms on SWNTs can indeed be modified continuously and reversibly [26]. The effect of radial deformation becomes noticeable through the binding properties of foreign atoms on undeformed SWNTs. This situation is shown in figure 21, where the calculated binding energies $E_{\mathrm{b}}$ of $\mathrm{H}$ and $\mathrm{Al}$ adsorbed on the zigzag tubes are plotted as a function of the radius $R$ for $n=7,8,9,10,12$. As described in the previous sections, $\mathrm{H}$ is adsorbed at the top site, directly above the $\mathrm{C}$ atom of the tube; $\mathrm{Al}$ favours the $\mathrm{H}$ site. It is seen that $E_{\mathrm{b}}$ decreases with increasing radius (or decreasing curvature) and eventually saturates at a value corresponding to that for on graphene. The variation of the binding energies of $\mathrm{H}$ and $\mathrm{Al}$ with the radius of the zigzag tube fits to the curve given by the expression

$$
E_{\mathrm{b}, \mathrm{A}}(R)=E_{0, \mathrm{~A}}+\frac{C_{\mathrm{A}}}{R}
$$

where $E_{0, \mathrm{~A}}$ is the binding energy of the adatom $\mathrm{A}$ (here $\mathrm{H}$ or $\mathrm{Al}$ ) on the graphene plane and calculated to be 1.49 and $-0.02 \mathrm{eV}$ for $\mathrm{H}$ and $\mathrm{Al}$, respectively [26]. The fitting parameter, $C_{\mathrm{A}}$, is calculated to be $\sim 3.14 \mathrm{eV} \AA^{-1}$ for both $\mathrm{H}$ and Al. Calculated data for $n<7$ slightly deviate from the above simple $E_{\mathrm{b}}(R)$ relation, perhaps due to the fact that the singlet $\pi^{*}$ band, which is normally in the conduction band, falls into the band gap as a result of increased curvature. Note that, while the band gap shows significant change with $n$ (for example by going from 

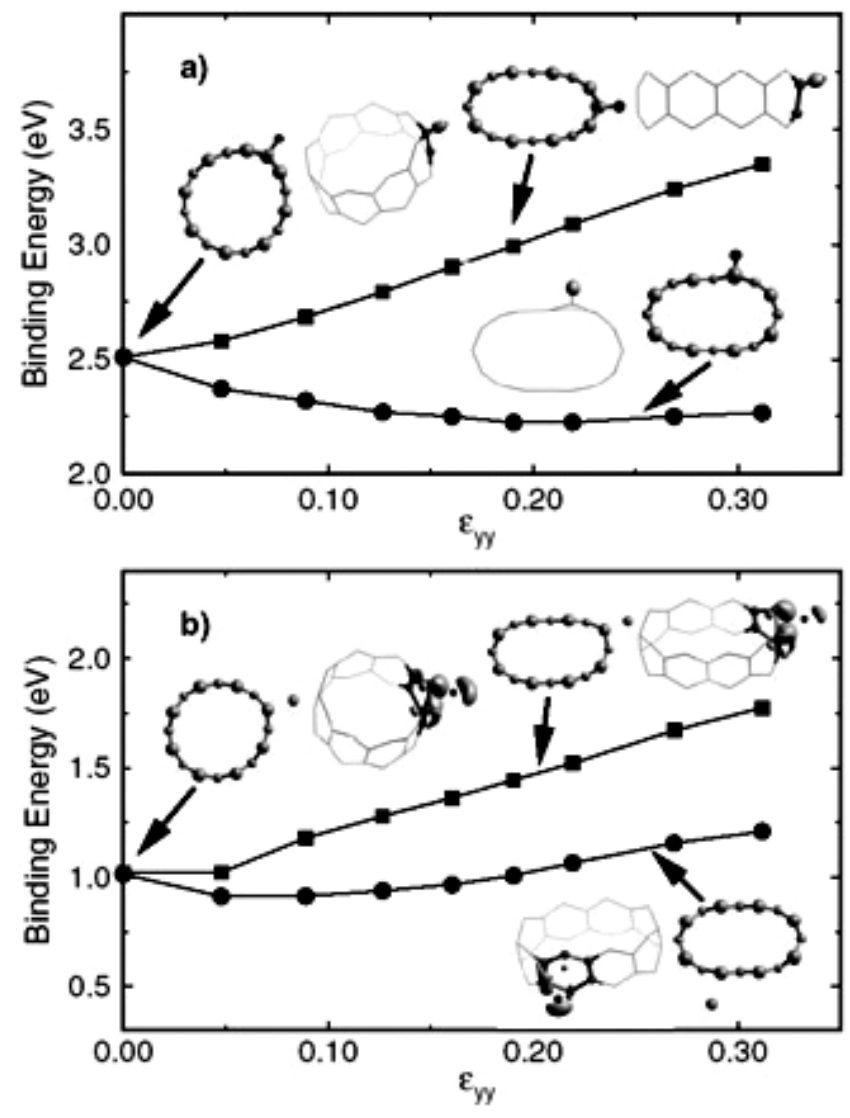

Figure 22. (a) Variation of the binding energies $E_{\mathrm{b}}$ of single hydrogen atoms adsorbed on a $(8,0)$ zigzag SWNT as a function of the radial deformation $\epsilon_{y y}$ defined in the text. The upper curve corresponds to $\mathrm{H}$ adsorbed on the high curvature site of the deformed tube. The lower curve is for the adsorption on the low curvature site. (b) As (a) but for a single Al atom. (Reproduced from [26].)

$(8,0)$ to $(9,0), E_{\mathrm{g}}$ changes from 0.65 to $\left.0.09 \mathrm{eV}\right)$, the binding energies vary smoothly with $R^{-1}$. Increase of $E_{\mathrm{b}}$ with decreasing $R$ (or with decreasing $n$ ) shows that for small $R$ the character of the surface deviates from that of the graphene. This result has been exploited to create different zones with different curvatures on the surface of a single SWNT by radial deformation and hence to attain different chemical reactivities.

Radial deformation can be created by applying a uniaxial compressive stress on a narrow strip on the surface of the SWNT [26, 43] where the circular cross section of the bare tube is distorted to an elliptical one with major and minor axes $a$ and $b$, respectively. In figure 22(a) the variation of the binding energies of a single hydrogen atom adsorbed on the $(8,0)$ surface shows that $E_{\mathrm{b}}$ at the sharp site, i.e. near one of the ends of the major axis, increases with increasing $\epsilon_{y y}$ (defined in equation (4)), and traces out the upper curve. The lower curve is associated with the adsorption on the flat site, i.e. near one of the ends of the minor axis. According to the predictions of this study, at the high curvature site, $E_{\mathrm{b}}$ has increased by $0.85 \mathrm{eV}$ for $\epsilon_{y y}=0.3$. On the other hand, $E_{\mathrm{b}}$ for the adsorption on the low curvature (flat) site first decreases with increasing strain and then saturates at an energy $0.25 \mathrm{eV}$ less than that corresponding to $\epsilon_{y y}=0$. The difference of binding energies of the sharp and flat sites 
is substantial and is equal to $\sim 1.1 \mathrm{eV}$ for $\epsilon_{y y}=0$. This is $44 \%$ of the binding energy of $\mathrm{H}$ on the undeformed SWNT. As a result of $\mathrm{H}$ adsorption, the $\mathrm{sp}^{2}$ character of the bonding of the tube has changed locally and become more like $\mathrm{sp}^{3}$. In figure 22(b) the binding energy of $\mathrm{Al}$ exhibits a behaviour similar to that of $\mathrm{H}$, despite $\mathrm{H}$ and $\mathrm{Al}$ favouring different sites on the $(8,0)$ tube; $E_{\mathrm{b}}$ at the sharp site of the deformed SWNT increases with increasing $\epsilon_{y y}$. For example $E_{\mathrm{b}}$ increases by $\sim 0.80 \mathrm{eV}$ for $\epsilon_{y y}=0.3$ which is $80 \%$ of the binding energy on the undeformed tube. For $\mathrm{Al}$ adsorbed on the flat site, $E_{\mathrm{b}}$ first decreases with increasing $\epsilon_{y y}$, then gradually increases. Adsorption of Al induces local changes in the atomic and electronic structures. For example, the surface of the tube where $\mathrm{Al}$ is adsorbed expands.

We see that according to the first-principles calculations the chemical reactivity of a zigzag SWNT can be modified reversibly and variably by radial deformation. The effects of deformation are significantly different for the zigzag and armchair SWNTs. It is remarkable that $\mathrm{Al}$, which does not bind to the graphite surface, can be adsorbed at the high curvature site of a zigzag tube under radial deformation with a significant binding energy. Similarly, it has been shown that a $\mathrm{H}_{2}$ molecule which is bound to the surface of $(8,0)$ becomes physisorbed at the high curvature site with a significant binding energy [130]. If the physisorbed $\mathrm{H}_{2}$ can approach the surface as closely as $1.5 \AA$, it dissociates into two $\mathrm{H}$ atoms, each of which becomes bound to adjacent $\mathrm{C}$ atoms of the SWNT at the surface. We believe that the tunable adsorption can have important implications for metal coverage and selective adsorption of foreign atoms and molecules on the carbon nanotubes and can lead to a wide variety of applications, ranging from hydrogen storage to chemical sensing, and new nanomaterials.

\subsection{Effect of pressure on nanoropes}

It is anticipated that physical properties can also be altered by intertube interactions between nanotubes packed in a hexagonal lattice, as so-called nanoropes. Intertube interactions in nanoropes can be probed by applying external pressure to vary the intertube distance. For fullerenes, such high pressure studies have yielded many interesting results [131] including new compounds such as the pressure induced polymeric phases of $\mathrm{C}_{60}$. Similar covalent bonding can occur between the nanotubes in a rope. Such a property could have important consequences for nanoscale device applications and composite materials that require strong mechanical properties, since nanoropes consisting of interlinked SWNTs will be significantly stronger than nanoropes composed of vdW packed nanoropes [122].

Raman studies on SWNT ropes have been carried out up to $25.9 \mathrm{GPa}$, and have indicated that the mode intensities and energies are not completely reversible upon pressure cycling [132]. These results have been interpreted as irreversible pressure induced changes in the atomic structure. Furthermore, observation of very large volume reduction and high compressibility [119] has suggested that a microscopic volume reducing deformation other than vdW compression can occur under high pressure. Some of these effects are tentatively attributed to crushing or flattening of nanotube surfaces in the rope, deforming the circular cross sections to elliptical or hexagonal ones [119]. Peters et al [133] reported that a structural phase transition occurred at $1.7 \mathrm{GPa}$. Furthermore they performed empirical uniform force field calculations and obtained equilibrium lattice constants of the $(10,10)$ nanotube rope as a function of applied pressure $P$. These calculations have indicated a sudden transition of structure at $1.75 \mathrm{GPa}$ [133]. Their interpretation of the structural transition has been objected to by Tang et al [134] on the grounds that their experiments were reversible up to $P=4 \mathrm{GPa}$.

Yildirim et al [123] were the first to investigate the effects of pressure on the ropes based on the first-principles calculations. The zigzag tubes $(5,0),(6,0),(7,0),(9,0)$ and the armchair tube $(6,6)$ were included in that study. The pressure dependence was determined 


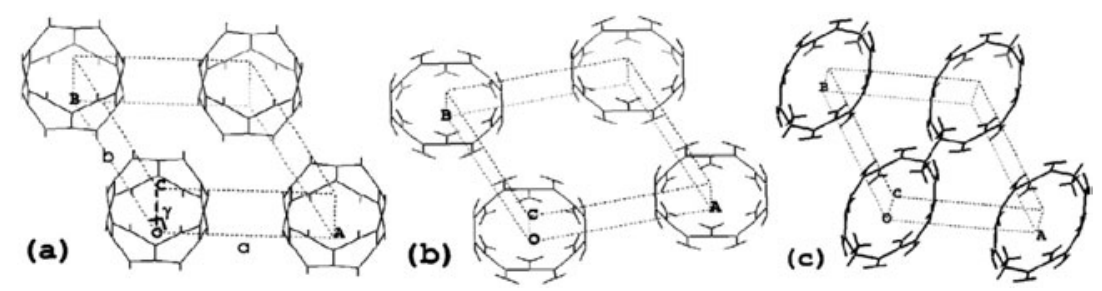

Figure 23. Optimized structures of SWNT ropes. (a) $(6,0)$ and (b) $(7,0)$ nanotubes are packed by $\mathrm{vdW}$ interaction under zero pressure and (c) $(7,0)$ one-dimensionally interlinked under pressure. The interlinked structure in (c) has lower total energy than the vdW packed structure in (b). Lattice parameters of the ropes, $a, b$ and $c$ and the $\gamma$ angle, are shown. (Reproduced from [123].)

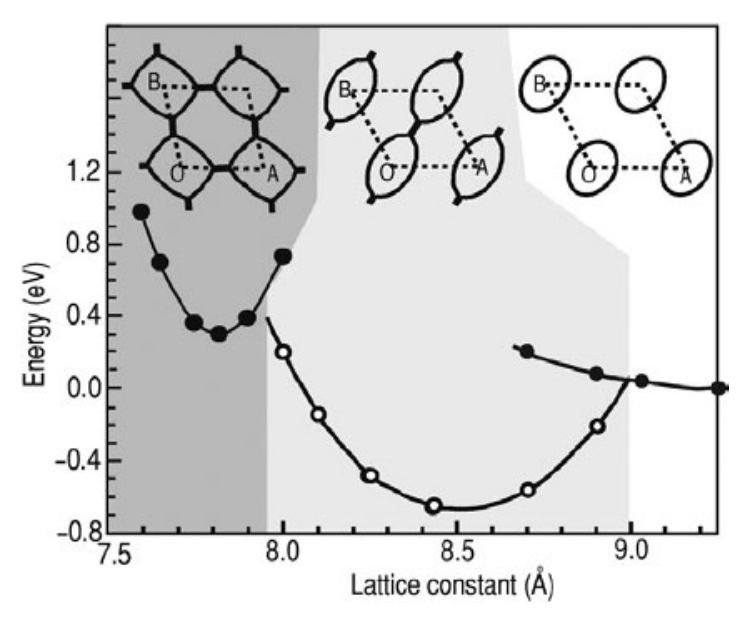

Figure 24. Total energy versus $2 \mathrm{D}$ lattice constant for $(7,0)$ nanotube ropes in different phases. The top view along the axis of the rope of the different phases is shown in the inset. The zero of energy is set to the total energy calculated for the optimized structure of the vdW packed rope at zero pressure. (Reproduced from [123].)

by minimizing the total energy as a function of nanotube separation, i.e. $E_{\mathrm{T}}(a, b)$, while the other parameters, including the atom position $c$ and $\gamma=2 \pi / n$, were optimized. At critical pressure, a structural phase transformation from a vdW lattice to a new orthorhombic (space group $C_{m} C_{m}$ ) lattice was observed in which the nanotubes were interlinked along the [110] direction. The covalent bonding between nanotubes occurred at the high curvature sites as a result of rehybridization of the carbon orbitals. Figure 23 compares the structure of a vdW packed $(7,0)$ nanorope with that of a 1D interlinked one. The same structural transformation was observed for the $(5,0),(6,0),(9,0)$ zigzag tubes.

The bonds interlinking two adjacent tubes have bond lengths comparable to those in diamond. This indicates that the rehybridization occurs from the $\mathrm{sp}^{2}$ to the $\mathrm{sp}^{3}$ type. However, the bond angles differ from the exact value of the tetrahedral angle and vary between $100^{\circ}$ and $120^{\circ}$ indicating some strain. For some $(n, 0)$ tubes the energy difference between the 1D interlinked structure and the vdW packed structure is small. Regardless of that, the 1D interlinked structure is stable once it is formed, since one has to overcome an energy barrier of $0.7 \mathrm{eV}$ in order to break the links, as illustrated in figure 24.

The total energies of the vdW and 1D interlinked phases cross each other at $a \sim 9 \AA$ with a small energy barrier of $46 \mathrm{meV} /$ unit cell $(552 \mathrm{~K})$ from the $\mathrm{vdW}$ side. The pressure 
for attaining this lattice constant is only about $0.3 \mathrm{GPa}$ for the vdW phase, indicating that polymerization of $\mathrm{vdW}(7,0)$ nanoropes could occur at modest pressures and temperatures. Conversely, $0.7 \mathrm{eV} /$ unit cell (or $25 \mathrm{meV} /$ atom) is required to go from the 1D interlinked phase to the vdW packed structure. This energy is comparable to the $1 \mathrm{D}$ polymerization energy of $\mathrm{C}_{60}$. Interestingly, at about $a \sim 8 \AA$ there occurs another interlinked phase of the $(7,0)$ nanorope, where nanotubes are interlinked along both $a$ and $b$ axes. This $2 \mathrm{D}$ interlinked phase is not favoured energetically. Its minimum lies $\sim 1 \mathrm{eV} /$ unit cell above the minimum of the 1D linked phase. However, once it is formed, it is stable and sixteen times stiffer $\left(\mathrm{d}^{2} E / \mathrm{d} a^{2}=13.7 \mathrm{eV} \AA^{-2}\right)$ than the vdW packed rope. The structures transform to even more complex structures at higher pressure. The ropes of the $(6,6)$ armchair tubes do not form interlinking up to $60 \mathrm{GPa}$. They are rather hexagonally distorted such that the local structure of nanotube faces is reminiscent of that of a graphite sheet. Upon releasing the pressure the hexagonally distorted (or polygonized) structure returns to the original one, indicating that the deformation of the $(6,6)$ nanorope is elastic (see figure 25 ). The above structural transformations induced by the applied pressure lead to significant changes in the electronic structure [123]. In spite of the fact that an individual $(7,0)$ tube is a semiconductor with $E_{\mathrm{g}}=0.65 \mathrm{eV}$, the dispersion of the bands near Fermi level in the direction perpendicular to the tube axis makes the vdW rope metallic. At high pressure $(30 \mathrm{GPa})$, the (2D interlinked) high density phase becomes a wide band gap insulator with $E_{\mathrm{g}} \sim 2 \mathrm{eV}$.

\section{Devices based on carbon nanotubes}

Much of the research on carbon nanotubes has been carried out with the motivation of generating devices, such as sensors, transistors and nanomagnets, and hence finding a feasible way to contribute to the objectives of miniaturization. Attempts to design and fabricate nanodevices from carbon nanotubes have exploited several properties discovered recently, some of them reviewed in the previous sections. In this section, we will first review recent progress made in calculating $I-V$ characteristics of nanostructures and present some of the device models or circuit elements, which are designed theoretically using SWNTs. Finally we will discuss the devices fabricated.

\subsection{Ab initio methods in transport calculations}

Accurate prediction of the transport properties of nanoscale atomic or molecular systems (nanotubes, in particular) including their current-voltage $(I-V)$ characteristics is essential for realization of a broad spectrum of device applications. Theoretical investigations of the transport properties of these nanoscale systems are based on calculations with various levels of sophistication, including phenomenological, semiclassical and quantum mechanical ab initio methods.

Semiempirical methods [20, 135-145] based on parametrized Hamiltonians, such as tight binding or extended Hückel models, have been widely used. Since these parametrized Hamiltonians are generally derived from the bulk or isolated molecular systems, the effects such as external bias and gate potential are usually not accounted for. Moreover, these models do not allow for structural relaxations to be performed. Semiempirical methods are not typically self-consistent; however, they are relatively simple and easy to implement. These methods provide some quantitative insight especially in cases where the charge transfer effects are relatively small. Some applications to carbon nanotubes include designation of a quantum dot by introducing pentagon-heptagon defects in a carbon nanotube [20], investigation of spin coherent quantum transport in carbon nanotube magnetic tunnel junctions [140], study 
(a) $(5,0)$
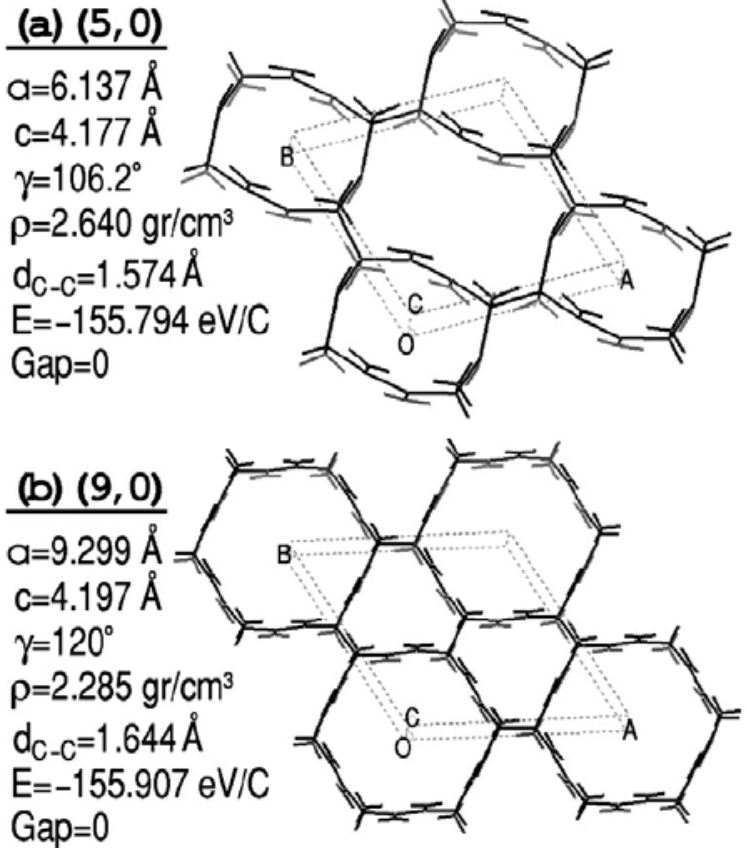

Gap $=0$

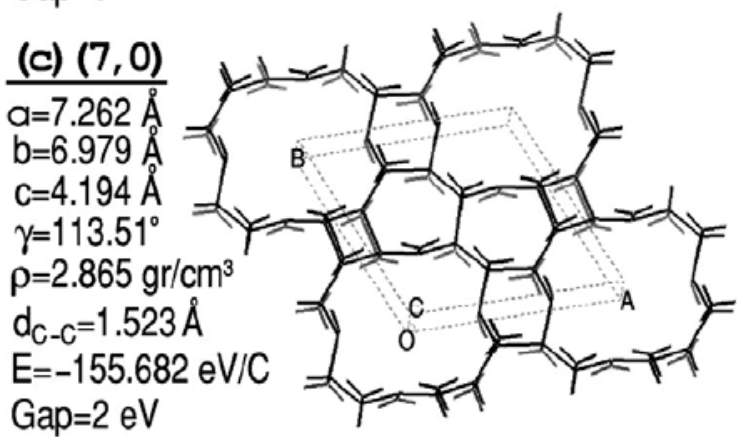

Gap $=2 \mathrm{eV}$

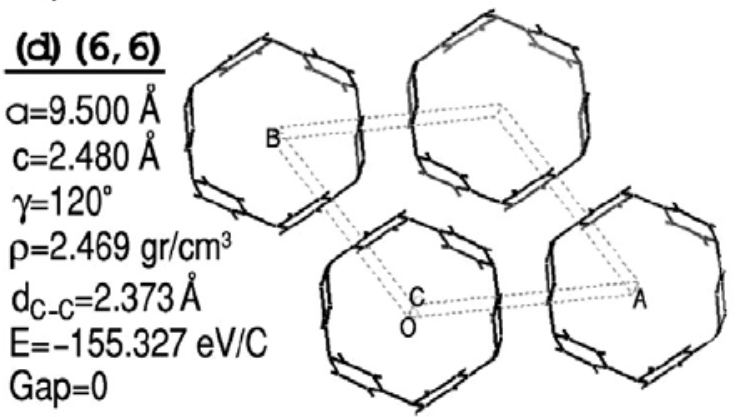

Figure 25. The two-dimensional crystal structure and relevant physical parameters for various high density phases of carbon nanotubes. (a) A $2 \mathrm{D}$ interlinked structure of $(5,0)$ nanotubes. (b) A hexagonal network of $(9,0)$ nanotubes, in which $(9,0)$ tubes are interlinked along $a, b$ and [110] directions. (c) A very dense structure of $(7,0)$ nanotubes obtained under $30 \mathrm{GPa}$. (d) The optimized structure of $(6,6)$ armchair tubes under $53 \mathrm{GPa}$. Nanotubes are distorted in such a fashion that the local nearest neighbour structure is reminiscent of graphite structure. $d_{\mathrm{C}-\mathrm{C}}$ indicates the smallest distance between two carbon atoms of nearest neighbour tubes in the rope. (Reproduced from [123].) 
a)

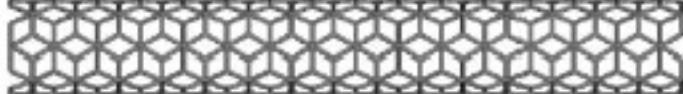

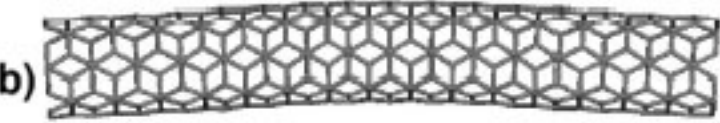

c)
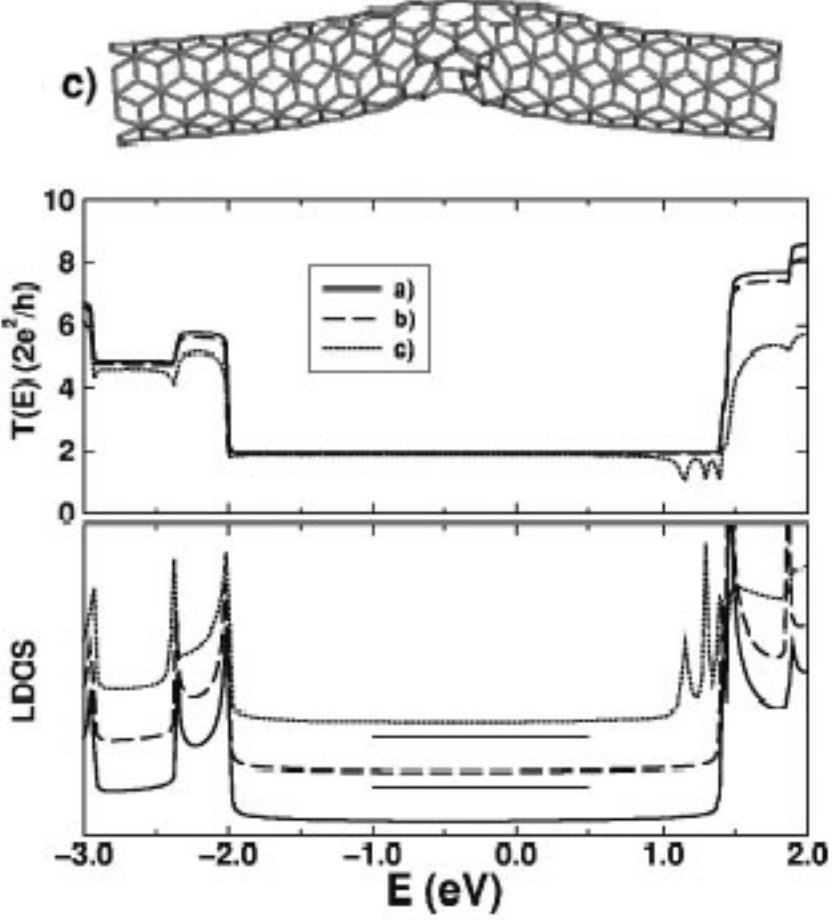

Figure 26. Upper panel: geometry of the bent $(4,4)$ nanotubes with bending angles (a) $\theta=0^{\circ}$, (b) $3^{\circ}$, (c) $6^{\circ}$. Lower panel: the calculated transmission function and LDOS for the corresponding geometries. (Reproduced from [144].)

of the dynamic conductance properties of carbon nanotubes of different helicities, both with and without defects [141], and calculation of transmission properties of extended [144] and finite size [145] carbon nanotubes. Electronic transport properties of extended nanotubes in the presence of distortions and mechanical deformations were studied by Nardelli [144] using the surface Green function matching formalism and a nearest neighbour $\pi$ orbital tight binding Hamiltonian [146]. The $\pi$ orbital bands were described via a single parameter, $V_{\mathrm{pp} \pi}=-2.75 \mathrm{eV}$. Calculations made for small radius $(4,4)$ nanotubes show that bending of the tube by small angles $\left(3^{\circ}\right.$ and $\left.6^{\circ}\right)$ does not drastically alter the basic electronic characteristics of the metallic $(4,4)$ tubes (see figure 26$)$.

A first-principles approach to the transport properties of nanoscale devices is based on supercell methods [147-154] where Kohn-Sham (KS) equations for the device are solved using periodic boundary conditions. The scattering states are then determined through a recursive technique considering the effective device potential between the perfect electrodes. The applicability of these methods is limited to the calculation of transport coefficients in the linear response regime, because the effective device potential is derived using periodic boundary 
conditions which cannot describe systems under an external bias or having nonidentical electrodes at the two ends. The method has been used to provide a possible explanation for the experimentally observed conductance of one quantum unit instead of two for nanotubes with one end dipped into a liquid metal such as mercury [153].

For nanoscale transport investigations, an alternative method, which was pioneered by Lang [155], is the open jellium approach [155-160] based on Lippman-Schwinger theory. Treating the leads in terms of a jellium model, the charge density of the open device is constructed from the scattering states. The KS equations are solved self-consistently with the appropriate boundary condition of the device under bias. The method, which allows $I-V$ curves to be calculated, is however limited by two major approximations. Constructing the charge density and the device potential using only scattering states is not enough for achieving a truly self-consistent solution. The omitted bound states, which exist within the device, must also be considered. Second, the real atomic structure of the electrodes is not represented in the jellium model.

In recent years, several first-principles methods have been developed to capture the basic properties of the challenging problem of nanoscale transport in the nonequilibrium regime [161-163]. These methods, essentially differing only in their numerical implementation, share most of the main ingredients of the treatment as they are imposed by the intrinsic properties of the problem at hand. Density functional theory (DFT) is employed to describe the atomistic nature of both the device and the electrodes self-consistently, including exchange-correlation and charging effects. Unlike conventional DFT simulation schemes, in order to account for the infinite and open nature of the system due to the electrodes, DFT is used in combination with the nonequilibrium Green function (NEGF) formalism. The NEGF formalism provides a means for representing the effects of infinite electrodes in the form of self-energy terms. Also the nonequilibrium charge density of the device under the biased conditions to be used in DFT can be constructed through the Keldysh nonequilibrium Green function. It should be noted that this formalism also allows for a computationally efficient evaluation of the contribution of the bound states to the charge density.

More specifically, a nanodevice coupled to two semi-infinite metallic electrodes can be considered to be divided into three parts. These are the left (l), right (r) and central (c) regions. The central (device) region must be chosen to extend far enough into the electrodes that the effective device potential can be matched at the boundaries with the bulk potential of the electrodes (see figure 27). The effective potential of the device is generated using the information on the charge distribution inside the central region $\rho_{\mathrm{c}}(\vec{r})$. The charge distribution is conveniently calculated from the density matrix $\hat{\rho}$, which can be constructed using the nonequilibrium Green function as

$$
\hat{\boldsymbol{\rho}}=-\frac{\mathrm{i}}{2 \pi} \int \mathrm{d} \varepsilon \mathbf{G}^{<}(\varepsilon)
$$

where

$$
\mathbf{G}^{<}(\varepsilon)=\mathbf{G}^{\mathrm{R}} \boldsymbol{\Sigma}^{<} \mathbf{G}^{\mathrm{A}}
$$

with $\mathbf{G}^{\mathrm{R}}$ and $\mathbf{G}^{\mathrm{A}}=\left(\mathbf{G}^{\mathrm{R}}\right)^{\dagger}$ denoting the retarded and advanced Green functions of the device (central region). $\boldsymbol{\Sigma}^{<}$corresponds to the injected charge from the electrodes. It can be expressed in terms of the Fermi distribution functions $f_{1}\left(\varepsilon-\mu_{1}\right)$ and $f_{\mathrm{r}}\left(\varepsilon-\mu_{\mathrm{r}}\right)$ of the left and right electrodes and their retarded self-energies $\Sigma_{1}^{\mathrm{R}}$ and $\Sigma_{\mathrm{r}}^{\mathrm{R}}$ as

$$
\Sigma^{<}=-2 \mathrm{i} \operatorname{Im}\left[f_{1} \Sigma_{1}^{\mathrm{R}}+f_{\mathrm{r}} \boldsymbol{\Sigma}_{\mathrm{r}}^{\mathrm{R}}\right] .
$$

Here $\mu_{1}$ and $\mu_{\mathrm{r}}$ are the Fermi levels deep in the left and right electrodes such that $V_{\mathrm{b}}=$ $\left(\mu_{1}-\mu_{\mathrm{r}}\right) / e$ is the external applied bias. The Green functions are evaluated by direct matrix 

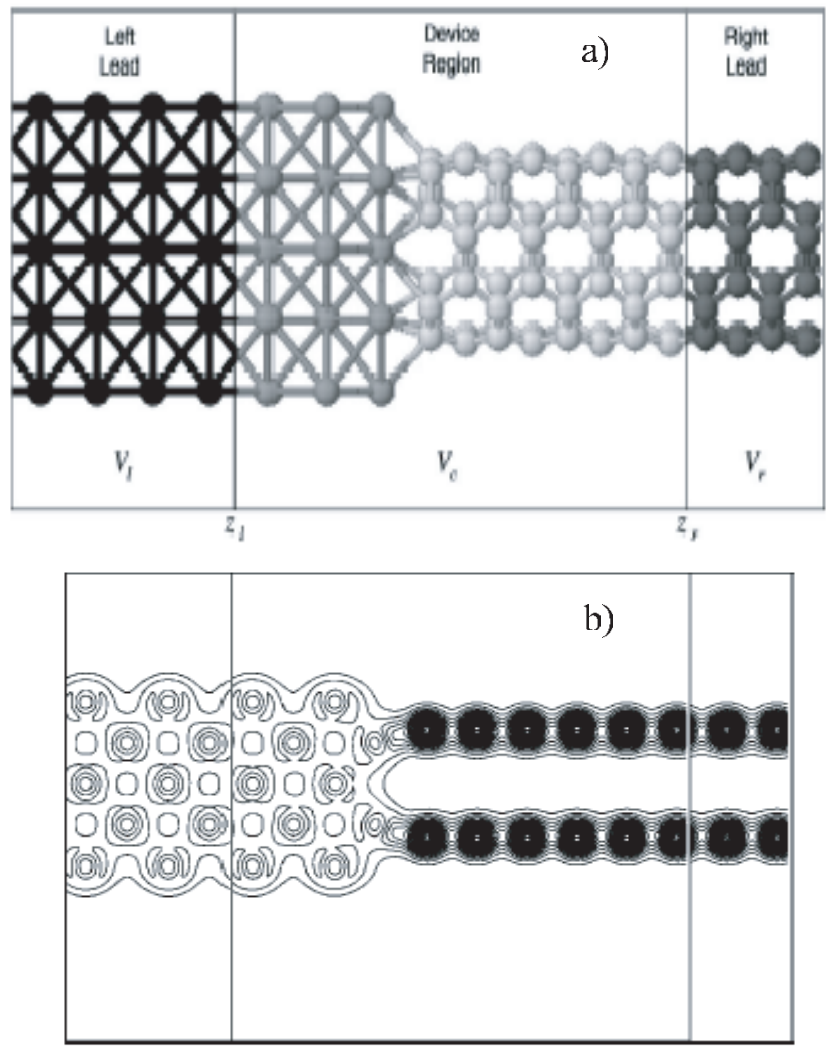

Figure 27. (a) The metallic electrode-nanotube interface. A semi-infinite $(4,4)$ armchair SWNT is in contact with a semi-infinite $\mathrm{Al}(100)$ metallic electrode. The device region is coupled to perfect atomic scale electrodes which extend to reservoirs at $z= \pm \infty$. (b) Charge density contours of the interface at equilibrium. The contours of the bulk lead match that of the device at the edges of the device region. (Reproduced from [161].)

inversions:

$$
\mathbf{G}^{\mathrm{R}}(\varepsilon)=\left[\varepsilon \mathbf{I}-\mathbf{H}-\boldsymbol{\Sigma}_{1}^{\mathrm{R}}-\boldsymbol{\Sigma}_{\mathrm{r}}^{\mathrm{R}}\right]^{-1}
$$

where $\mathbf{H}\left[\rho_{\mathrm{c}}(\vec{r})\right]$ is the Hamiltonian matrix of the central region within DFT and evaluated using the charge density obtained from equation (11). The cyclic procedure summarized above should be iterated until NEGF-DFT self-consistency is achieved.

The $I-V$ characteristics of the nanoscale device are calculated using the Landauer formula

$$
I\left(V_{\mathrm{b}}\right)=\frac{2 e}{h} \int \mathrm{d} \varepsilon T(\varepsilon)\left[f_{1}\left(\varepsilon-\mu_{1}\right)-f_{\mathrm{r}}\left(\varepsilon-\mu_{\mathrm{r}}\right)\right] .
$$

It should be noted that the transmission function $T(\varepsilon)$ depends both on temperature and on the applied bias and is expressed as

$$
T(\varepsilon)=\operatorname{Tr}\left[\boldsymbol{\Gamma}_{1}(\varepsilon) \mathbf{G}^{\mathrm{R}}(\varepsilon) \boldsymbol{\Gamma}_{\mathrm{r}}(\varepsilon) \mathbf{G}^{\mathrm{A}}(\varepsilon)\right]
$$

where $\boldsymbol{\Gamma}_{p}$ is the function describing the linewidth due to the coupling of the central device region to the electrode $p$. It is related to the corresponding self-energies of the electrodes in the form

$$
\Gamma_{p}=\mathrm{i}\left(\Sigma_{p}^{\mathrm{R}}-\Sigma_{p}^{\mathrm{A}}\right), \quad(p=1, \mathrm{r})
$$



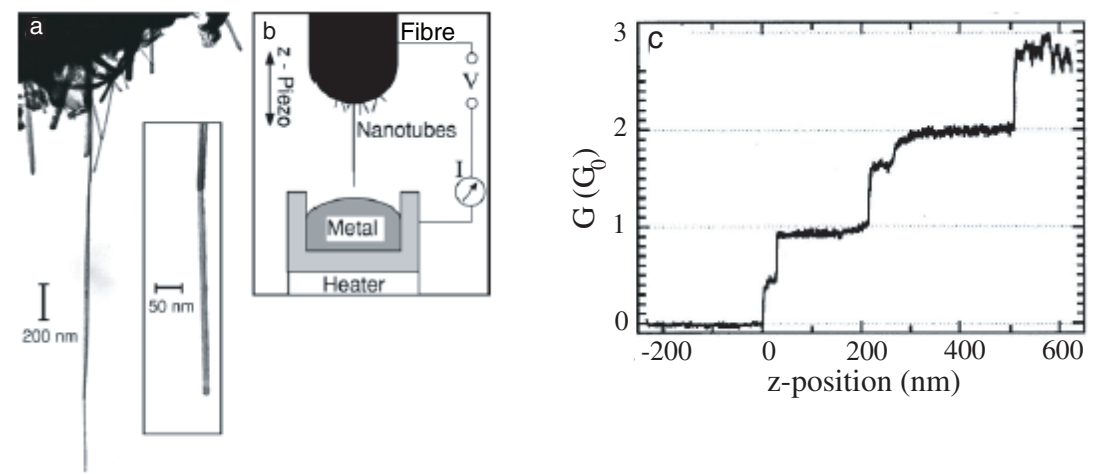

Figure 28. (a) A transmission electron micrograph (TEM) image of carbon nanotubes at the end of a nanotube fibre. (b) A schematic diagram of the experimental set-up. Nanotubes are lowered under SPM control into a liquid metal surface. (c) Variation of conductance with nanotube fibre position. Plateaus are observed corresponding to additional nanotubes coming into contact with the liquid metal. (Reproduced from [15].)

With the development of self-consistent ab initio methods based on DFT and the NEGF formalism, the transport properties of a broad set of nanoscale systems [164-168], including $\mathrm{C}_{60}$, linear chains of carbon, fullerene and silicon, and gold nanowires, are studied under nonequilibrium conditions. Some interesting features such as negative differential resistance in $I-V$ curves and the importance of the detailed atomic structure of the electrodes are reported.

Carbon nanotubes having different electronic structures depending on their chirality and radius are candidates for being main constituents of the future nanoelectronics technology. A considerable amount of experimental and theoretical effort has been devoted to investigating quantum transport properties of nanotubes. Calculations show that infinitely long, perfect, metallic single-wall carbon nanotubes have an equilibrium conductance of $G=2 G_{0}$, where $G_{0}=2 e^{2} / h$, due to two subbands crossing at the Fermi level $[11,12]$. The existence of pentagon-heptagon pair defects in infinite nanotubes is shown to alter the conductance pattern slightly [152]. The discrepancy between the theoretical calculations and the much smaller values of the nanotube conductance measured in experiments is attributed to poor nanotubeelectrode contacts.

In an interesting transport experiment performed by Frank et al [15] the conductance of MWNTs, dipped into a liquid metal to minimize the contact resistance effects, were found to be close to $G_{0}$, half the expected value. Figures 28 (a) and (b) show the transmission electron micrograph (TEM) image of the nanotubes used and the experimental set-up. The conductance is seen to increase as the nanotube fibre is dipped into the liquid metal in steps of $G_{0}$ as shown in figure 28(c). Each step corresponds to an additional nanotube coming into contact with the metal. Although narrower, two more steps at fractional $G_{0}$ values are also observed.

An explanation of the measured conductance pattern is provided by Choi and coworkers [153] in their ab initio calculation of the conductance of the $(10,10)$ SWNT with one end immersed in a jellium metal. They showed that of the two available channels, it is mostly $\pi$ band incident electrons that suffer strong back-scattering at the nanotube-metal contact, while the $\pi^{\star}$ band is responsible for the observed conductance of the system, i.e. $G=G_{0}$. In another account, using a semiempirical scattering technique Sanvito et al [169] calculated the conductance of a MWNT with a special geometry and provided a possible explanation of the measured [15] fractional conductance values in terms of interwall interactions in a MWNT. Later, Taylor et al [161] simulated transport properties of a SWNT-metal interface using the 
NEGF-DFT formalism and a semi-infinite Al electrode of finite cross section (see figure 27). They showed that there are two transmission eigenvectors which connect the two sides of the electrode-nanotube interface. The two transmission eigenvalues $T_{1}$ and $T_{2}$ were smaller than unity; however, their sum at the Fermi energy gave an equilibrium conductance almost equal to $G_{0}$ for a range of distances between the nanotube and the electrode. This result is consistent with both the nanotube-jellium calculation [153] and the experiment [15]. In a recent study Palacios et al [170] addressed the problem of conductance of finite length carbon nanotubes with realistic metal electrodes using a first-principles method called the Gaussian embedded cluster method [163]. They observed resonance patterns around the Fermi level in the conductance profiles. As electrodes, elemental $\mathrm{Ti}$ was found to make better high transparency contacts to carbon nanotubes compared to more traditional metals such as $\mathrm{Al}$ and $\mathrm{Au}$.

The electronic and transport properties of carbon nanotubes can be modified by doping with foreign atoms. The effects of substitutional nitrogen doping in carbon nanotubes under nonequilibrium conditions were investigated by Kaun et al [171] using NEGF-DFT formalism. Nitrogen doping of nanotubes does not cause substitutional structural relaxation; however, the step-like equilibrium conductance pattern of the nanotube is altered due to the action of a substantial number of nitrogen atoms as elastic scattering centres. It was found that for doped zigzag nanotubes even a single nitrogen atom substitution increases the current flow and for small radii tubes narrows the current gap. Periodic doping of nitrogen atoms in a semiconducting nanotube makes the structure metallic with a nonlinear $I-V$ characteristic and a negative differential resistance at higher biases. It is interesting that breaking of symmetry by the impurities can raise the current flow in semiconducting tubes and reduce the current slightly in metallic ones. All these nonequilibrium transport properties can be understood from the simple physical picture of alignment and misalignment of current carrying bands of the device due to applied bias.

\subsection{Device models}

Several novel properties of carbon nanotubes, which have been revealed through the active experimental and theoretical research during the last decade, have been combined in nanodevice operation or circuit elements. These are semiconductor heterostructures or metalsemiconductor junctions; resonant tunnelling double-barrier (RTDB) and multiple-quantumwell structures (MQWS); metallic nanorings for persistent currents and solenoids; metalnanotube contacts and Schottky barriers; interconnects; etc.

SWNT-SWNT junctions. Structural defects can affect the atomic and electronic structure of nanotubes. The role of topological defects, such as 5/7 pentagon-heptagon defects, has been investigated by different groups. Charlier et al [19] carried out an extensive study on the topological 5/7 defects in different directions on the surface of the $(12,0)$ SWNT and revealed their effects on the atomic and electronic structure by using a recursion method within the tight binding description of the carbon $\pi$ bands. They found that the $5 / 7$ defect can induce changes in diameter and chirality of the nanotube. For example, if the 5/7 defect can be aligned exactly on the axis of the $(n, 0)$ zigzag tube, the chirality remains the same, but the diameter becomes smaller. If the $5 / 7$ defect is perpendicular to the axis of the tube, the diameter is conserved, but the zigzag SWNT becomes chiral. Chirality increases with increasing number of 5/7 defects perpendicular to the axis of the tube. The 5/7 defects in any orientation on the surface of SWNTs change both the diameter and the chirality. Not only atomic or geometric structure, but also the electronic structure around the Fermi level gets 

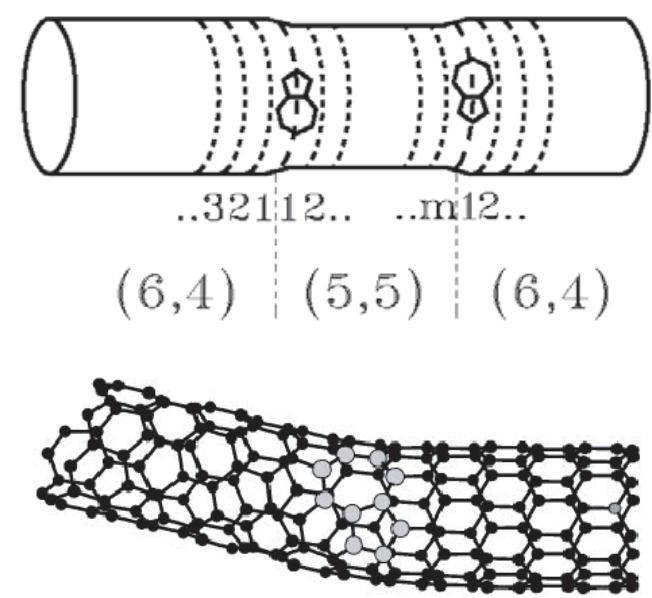

Figure 29. Energies of the discrete states of the $(6,4) / m(5,5) /(6,4)$ quantum dots as a function of the number of $(5,5)$ unit cells $m$. (Reproduced from $[20,173]$.)

modified by the presence of $5 / 7$ defects. Most importantly, nanotubes with different chiralities and different electronic properties and diameters can be connected using such a pair defect to form different kinds of heterostructures. These heterostructures (such as metal-semiconductor and semiconductor-semiconductor) can form rectifiers or diodes and hence they can play an important role in nanoelectronics. In fact, the calculation of electronic transport through such junctions yielded tunnelling conductance and hence indicated that they can be used as building blocks of nanoscale electronic devices [172]. Chico et al [173] studied the electronic structure of $(8,0)-(7,1)$ and $(8,0)-(5,3)$ junctions, which were formed from a single $(8,0)$ SWNT by introducing a single and triple $5 / 7$ defect pair, respectively. For the case of the $(8,0)-$ $(7,1)$ junction, it corresponds to a heterostructure between a semiconductor having a band gap of $0.62 \mathrm{eV}$ and a semimetal. The second junction, $(8,0)-(5,3)$, is a semiconductorsemiconductor heterostructure with two different band gaps.

It is clear that by the controlled generation of 5/7 defects on a chiral SWNT one can form a variety of SWNT-SWNT junctions with different heterostructure characteristics. Chico et al [20] went further and proposed an interesting heterostructure, namely a new type of quantum dot based solely on single SWNTs. It is made from a series of $(6,4) / m(5,5) /(6,4)$ SWNT junctions. Here the $(6,4)$ tube is a semiconductor; $m(5,5)$ indicates $m$ unit cells of a metallic armchair tube. The $(6,4)$ tube was connected to the $(5,5)$ tube smoothly, introducing heptagon-pentagon defects. In this way formation of undesirable interface states has been eliminated. Normally, a metallic nanostructure between two semiconducting ones forms a quantum dot and this has quantized electronic states. Using the empirical tight binding method and Green function matching approach along with the transfer matrix technique, Chico et al [20] showed that electronic states in the metallic $m(5,5)$ part are indeed confined and exhibit a sharp, $\delta$ function-like density of states. Moreover, their level spacings vary depending on the value of $m$, showing a clear quantum size effect (see figure 29).

Quantum structures generated by radial deformation. The metallization of semiconducting SWNTs under radial deformation (discussed in section 6) has been proposed to generate quantum structures $[24,44,118]$. The idea is simple and originates from the numerous works done on semiconductor heterostructures $\left(\mathrm{A}_{n} \mathrm{~B}_{m}\right)$ and quantum dots [174]. It is known that 
owing to the band offsets of the semiconductor heterostructures, the energies of the band states of one semiconductor B may fall into the band gap of the adjacent semiconductor A. According to the effective mass approximation, the height (depth) of the conduction (valence) band edge of A measured from that of $\mathrm{B}, \Delta E_{\mathrm{C}}\left(\Delta E_{\mathrm{V}}\right)$, behaves as a potential barrier for electrons (holes). For example, $m$ layers of B between $n$ layers of two As form a quantum well yielding confined electronic states. The depth of the well and the width of the barrier and well (in terms of number of layers $n$ and $m$, respectively) are crucial parameters for monitoring the resulting electronic properties. By repeated formation of $\left(\mathrm{A}_{n} \mathrm{~B}_{m}\right)$ one generates MQWSs or RTDBs by combination $\left(\mathrm{A}_{n} \mathrm{~B}_{m} \mathrm{~A}_{n}\right)$. Similar to what one achieves in semiconductor heterostructures, periodic MWQSs or finite RTDB structures can be realized on an individual s-SWNT and their electronic properties can be variably and reversibly monitored.

Performing empirical tight binding and also first-principles calculations, Kiliç et al [24] have shown that a MQWS is generated by applying different radial deformations at adjacent parts of a $(7,0)$ s-SWNT and repeating them periodically. They considered a supercell consisting of 16 unit cells of the $(7,0)$ SWNT; $n$ of the unit cells were kept undeformed and the remaining $m=16-n$ were radially deformed. Tight binding charge densities of the band edge states for superlattices corresponding to $n=4,8,12$ have clearly demonstrated the confinement of states and quantum well structure formation. Later, the strain induced quantum well structures were treated by means of SCF first-principles calculations on a superlattice consisting of $12(8,0)$ SWNT unit cells [18]; half of them were radially strained with $\epsilon_{y y}=0.16$; the remaining half were left undeformed-i.e. $\epsilon_{y y}=0$. Within the superlattice geometry, the adjacent unstrained and strained parts were repeated yielding a periodic band gap modulation. Applying the standard method for band line-up explained in detail below [175], using a SCF crystal potential and referring to the energies at the top of the valence band, the misfit of the valence band edges between adjacent (i.e. undeformed and deformed) zones has been found to be $\sim 180 \mathrm{meV}$. This indicates a staggered band line-up. As in the empirical tight binding calculations, it has been demonstrated that quantum structures can be formed on a single SWNT. Tombler et al [118] have shown that a quantum dot can be produced by bending (and hence inducing radial deformation of) the SWNT at a given point.

Quantum structure formation by modulating hydrogenation. A model of an electronic device based on a similar band gap modulation and hence MQWS formation has been proposed recently [176]. In this case the band gap modulation is provided by the modulating hydrogen adsorption (see figure 30). The localization of states in one of the zones (either a $\left(\mathrm{C}_{32}\right)_{l}$ (i.e. specified by $l$ ) zone or a $\left(\mathrm{C}_{32} \mathrm{H}_{32}\right)_{q}$ (i.e. specified by $q$ ) zone) is based on the physical grounds discussed above.

Formation of different zones leading to different electronic structures on the same $(8,0)$ SWNT upon modulating the $\mathrm{H}$ adsorption, and also the effect of the superlattice size (i.e. $l, q$ and $l+q$ ), is further analysed by examining the electronic potential [176]. The electronic potential which consists of Hartree, exchange and ionic pseudopotentials, $V_{\mathrm{e}}(\mathbf{r})=V_{\mathrm{H}}(\mathbf{r})+V_{x}(\mathbf{r})+V_{\text {ion }}(\mathbf{r})$, is calculated self-consistently. Figure 31 shows the planar averaged electronic potential

$$
\bar{V}_{\mathrm{e}}(z)=\frac{1}{a_{\mathrm{s}} b_{\mathrm{s}}} \int V_{\mathrm{e}}(x, y, z) \mathrm{d} x \mathrm{~d} y
$$

and the running average of the planar averaged potential, $\bar{V}_{\mathrm{r}, \mathrm{e}}(z)$, along the axis of the SWNT. Here $a_{\mathrm{s}}$ and $b_{\mathrm{s}}$ are superlattice parameters. As seen in figure 31(a), the short periodicity, $l=q=1$, is not sufficient to induce two adjacent zones with markedly different electronic structures. In figure 31(b) the differences between two zones become pronounced and $\Delta V$ 

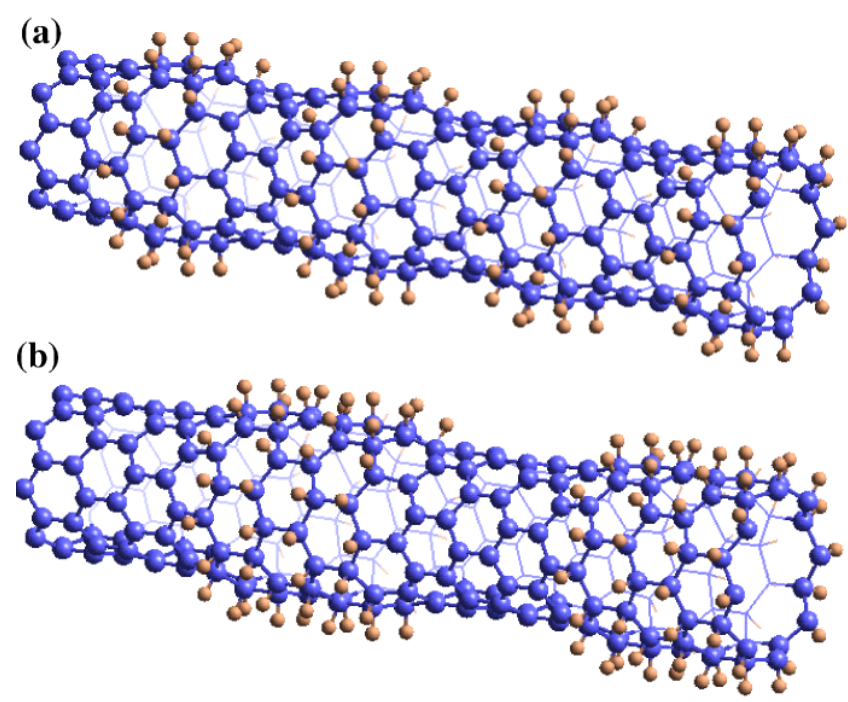

Figure 30. Superlattice structures, $\left[\left(\mathrm{C}_{32}\right)_{l}\left(\mathrm{C}_{32} \mathrm{H}_{32}\right)_{q}\right]$ formed on a single $(8,0)$ SWNT by modulating adsorption of hydrogen atoms. (a) $l=q=1$; (b) $l=q=2$. The positions of $64 \mathrm{H}$ and $128 \mathrm{C}$ atoms, as well as the superlattice parameter $c_{\mathrm{s}}$, are calculated from full structure optimization by using conjugate gradient method. (Reproduced from [176].)

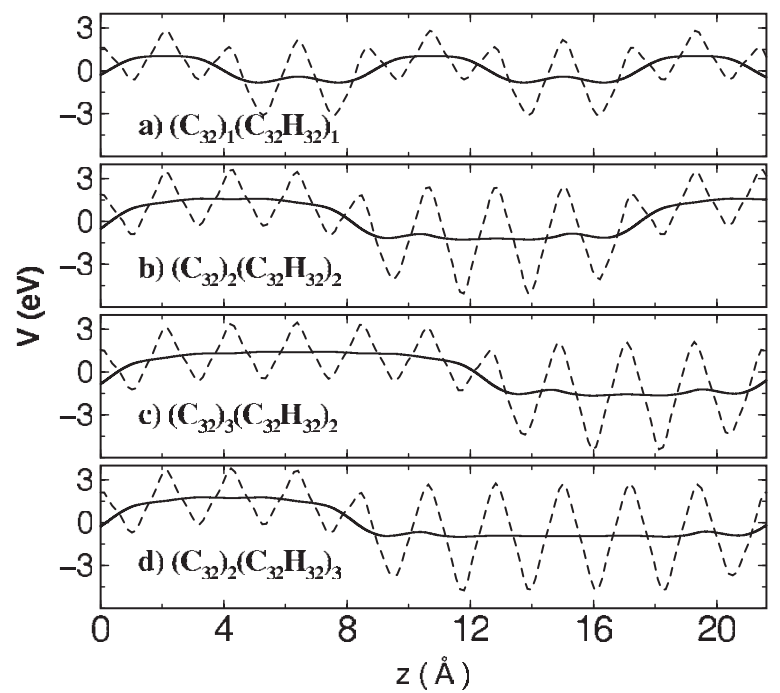

Figure 31. Averages of calculated self-consistent electronic potentials of the superlattices. (a) $\left[\left(\mathrm{C}_{32}\right)_{1}\left(\mathrm{C}_{32} \mathrm{H}_{32}\right)_{1}\right]$; (b) $\left[\left(\mathrm{C}_{32}\right)_{2}\left(\mathrm{C}_{32} \mathrm{H}_{32}\right)_{2}\right] ;$; c) $\left[\left(\mathrm{C}_{32}\right)_{3}\left(\mathrm{C}_{32} \mathrm{H}_{32}\right)_{2}\right] ;$ (d) $\left[\left(\mathrm{C}_{32}\right)_{2}\left(\mathrm{C}_{32} \mathrm{H}_{32}\right)_{3}\right]$. Dashed and thick curves are planar averaged, $\bar{V}_{\mathrm{e}}(z)$, and running averaged, $\bar{V}_{\mathrm{r}, \mathrm{e}}(z)$, respectively. Relatively high cell averaged potential zones correspond to $\left(C_{32}\right)_{l}$. See the text. (Reproduced from [176].)

increases for the superlattice; $l=q=2$. Figures 31(c) and (d) indicate that the potential, $\bar{V}_{\mathrm{r}, \mathrm{e}}(z)$, becomes flatter as $l$ and $q$ increase from 2 to 3 and two adjacent zones display markedly different electronic structures.

Band line-up of zones, $\left(\mathrm{C}_{32}\right)_{l}$ and $\left(\mathrm{C}_{32} \mathrm{H}_{32}\right)_{q}$, is a complex process involving the relaxation of the atomic structure and charge transfer at the interfaces and also resulting in modification 


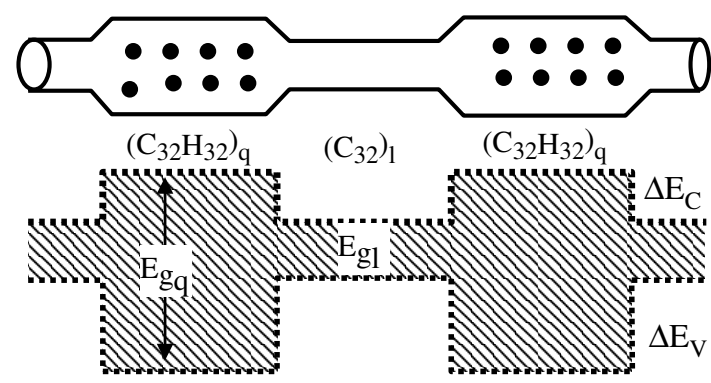

Figure 32. A schematic illustration of the energy band diagram and band offsets estimated by using averaged self-consistent electronic potentials, $\bar{V}_{\mathrm{r}, \mathrm{e}}(z)$, calculated for the $l$ and $q$ zones, $\Delta E_{B, l(q)}$ and band gaps, $E_{\mathrm{g} 1}$ and $E_{\mathrm{g} 2}$, calculated for $l=\infty$ and $q=\infty$. The band diagram indicates a type-I band line-up appropriate for a resonant tunnelling double-barrier structure. (Reproduced from [176].)

of the electronic potential. Experimental and theoretical methods have been proposed in the past to determine the band offsets and hence to obtain the band diagram perpetuating along the semiconductor heterostructures. A solution of this problem [175] involves the following steps. First, the average electronic potentials corresponding to $\bar{V}_{\mathrm{r}, \mathrm{e}}(z)$ for finite $l$ and $q$, as well as for infinite $l$ and $q,\left(\mathrm{C}_{32}\right)_{l=\infty}$ and $\left(\mathrm{C}_{32} \mathrm{H}_{32}\right)_{q=\infty}$, are calculated. The actual atomic positions, especially those at the interface, and the charge transfer between regions are of crucial importance for the band offsets and are incorporated self-consistently in the calculated average electronic potential. Secondly, for the case of $l=q=\infty$ the energy difference between the edge of the valence band $E_{\mathrm{V}}$ and the average potential, i.e. $\left.\Delta E_{\mathrm{B}}\right|_{l(q)=\infty}=E_{\mathrm{V}, l(q)=\infty}-\left.\bar{V}_{\mathrm{r}, \mathrm{e}}\right|_{l(q)=\infty}$, are calculated. $\Delta E_{\mathrm{B}}$ for $\left(\mathrm{C}_{32}\right)_{l=\infty}$ and $\left(\mathrm{C}_{32} \mathrm{H}_{32}\right)_{q=\infty}$ is found to be 2.62 and $3.91 \mathrm{eV}$, respectively. In the third step, one assumes that $\Delta E_{\mathrm{B}}$ is unchanged for finite $l$ and $q$ and determines the valence band edges of a particular superlattice with given $l$ and $q$ by adding $\left.\Delta E_{\mathrm{B}}\right|_{l(q)=\infty}$ to the corresponding average potential values $\bar{V}_{\mathrm{r}, \mathrm{e}}$ of the $l$ and $q$ zones. At the end, the energies of the valence band edges of two adjacent zones (i.e. hydrogen-free and exohydrogenated) are fixed. Then, the valence band offset of the superlattice is $\Delta E_{\mathrm{V}}=E_{\mathrm{V}, l}-E_{\mathrm{V}, q}$. The energies of the conduction band edges of the superlattice are determined by adding band gaps of different zones (calculated for $l=\infty$ and $q=\infty$ ) to the energies of the valence band edges, i.e. $E_{\mathrm{C}, l}=E_{\mathrm{V}, l}+E_{\mathrm{g} 1}$ and $E_{\mathrm{C}, q}=E_{\mathrm{V}, q}+E_{\mathrm{g} 2}$. Here, $E_{\mathrm{g} 1}$ and $E_{\mathrm{g} 2}$ are the band gaps of hydrogenated and hydrogen-free zones, respectively. The conduction band offset is then determined by subtracting these latter energies, i.e. $\Delta E_{\mathrm{C}}=E_{\mathrm{C}, q}-E_{\mathrm{C}, l}$. Figure 32 presents a schematic illustration of the band diagram obtained in [176].

The present discussion for the periodically repeating quantum wells can be extended to finite systems, for example to the resonant tunnelling double barriers, made by a zone $\left(\mathrm{C}_{32}\right)_{l}$ placed between two zones of $\left(\mathrm{C}_{32} \mathrm{H}_{32}\right)_{q}$, i.e. $\left(\mathrm{C}_{32} \mathrm{H}_{32}\right)_{q}\left(\mathrm{C}_{32}\right)_{l}\left(\mathrm{C}_{32} \mathrm{H}_{32}\right)_{q}$. Metallic reservoirs adjacent to both barriers, i.e. $\left(\mathrm{C}_{32} \mathrm{H}_{32}\right)_{q}$, and metallic connects of the device can be achieved by metal coating $[102,103,109]$. Also a metal-semiconductor heterostructure can be formed by modulating the hydrogenation of an $(n, n)$ armchair SWNT. In this way metallic connects are provided by the hydrogen-free ends of the tube.

SWNT-metal contact. The switching of the current in a s-SWNT at room temperature by an external electric field has been utilized to fabricate a new field effect transistor (FET) [99-101]. The interaction between the s-SWNT and metal electrode and the Schottky barrier, $\Phi_{\mathrm{B}}$, formed 


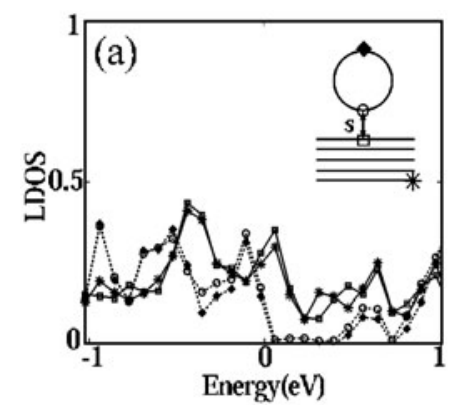

(d)

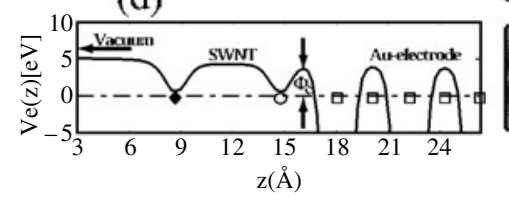

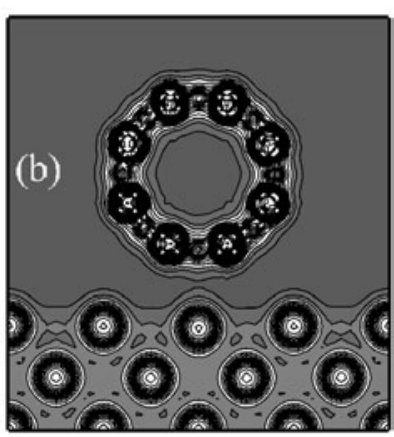

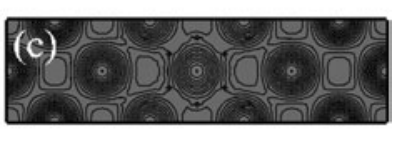

Figure 33. (a) Local densities of states (LDOSs) at different $\mathrm{Au}$ and $\mathrm{C}$ atoms of the $(8,0)$ zigzag SWNT side-bonded to the $\mathrm{Au}(100)$ surface. The locations of atoms are shown in the inset. The zero of energy is taken at the Fermi energy $E_{\mathrm{F}}$. State densities shown by empty circles and filled diamonds correspond to six neighbouring carbon atoms. Other densities are for single atoms. (b) Contour plots of the SCF electronic potential, $V_{\mathrm{e}}(\mathbf{r})$, on a vertical plane. (c) As (b) but on a horizontal plane bisecting $\mathbf{s}$. In the dark grey regions, $V_{\mathrm{e}}(\mathbf{r})>E_{\mathrm{F}}$. (d) The variation of $V_{\mathrm{e}}(\mathrm{z})$ on a perpendicular line passing through the centre of the SWNT. (Reproduced from [179].)

thereof have been proposed as the origin of the FET operation. Model calculations have been carried out to provide further understanding of experimental $I-V$ characteristics $[177,178]$.

The origin of the potential barrier formed between an s-SWNT and a specific metal electrode has been investigated recently by using first-principles calculations [179]. The interaction and energy of binding between an individual Au atom and $(8,0)$ tube is essential for the contact. The contact on the gold electrode is formed by placing the s-SWNT on the Au(100) surface, so a surface Au atom faces the centre of the hexagon. The Au-SWNT equilibrium distance, $\mathbf{s}$, occurred at $3.18 \AA$ according to full structure optimization. This implies a weak $\mathrm{Au}-\mathrm{SWNT}$ interaction which is characterized by physisorption. The LDOSs calculated for two $\mathrm{Au}$ atoms at different sites (one is at the contact just below the SWNT hexagon; the second being furthest from the SWNT mimics the clean Au surface) in figure 33(a) have finite state density at the Fermi level. These two LDOSs are similar; there are only minute changes, whereas the LDOS of the carbon atoms at the contact still has a band gap. The Fermi level lies near the top of the valence band of the SWNT and is consistent with a small $\Phi_{\mathrm{B}}$ in the hole doping picture. The LDOS at the carbon atom which is furthest from the metal electrode displays a state distribution similar to that of the carbon atom in the contact region. The calculated total charge density of the SWNT bonded to the metal electrode, $E_{\mathrm{M}}$, i.e. $\rho\left[\mathrm{SWNT}+E_{\mathrm{M}}\right]$, and the difference charge density, $\Delta \rho=\rho\left[\mathrm{SWNT}+E_{\mathrm{M}}\right]-\rho[E]-\rho[\mathrm{SWNT}]$, show minute charge rearrangements. These results indicate that the weak Au electrode-SWNT interaction does not induce any significant changes in the electronic structure. The SCF electronic potential between the SWNT and the Au electrode, $V_{\mathrm{e}}(\mathbf{r})$, is presented on a vertical plane and also on a horizontal plane bisecting $\mathbf{s}$ in figures 33(b) and (c). The shaded area shows that the electronic potential energy at the contact yields a potential barrier $\Phi_{\mathrm{c}}=V_{\mathrm{e}}-E_{\mathrm{F}}>0$. At the contact midway between the SWNT and $\mathrm{Au}(100)$ surface, $\Phi_{\mathrm{c}}$ is calculated to be $\sim 3.9 \mathrm{eV}$-that is, comparable with the calculated work function $(\Phi \sim 5 \mathrm{eV})$ of the Au slab. Figure 33(d) shows the variation of $V_{\mathrm{e}}(z)$ on a line passing through the centre of the SWNT and perpendicular 

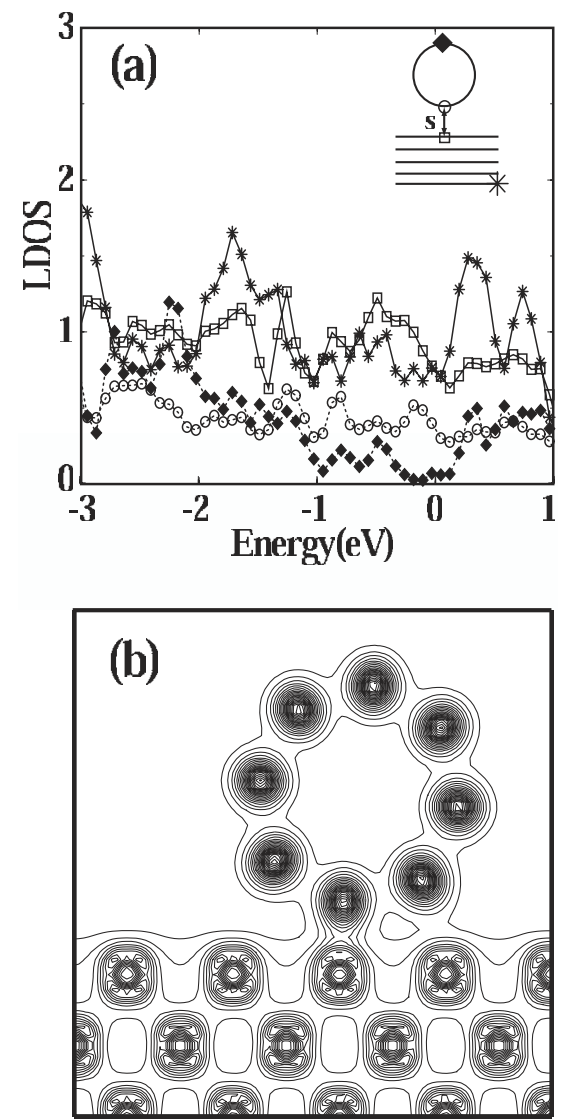

Figure 34. (a) Local densities of states (LDOSs) at different Mo and C atoms of the $(8,0)$ zigzag SWNT side-bonded to the Mo(110) surface. The locations of atoms are shown in the inset. The zero of energy is taken at the Fermi energy $E_{\mathrm{F}}$. State densities shown by empty circles and filled diamonds correspond to eight neighbouring carbon atoms, The others are for single atoms. (b) Contour plots of the total SCF charge density on a vertical plane. State densities shown by empty circles correspond to ten neighbouring carbon atoms. (Reproduced from [179].)

to the $\mathrm{Au}(100)$ surface. The effective potential barrier, $\Phi_{\mathrm{c}, \text { eff }}$, can be even higher owing to the increased confinement of electrons in the contact region. Therefore, electrons which are transferred from the metal to the s-SWNT have to tunnel through a potential barrier $\Phi_{\mathrm{c}, \text { eff }}(\mathbf{r})$.

The above situation is, however, different in the case of an s-SWNT side-bonded to the $\mathrm{Mo}(110)$ surface, as shown in figure 34. Upon relaxation, the tube has rotated slightly so C atoms tend to approach Mo atoms. The LDOS at the Mo atom interacting with the $\mathrm{C}$ atoms of the SWNT is different from that at the clean Mo(110) surface. Moreover, the LDOS at the carbon atom closest to the Mo surface has finite state density at $E_{\mathrm{F}}$. In particular, the peak near $E_{\mathrm{F}}$ is associated with the C-Mo bond states and hence may be attributed to the metal induced gap states (MIGS). The LDOS of the $\mathrm{C}$ atoms furthest from the contact has a band gap near $E_{\mathrm{F}}$. This situation indicates that the site of the SWNT forming contact is conducting, while the opposite site furthest from the contact remains semiconducting. Owing to the strong Mo-C bond, the spacing between the SWNT and the Mo electrode is smaller $(\mathbf{s}=1.96 \AA)$ than that with the Au electrode. The strong C-Mo bond formation is clarified by the calculated total SCF charge density in figure 34(b). The analysis of the difference charge density $\Delta \rho$ shows 
the strong charge transfer to the C-Mo bonds. The electronic potentials calculated on the horizontal plane bisecting $\mathbf{s}$ and also on a line from the SWNT to the Mo(110) surface through the contact show that $V_{\mathrm{e}}(\mathbf{r})<E_{\mathrm{F}}$ in the contact region. Accordingly, a potential barrier, $\Phi_{\mathrm{c}}$, does not form at the extended contact between the SWNT and the Mo electrode.

It appears that the above results distinguish two different types of electronic structure for two different electrodes. A large spacing and sizable potential barrier between the tube and metal electrode, $\Phi_{\mathrm{c}} \sim 3.9 \mathrm{eV}$, are characteristics of the contact made with the Au surface. This explains why the devices made from Au electrodes have high contact resistance. Here, weak coupling of electronic states may not lead to MIGS and the $(8,0)$ SWNT is identified as being semiconducting even after the contact has been set with the Au surface. Because of weak coupling and hence finite $\Phi_{\mathrm{c}}$, the s-SWNT-Au contact is reminiscent of the metal-oxidesemiconductor (MOS) junction. A small $\Phi_{\mathrm{B}}$ is estimated for the p-type character. Upon radial deformation, $\mathbf{s}$ decreases and eventually $\Phi_{\mathrm{c}}$ collapses. Similar features have been observed recently in STM studies using multiwall carbon nanotube tips and $\mathrm{Au}(111)$ samples [180]. However, $\Phi_{\mathrm{c}}$ has disappeared due to strong coupling between the states of the s-SWNT and $\operatorname{Mo}(110)$ [91]. The height of the Schottky barrier $\Phi_{B}$ that forms at the SWNT-Mo(110) junction having a finite contact region followed by a free s-SWNT depends on the position where the Fermi level is pinned in the gap of the s-SWNT. A crude estimate based on the LDOS suggests that $\Phi_{\mathrm{B}} \sim 0.4 \mathrm{eV}$ for the p-type character. The small cross section of the tube prevents us from identifying a band diagram across the diameter. But the bands are normally bent along the axis of the s-SWNT and the height of the barrier is monitored via the applied gate voltage. Finally, we note that the electronic properties of present metal-SWNT junctions, in particular the Fermi level pinning, exhibit marked differences from those of metal-Si heterostructures, which are known to be insensitive to the type of metal.

At this point, we also mention an interesting work by Buldum and $\mathrm{Lu}$ [181] on the intermolecular junctions of SWNTs. They found atomic scale characteristics in the quantum transport properties of junctions between two SWNTs. In particular, the contact resistance was strongly dependent on the local atomic configuration of the contact. The optimal electronic transport between nanotubes occurred when the surfaces of SWNTs were in atomic registry. However, as illustrated in figure 35, the contact resistance can vary over several orders of magnitude when the relative atomic configuration at the contact has been changed. Their predictions were confirmed experimentally [182]. It has been suggested that the monitoring of contact resistance may lead to new atomic scale switches, resistors and amplifiers.

A similar effect was predicted earlier for an $\mathrm{Al}$ chain, which is connected to two flat $\mathrm{Al}$ substrates to a weaker extent. It was found that the quantum conductance of the chain depends on the position of the $\mathrm{Al}$ atom (whether in a top site or a hollow site) where the chain is attached to the electrodes [183].

Metal nanorings. Metal nanorings are of particular interest, because they may lead to the realization of nanosolenoids on SWNTs. The small radius of a metallic nanoring wrapping the carbon nanotube may lead to interesting electromagnetic properties. The magnetic field $B$ at the centre of the ring can be expressed in terms of the quantized angular momentum $L_{z}$ of the electrons in the direction parallel to the tube axis

$$
B=\frac{\mu_{0} e L_{z}}{4 \pi m r^{3}}
$$

where $r$ is the radius of the nanoring. Taking the lowest possible value for $L_{z}$ and $r=5.9 \AA$, we estimate $B$ to be of the order of $100 \mathrm{G}$. The current in the metal ring that can induce such a high magnetic field is comparable to the current attained in suspended, monatomic 

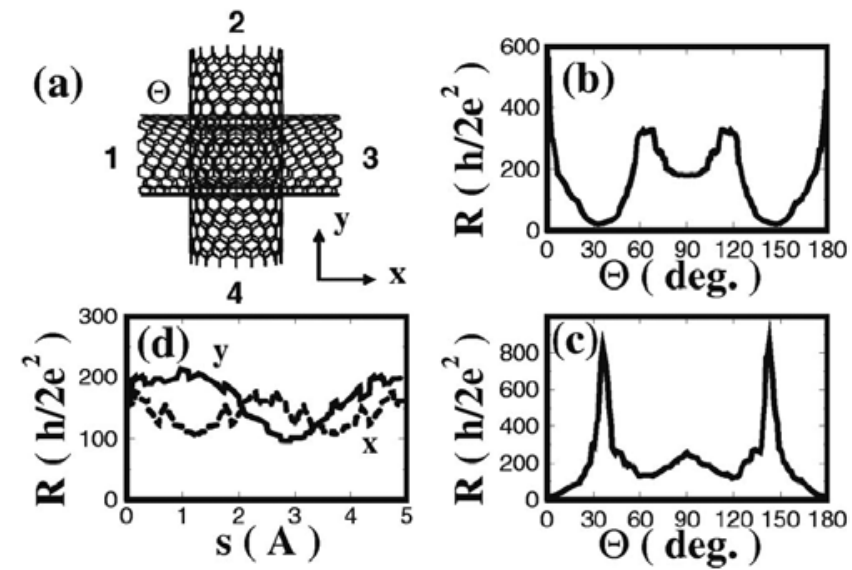

Figure 35. (a) A model of a four-terminal junction formed by crossing two nanotubes. The terminal labels, rotation angle, $\Theta$, and the translation directions are shown. The tubes are considered to be rigid. The current is passing between 1 and 4 and the voltage is measured between 2 and 3 . (b) The contact resistance of the $(18,0)-(10,10)$ junction as a function of the rotation angle $\Theta$. The tubes are in registry at $\Theta=30^{\circ}, 90^{\circ}, 150^{\circ}$. (c) The resistance of the $(10,10)-(10,10)$ junction. The tubes are in registry at $\Theta=0^{\circ}, 60^{\circ}, 120^{\circ}, 180^{\circ}$. (d) The resistance of the $(18,0)-(10,10)$ junction as a function of one tube relative to the other in the $x$ and $y$ directions. (Reproduced from [181].)

gold chains [88-90]. Relatively higher magnetic fields of the order of teslas can be induced by a higher current passing through a thick Ti based metal coating around the SWNT, or by increasing the number of turns and hence by forming a nanocoil. Miyamoto et al [184] have examined the chiral conductivity in bare $\mathrm{BC}_{2} \mathrm{~N}$ nanotubes. They estimated that a magnetic field of a few tenths of a tesla can be induced at the centre of the tube by assuming a relaxation time for carriers $\sim 50$ times larger than that in $\mathrm{Cu}$ and a homogeneous chiral current density confined to the tubule wall.

Metal nanorings winding the SWNT are also important, because they may allow the generation of persistent current at the nanoscale. Persistent currents in the nanoring can be started by sudden application of an external magnetic field. In this way it is possible to use a nanotube, with a ring at its end, as a local magnetic probe at the nanoscale. The persistent currents induced around a SWNT have been proposed as possible qubits in quantum computation by Kulik et al [185]. A superconducting ring may also be used for Schrödinger's cat experiments where one deals with the superposition of macroscopic quantum states [186]. The two supercurrent quantum states (clockwise and anticlockwise flow) sit in two separate quantum wells. It has been observed that a weak microwave, which does not break Cooper pairs, can cause quantum tunnelling between these two macroscopic states. In this kind of experiment the main problem is isolating the superconducting quantum interference device (SQUID) from the outside (nonquantum) environment and that is why isolated carbon nanotubes can be very useful.

Motivated by these exciting future applications, Bagci et al [109] studied the formation of Al coverage and Al nanorings around a $(8,0)$ SWNT. Since aluminium atoms have strong $\mathrm{Al}-\mathrm{Al}$ interaction, but not so strong Al-SWNT interaction, they tend to form clusters or nanoparticles (i.e. 3D islands) on the surface of the SWNT. However, Bagci et al showed that at special configurations both a stable nanoring and a metal tube can be formed. Moreover, these structures become stable even if the underlying SWNT is taken out. We first discuss the zigzag $\mathrm{Al}$ nanoring coverage which is obtained by placing $\mathrm{Al}$ atoms on top of carbon atoms 


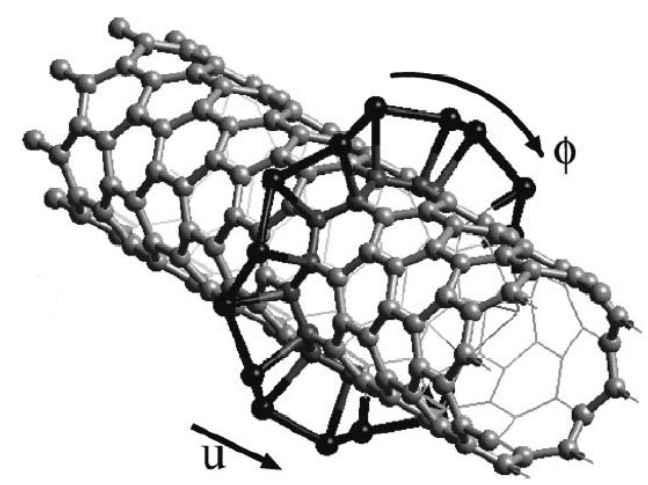

Figure 36. A view of the optimized structure of the Al zigzag nanoring formed on a $(8,0)$ SWNT. (Reproduced from [109].)

(T site), forming a zigzag ring (see figure 36). This structure includes $64 \mathrm{C}$ and $16 \mathrm{Al}$ atoms in the double unit cell. In this initial configuration the $\mathrm{Al}-\mathrm{Al}$ distance is $2.33 \AA$ and the angle of the $\mathrm{Al}-\mathrm{Al}-\mathrm{Al}$ bond is $\sim 137^{\circ}$. After structure optimization, the $\mathrm{Al}-\mathrm{Al}$ bond length is increased to $2.56 \AA$ and the $\mathrm{Al}-\mathrm{Al}-\mathrm{Al}$ bond angle is decreased to $124^{\circ}$, yielding the radius of the nanoring as $5.9 \AA$. A side view of the optimized structure of the Al nanoring wrapping the $(8,0)$ SWNT is illustrated in figure 36 . The binding energy of the $\mathrm{Al}$ nanoring is calculated to be $0.85 \mathrm{eV}$. The stability of the Al nanoring around the nanotube can be understood from the stable structures of planar Al monatomic chains. Recent studies on low dimensional structures of metals have revealed several stable atomic structures in one dimension (1D) [37, 94, 110, 187-191]. The final optimized structure of the zigzag Al nanoring around the $(8,0)$ nanotube has structural parameters similar to those of the planar zigzag structure [110], except that it is rolled on a cylinder. The SWNT initially serves as a template in the formation of the ring structure and also increases the stability of the ring by preventing transitions to other relatively more stable structures. Therefore, the Al nanoring around the SWNT is expected to be stable at room temperature. Interestingly, the nanoring is also stable by itself, since the positions of Al atoms do not change significantly upon discarding the underlying carbon nanotube.

The electronic properties of the zigzag $\mathrm{Al}$ nanoring system are also quite interesting. The electronic energy bands of the Al metal ring (without a SWNT) are derived from the dispersive bands of the flat zigzag Al chain [110]. When the flat zigzag Al chain is rolled into a ring, its bands are zone folded at the $\Gamma$ point and they appear as a number of discrete energy levels. For the case of the Al nanoring wrapping the nanotube, these states are mixed with the states of the nanotube and give rise to the bands and a density of states, which contributes a metallic behaviour to the combined $\mathrm{Al}$ nanoring and $(8,0)$ nanotube system. According to Mulliken analysis, 0.15 electrons are transferred from each Al atom to the SWNT. Most importantly, the $\mathrm{Al}$ ring is a conductor that incorporates two channels with an ideal ballistic quantum conductance of $4 e^{2} / h$.

Nanomagnets. In section 5.2, we showed that nanomagnets can form once the SWNT is fully covered or filled by the specific transition metal atoms. Therefore, first-principles calculations by Durgun et al $[102,103]$ present evidence that one can generate nanomagnets from SWNTs decorated by transition metal atoms (such as $\mathrm{Ti}$, Ta). From the spintronics and molecular magnetism point of view, the subject is extremely important and requires further study of the magnetization of transition metal atom covered SWNTs. Yang et al [111] performed 

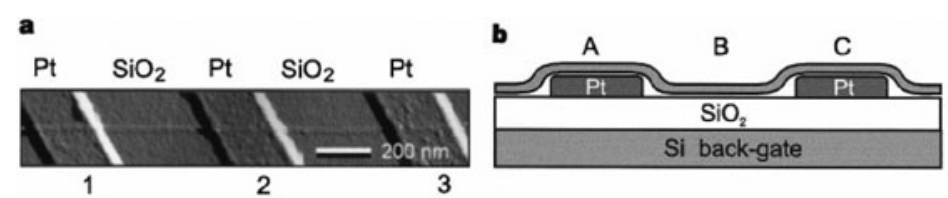

c
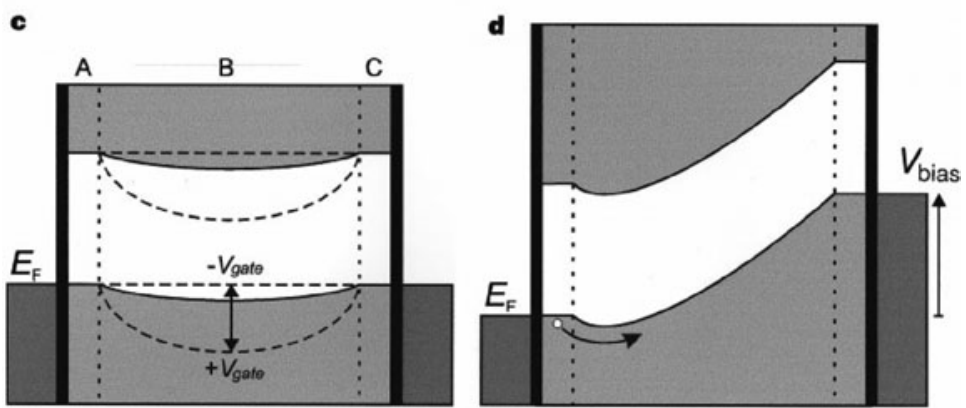

Figure 37. (a) A tapping mode AFM image of an individual carbon nanotube on top of three $\mathrm{Pt}$ electrodes. (b) A schematic side view of the FET device. A single s-SWNT is contacted by two electrodes. The $\mathrm{Si}$ substrate, which is covered by a layer of $\mathrm{SiO}_{2} 3000 \AA$ thick, acts as a back-gate. (c) The suggested band diagram of the device. The nanotube with a gap of $\sim 0.6 \mathrm{eV}$ is connected to the leads with Fermi energy $E_{\mathrm{F}}$ by tunnelling contacts, indicated by the black vertical bars. At $\mathrm{A}$ and $\mathrm{C}$, the valence band edge is pinned to the Fermi energy of the leads. Owing to a difference in work function between the tube and the electrodes, the bands bend towards lower energy in between the electrodes. For $V_{\text {gate }}>0$ the bands bend more strongly, leading to an insulating state; for $V_{\text {gate }}<0$ the bands are flattened, resulting in a metal-like conductance. (d) Application of a bias voltage results in suppression of the barrier. (Reproduced from [99].)

first-principles calculations of Co filled and Co coated $(9,0)$ tubes and found that these systems exhibit substantial magnetic moments $\left(1.4 \mu_{\mathrm{B}}\right.$ per Co atom) and have a very high spin polarization, $P=\left(D_{\downarrow}\left(E_{\mathrm{F}}\right)-D_{\uparrow}\left(E_{\mathrm{F}}\right)\right) /\left(D_{\downarrow}\left(E_{\mathrm{F}}\right)+D_{\uparrow}\left(E_{\mathrm{F}}\right)\right)(P=86 \%)$ for the spin valve effect. Dag et al [7] have obtained a significant net magnetic moment for Ti covered $(8,0),(9,0)$ and $(6,6)$ SWNTs.

\section{Devices fabricated using carbon nanotubes}

The objective of most of the effort made so far in the research on carbon nanotubes has been to discover a technological application. Nanoelectronic or molecular electronics considered as ultimate miniaturization [192] has attracted much of the interest. The realization of a singlemolecule device has been challenging due to difficulties in manipulating the molecule and achieving the electrical contact. Intensive laboratory work combined with theoretical studies during last decade has made remarkable advances in methods and techniques which were able to produce novel electronic devices based on carbon nanotubes. These devices have kept the great expectations for these materials alive and have continued to motivate further research. Some of these devices will be reviewed in this section.

\subsection{Transistors based on carbon nanotubes}

In 1998 Tans et al [99] achieved the fabrication of a field effect transistor (FET), which consists of a semiconducting SWNT connected to two metal electrodes. A semiconducting Si substrate covered with a $300 \mathrm{~nm}$ layer of thermally grown $\mathrm{SiO}_{2}$ was used as a back-gate (see figure 37). The nanotube was able to switch from a conducting to an insulating state at room temperature as 


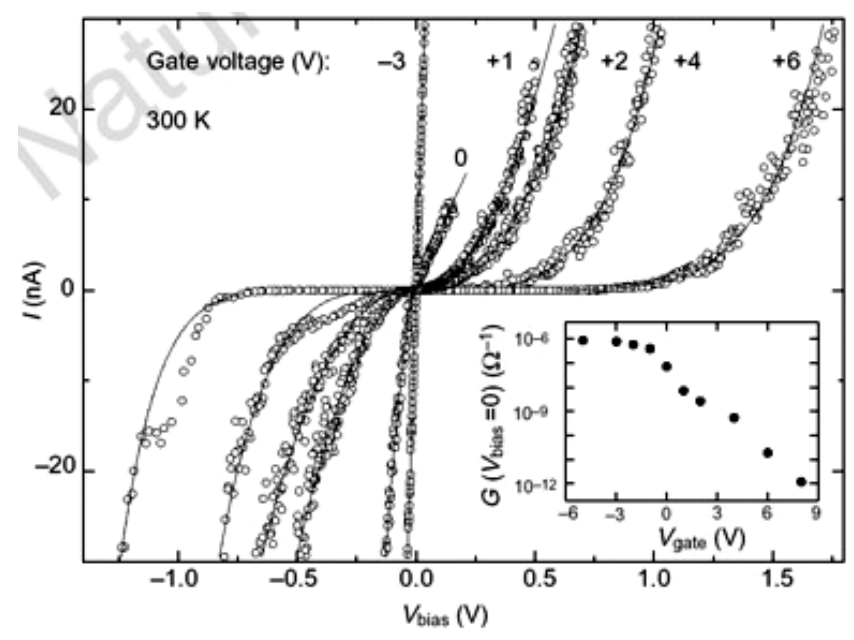

Figure 38. Two-probe $I-V_{\text {bias }}$ curves for various values of the gate voltage $\left(V_{\text {gate }}\right)$. Data were taken at room temperature and in vacuum $\left(\sim 10^{-4}\right.$ mbar) with the voltage applied to contacts 1 (drain) and 2 (source). A negative $V_{\text {gate }}$ leads to ohmic behaviour while a positive $V_{\text {gate }}$ results in a strong suppression of the current at low bias voltage and nonlinear $I-V_{\text {bias }}$ curves at higher bias. Inset: conductance at $V_{\text {bias }}=0$ as a function of $V_{\text {gate. }}$. The conductance through this single-molecule switch can be varied over at least six orders of magnitude and saturates at $10^{-6} \Omega^{-1}$ for negative $V_{\text {gate. }}$ (Reproduced from [99].)

a result of the voltage applied to the gate. As shown in figure 38, at $V_{\text {gate }}=0, I-V_{\text {bias }}$ exhibited a small nonlinearity. However, a pronounced gap-like nonlinearity developed around $V_{\text {bias }}=0$ with increasing $V_{\text {gate }}$. At negative $V_{\text {gate }}$, the $I-V_{\text {bias }}$ curve becomes linear with a resistance that saturates around $1 \mathrm{M} \Omega$. A change of $10 \mathrm{~V}$ in $V_{\text {gate }}$ resulted in modulation of the conductance by about six orders of magnitude at $V_{\text {bias }}=0$. According to the electronic structure and energy diagram of the FET modelled by Tans et al in figure 37(c) the difference in work function will result in bending of the bands of the semiconducting SWNT. A local polarization layer develops at the interface until the valence band edge of the SWNT aligns with the Fermi level of the metal electrode. The band bending length was estimated to be $400 \mathrm{~nm}$. It was assumed that the Fermi level is pinned at the valence band edge of the SWNT at the contact, since the FET has the same room temperature resistance of $\sim 1 \mathrm{M} \Omega$ and a similar temperature dependence. This behaviour of the device, namely a mild temperature dependence, has been attributed to the absence of a Schottky barrier. Since the proposed band diagram of the device was reminiscent of that of the barrier injection transit time diode (BARITT), its operation is qualitatively described in terms of the BARITT model. It has been argued that using special geometry, reducing the contact resistance and the thickness of $\mathrm{SiO}_{2}$, the device would allow a maximum frequency of the order of $10 \mathrm{THz}$. Also its gain can be raised by one order if the thickness of $\mathrm{SiO}_{2}$ is decreased from 3000 to $50 \AA$. It has also been foreseen that the integration of multiple devices into a circuit would be possible, by molecular self-assembly techniques.

Later, Zhou et al [98] measured $I-V$ characteristics of devices obtained with s-SWNTs contacted by $200 \AA$ thick Ni electrodes with $600 \AA$ thick Au on top. The lengths of the SWNTs between electrodes were less than $30 \mu \mathrm{m}$. Degenerately doped Si wafers with $5000 \AA$ thick thermally grown oxide on the surface were used as the substrate. The heavily doped substrate is conducting at low temperature and was used as a back-gate. Electrical transport through an s-SWNT involved thermal activation at high temperature and tunnelling behaviour through a 
reverse biased metal-SWNT junction at low temperature. Under high $V_{\text {bias }}, I-V$ characteristics of s-SWNTs exhibit pronounced asymmetry with respect to the bias polarity, as a result of local gating. Different samples exhibited $I-V$ characteristics resembling that of the Si based p metal-oxide-semiconductor field effect transistor (MOSFET). The positive voltage gain of $\sim 3$ recorded in this device was almost one order of magnitude higher than the maximum gain of $\sim 0.35$ obtained by Tans et al [99]. The transconductance was measured to be $\sim 200 \mathrm{nA} \mathrm{V}^{-1}$ which is two orders of magnitude higher than the similar results obtained by Martel et al [101]. These values indicate great progress.

Both transistors discussed above have been realized by the side bonding of SWNTs. These devices generally have high contact resistance and unipolar (p-type) transport characteristics. High contact resistance between the side-bonded s-SWNT and high work-function metal electrode is in accord with the first-principles calculations [179]. Martel et al [101] realized an end-bonded SWNT-metal contact. A FET was made of a semiconducting SWNT of $\sim 8000 \AA$ length and $14 \AA$ diameter contacting $\mathrm{TiC}$ and passivated with a uniform $\mathrm{SiO}_{2}$ layer. In contrast to the above planar devices, in the latter device the apparent barrier height for carrier injection is modulated by the gate field. This allowed the FET device to be ambipolar with low contact resistance for both n- and p-type conduction. Devices showing ohmic $I-V$ curves in both strong accumulation and inversion regimes have been attributed to the very small Schottky barrier at the contact. The low barriers for both electrons and holes have been attributed to the effective barrier heights being strongly modulated by the electrostatic field of the gate. Because of their quasi-one-dimensional structure the electronic behaviour of nanotube contacts is found to be sensitive to the electrostatics [193]. Fermi level pinning appears to be relatively unimportant for nanotubes end-bonded to TiC. The needle-like shape of the metallic carbide contacts focuses the electric field and hence the gate can induce a stronger electric field at the junction. As a result, whereas the true Schottky barrier height is as high as one expects (i.e. $\Phi_{\mathrm{B}}=E_{\mathrm{g}} / 2$ ), it is so thin that carriers can easily tunnel through the barrier.

The symmetry and narrowness of the Schottky barrier for both holes and electrons in a FET based on an s-SWNT have led to an interesting application. Injection of electrons and holes simultaneously through the narrow barriers at the source and drain contacts has been possible at room temperature. These injected carriers have recombined and emitted photons with energy $h v=E_{\mathrm{g}}$ and wavelength $\lambda \sim 0.8-1.5 \mu \mathrm{m}$. Because $\lambda$ depends on the $E_{\mathrm{g}}$ of the s-SWNT, the wavelength of the emitted light can be adjusted easily by using an s-SWNT with a different radius, or one can tune the emission to any wavelength by radial deformation. This new result holds promise that SWNTs can enable nanoscale photon sources for future optoelectronics and fibre optics [194].

The charge carrier mobility is crucial for the applications such as in few- and singleelectron memories and chemical/biochemical sensors. Instead of a Schottky barrier as in previous studies, the work by Durkop et al [195] exhibited an ohmic contact behaviour. They presented measurements on long, ohmically contacted nanotube devices, showing field effect mobility in semiconducting SWNT transistors as high as $79000 \mathrm{~cm}^{2} \mathrm{~V}^{-1} \mathrm{~s}^{-1}$ at room temperature, and the intrinsic mobility in s-SWNTs is even higher. These measured values exceed those of any other semiconductor at room temperature.

Beyond the single FET, research on SWNT based electronics has focused on the integration of FETs on a chip, since the advance requires assembly strategies that allow precise localization and interconnection. Soh et al [197] achieved the synthesis of SWNTs by chemical vapour deposition (CVD) of methane at controlled locations on a substrate using patterned catalytic islands. The CVD synthesis and microfabrication techniques were combined to produce a large number of ohmically contacted nanotube devices with controllable length, placed on a Si wafer with $\sim 1 \mu \mathrm{m}$ thick thermally grown $\mathrm{SiO}_{2}$. It has been argued that this fabrication technique 
allows large numbers of integrated nanotube circuits to be built reliably with a contact resistance of $\sim 10 \mathrm{k} \Omega$ (or $20 \mathrm{k} \Omega$ two-terminal resistance in the ballistic regime). Later Franklin et al [198] devised a method for reliable integration of long suspended SWNTs into electrically addressable devices, which involves patterned growth of SWNTs to bridge predefined Mo electrodes. This approach affords SWNT based devices without any postgrowth processing. In the course of active research towards nanoelectronics, Bachtold et al [199] demonstrated logic circuits with FETs based on SWNTs. Their transistors show device characteristics such as high gain $(>10)$, a large on-off ratio $\left(>10^{5}\right)$ and room temperature operation.

Much recently, Keren et al [196] reported the realization of a self-assembled carbon nanotube FET operating at room temperature where a DNA scaffold molecule provides the address for precise localization of an s-SWNT as well as a template for the extended metallic wires contacting it. In order to get the transistor to self-assemble, they attached a SWNT onto a specific site on a DNA strand, by binding together with proteins. They then made gold nanowire out of DNA molecules at each end of the nanotube. The device could be switched on and off by applying voltage to it.

\subsection{Chemical sensors}

As discussed in section 3, the adsorption of atoms or molecules such as $\mathrm{NO}, \mathrm{NH}_{3}$ and $\mathrm{O}_{2}$ on s-SWNTs has been reported to change the electrical resistance and similar properties. It has been suggested that this effect of carbon nanotubes can be used in chemical sensors to detect specific molecules in the environment $[62,63]$. Owing to their bulky size and high power consumption, the miniaturization of chemical sensors is, in fact, very important for various applications. Modi et al [200] achieved production of a miniaturized gas ionization sensor a few microns in length using MWNTs. The sharp tips of MWNTs generate very high electric field at relatively low voltages, lowering breakdown voltages several times in comparison to those of traditional electrodes. This feature enables compact, battery powered and safe operation of such sensors. In this sensor, controlled dc voltage is applied between an anode consisting of vertically aligned MWNT film and a cathode made from Al sheet. A MWNT film (consisting of MWNTs 250-300 $\AA$ in diameter, $\sim 30 \mu \mathrm{m}$ long, with $\sim 500 \AA$ separation) was grown by CVD on a $\mathrm{SiO}_{2}$ substrate. An individual MWNT, attached to the substrate in the head-on position, has a tip radius of $150 \AA$, which, in turn generates a very high nonlinear electric field. In this way the formation of a corona is promoted at relatively low voltage, which can be provided by special batteries. The breakdown voltage was found to become lower as the interelectrode spacing was reduced. The breakdown voltage went down from $280 \mathrm{~V}$ (at $150 \mu \mathrm{m}$ ) to $130 \mathrm{~V}$ (at $25 \mu \mathrm{m}$ separation). Such voltages can be easily obtained by series connection of carbon-zinc batteries. That makes the sensor portable. In the operation of the sensor, it is found that while the breakdown voltage is independent of the gas concentration, the current discharge is linearly dependent on the concentration. This sensor and the underlying technique that it uses are very convenient, because: (i) the precise breakdown voltage provides the fingerprint for the gas to be identified; (ii) by monitoring the self-sustaining discharge current, the gas concentration can be determined; (iii) the response time is short [200].

\section{Conclusions}

Because of growing interest in nanotechnology and the quest for novel nanodevices, study of carbon nanotubes has seen a tremendous explosion of work. Despite the large number of very important research works, the scope of this review has been focused on a limited field covering atomic and electronic structure of single-wall carbon nanotubes. 
In this review article, various physical properties of carbon nanotubes and specific applications using these properties have been investigated. Our discussions have been guided by first-principles calculations within density functional theory, which were proven to be appropriate and to yield accurate predictions on the atomic and electronic structure of carbon nanotubes.

It has been shown that bare nanotubes have many important properties which are suitable for use in potential technological applications. That a $(n, m)$ SWNT can be metallic or semiconducting depending on $n$ and $m$ offers a wide range of options. Moreover, these options are multiplied several times by the functionalization of tubes. Here we dealt with specific cases wherein the properties of the nanotube undergo a dramatic change upon physisorption of foreign atoms. Not only functionalization but also radial deformation has been seen to modify the nanotube properties. A semiconducting nanotube can be metallic under a radial strain which changes the circular cross section into an elliptical one. Even more interesting is that these changes can be tuned reversibly. Finally, we discussed how properties of bare nanotubes as well as those induced by functionalization or radial deformation can be exploited for the design of new molecular devices. Transistors and chemical sensors fabricated by using carbon nanotubes, as explained at the end, show that carbon nanotubes are indeed nanostructures which may potentially allow several technological applications.

\section{Acknowledgments}

This work was partially supported by the National Science Foundation under Grant No INT0115021 and TÜBİTAK under Grant No TBAG-U/13(101T010).

\section{References}

[1] Iijima S 1991 Nature 35456 Iijima S, Ichihashi T and Ando Y 1992 Nature 356776

[2] Dresselhaus M S, Dresselhaus G and Eklund P C 1996 Science of Fullerenes and Carbon Nanotubes (San Diego, CA: Academic)

[3] Saito R, Dresselhaus G and Dresselhaus M S 1998 Physical Properties of Carbon Nanotubes (London: Imperial College)

[4] Zhao X, Ando Y, Liu Y, Jinno M and Suzuki T 2003 Phys. Rev. Lett. 90187401

[5] Yu M F et al 1998 Science 287637

[6] Salvetat J P et al 1999 Appl. Phys. A 69255

[7] Dag S, Durgun E and Ciraci S 2004 Phys. Rev. B 69 121407(R)

[8] Li F, Xia X, Zhao M, Liu X, Huang B, Tan Z and Ji Y 2004 Phys. Rev. B 69165415

[9] Nevidomskyy A H, Csanyi G and Payne M C 2003 Phys. Rev. Lett. 91105502

[10] Dresselhaus M S, Dresselhaus G and Saito R 1992 Phys. Rev. B 456234

[11] Hamada N, Sawada S and Oshiyama A 1992 Phys. Rev. Lett. 681579

[12] Mintmire J W, Dunlap B I and White C T 1992 Phys. Rev. Lett. 68631

[13] White C T, Robertson D H and Mintmire J W 1993 Phys. Rev. B 475485

[14] Ciraci S, Buldum A and Batra I 2001 J. Phys.: Condens. Matter 13537

[15] Frank S, Poncharal P, Wang Z L and Heer W A 1998 Science 2801744

[16] Ouyang M, Huang J, Cheung C L and Lieber C M 2001 Science 292702

[17] Kim W, Choi H C, Shim M, Li Y, Wang D and Dai H 2002 Nano Lett. 2703

[18] Gülseren O, Yildirim T and Ciraci S 2002 Phys. Rev. 65153405

[19] Charlier J C, Ebbesen T W and Lambin Ph 1996 Phys. Rev. B 5311108

[20] Chico L, Lopez Sancho M P and Munoz M C 1998 Phys. Rev. Lett. 811278

[21] Collins P G, Zettl A, Bando H, Thess A and Smalley R E 1997 Science 278100

[22] Bockrath M, Cobden D H, McEuen P L, Chopra N G, Zettl A, Thess A and Smalley R E 1997 Science 275 1922

[23] Bezryadin A, Verschueren A R M, Tans S J and Dekker C 1998 Phys. Rev. Lett. 804036 
[24] Kiliç Ç, Ciraci S, Gülseren O and Yildirim T 2000 Phys. Rev. B 6216345

[25] Sim H S, Park C J and Chang K J 2001 Phys. Rev. B 63073402

[26] Gülseren O, Yildirim T and Ciraci S 2001 Phys. Rev. Lett. 87116802

[27] Wildöer J W G, Venema L C, Rinzler A G, Smalley R E and Dekker C 1998 Nature 39159

[28] Venema L C, Janssen J W, Buitelaar M R, Wildöer J W G, Lemay S G, Kouwenhoven L P and Dekker C 2000 Phys. Rev. B 625238

[29] Odom T W, Huang J, Kim P and Lieber C M 1998 Nature 39162

[30] Odom T W, Huang J, Kim P and Lieber C M 2000 J. Phys. Chem. B 1042794

[31] Blase X, Benedict L X, Shirley E L and Louie S G 1994 Phys. Rev. Lett. 721878

[32] Kane C L and Mele E J 1997 Phys. Rev. Lett. 781932

[33] Kleiner A and Eggert S 2001 Phys. Rev. B 63073408

[34] Kleiner A and Eggert S 2001 Phys. Rev. B 64113402

[35] Payne M C, Teter M P, Allen D C, Arias T A and Joannopoulos J D 1992 Rev. Mod. Phys. 641045

[36] Perdew J P and Wang Y 1992 Phys. Rev. B 466671

Perdew J P, Chevary J A, Vosko S H, Jackson K A, Pederson M R, Singh D J and Fiolhais C 1992 Phys. Rev. B 466671

[37] Sanchez-Portal D, Artacho E, Soler J M, Rubio A and Ordejon P 1999 Phys. Rev. B 5912678

[38] Robertson D H, Brenner D W and Mintmire J W 1992 Phys. Rev. B 4512592

[39] Tibbets G G 1984 J. Cryst. Growth 66632

[40] Kudin K N, Scuseria G E and Yakobson B I 2001 Phys. Rev. B 64235406

[41] Yorikawa H and Muramatsu S 1995 Solid State Commun. 94435

[42] Yorikawa H and Muramatsu S 1995 Phys. Rev. B 522723

[43] Gülseren O, Yildirim T, Ciraci S and Kiliç Ç 2002 Phys. Rev. B 65155410

[44] Park C J, Kim Y H and Chang K J 1999 Phys. Rev. B 6010656

[45] Dillon A C, Jones K M, Bekkadahl T A, Kiang C H, Bethune D S and Heben M J 1997 Nature 386377

[46] Liu C, Fan Y Y, Liu M, Cong H T, Cheng H M and Dresselhaus M S 1999 Science 2861127

[47] Brown C M, Yildirim T, Neumann D A, Heben M H, Gennett T, Dillon A C, Alleman J L and Fisher J E 1999 Chem. Phys. Lett. 329956

[48] Ahn C C, Ye Y, Ratnakumar B V, Witham C, Bowman R C and Fultz B 1998 Appl. Phys. Lett. 73378

[49] Chen C C, Wu X, Lin J and Tan K L 1999 Science 28591

[50] Wang Q, Challa S R, Sholl D S and Johnson J K 1999 Phys. Rev. Lett. 82956

[51] Gordillo R C, Boronat J and Casulleras J 2000 Phys. Rev. Lett. 852348

[52] Simonyan V V, Diep P and Johnson J K 1999 J. Chem. Phys. 1119778

[53] Ma Y, Xia Y, Zhao M, Wang R and Mei L 2001 Phys. Rev. B 63115422

[54] Tada K, Furuya S and Watanabe K 2001 Phys. Rev. B 63155405

[55] Yildirim T, Gülseren O and Ciraci S 2001 Phys. Rev. B 64075404

[56] Srivastava D, Brenner D W, Schall J D, Ausman K D, Yu M and Ruoff R S 1999 J. Phys. Chem. B 1034330

[57] Gülseren O, Yildirim T and Ciraci S 2002 Phys. Rev. B 66121401

[58] Andriotis A N, Menon M, Srivastava D and Froudakis G 2001 Phys. Rev. B 64193401

[59] Landauer R 1987 Z. Phys. B 68217

[60] Chan S P, Chen G, Gong X G and Liu Z F 2001 Phys. Rev. Lett. 87205502

[61] White C T, Robertson D H and Mintmire J W 1993 Phys. Rev. B 475485

[62] Kong J, Franklin N R, Zhou C, Chapline M G, Peng S, Cho K and Dai H 2000 Science 287622

[63] Collins P G, Bradley K, Ishigami M and Zettl A 2000 Science 2871802

[64] Dean K A and Chalamala B R 1999 J. Appl. Phys. 853832

Dean K A and Chalamala B R 2000 Appl. Phys. Lett. 76375

[65] Ajayan P M, Ebbesen T W, Ichihashi T, IIjima S, Tanigaki K and Hiura H 1993 Nature 362522

[66] Ebbesen T W, Ajayan P M, Hiura H and Tanigaki K 1994 Nature 367519

[67] Morishita K and Takarada T 1997 Carbon 35977

[68] Jhi S H, Louie S G and Cohen M L 2000 Phys. Rev. Lett. 851710

[69] Sorescu D C, Jordan K D and Avouris P 2001 J. Phys. Chem. B 10511227

[70] Zhu X Y, Lee S M, Lee Y H and Frauenheim T 2000 Phys. Rev. Lett. 852757

[71] Moon C-Y, Kim Y-S, Lee E-C, Jin Y-G and Chang K J 2002 Phys. Rev. B 65155401

[72] Park N, Han S and Ihm J 1999 Phys. Rev. B 64125401

[73] Mann D J and Halls M D 2002 J. Chem. Phys. 1169014

[74] Ricca A and Drosco J A 2002 Chem. Phys. Lett. 362217

[75] Dag S, Gülseren O, Yildirim T and Ciraci S 2003 Phys. Rev. B 67165424

[76] Giannozzi P, Car R and Scoles G 2003 J. Chem. Phys. 1181003 
[77] Dag S, Gülseren O and Ciraci S 2003 Chem. Phys. Lett. 3801

[78] Lee S M, Lee Y H, Hwang Y G, Hahn J R and Kang H 1999 Phys. Rev. Lett. 82217

[79] Lifshitz E M 1956 Zh. Eksp. Teor. Fiz. 2994 Lifshitz E M 1956 Sov. Phys._JETP 273 (Engl. Transl.)

[80] Israelachvili J N 1985 Intermolecular and Surface Forces (London: Academic)

[81] Ciraci S, Tekman E, Baratoff A and Batra I P 1992 Phys. Rev. B 4610411

[82] Halgren T A 1992 J. Am. Chem. Soc. 1147827

[83] Ulbricht H, Moos G and Hertel T 2002 Phys. Rev. B 66075404

[84] Derycke V, Martel R, Appenzeller J and Avouris Ph 2002 Appl. Phys. Lett. 802273

[85] Delaney P, Choi H J, Ihm J, Louie S G and Cohen M L 1998 Nature 391466 Delaney P, Choi H J, Ihm J, Louie S G and Cohen M L 1999 Phys. Rev. B 607899

[86] Chan S P, Chen G, Gong X G and Liu Z F 2003 Phys. Rev. Lett. 90086403

[87] Chiang I V, Brinson B E, Smalley R E, Magrave J L and Hauge H 2001 J. Phys. Chem. B 1051157

[88] Agraït N, Rodrigo J G and Vieira S 1993 Phys. Rev. B 4712345

[89] Ohnishi H, Kondo Y and Takayanagi K 1998 Nature 395783

[90] Yanson A I, Bollinger G R, Brom H E, Agräit N and Ruitenbeek J M 1998 Nature 395783

[91] Ciraci S and Tekman E 1989 Phys. Rev. B 40 R11969 Tekman E and Ciraci S 1991 Phys. Rev. B 437145

[92] Buldum A, Ciraci S and Fong C Y 2000 J. Phys.: Condens. Matter 123349 Buldum A, Leitner D M and Ciraci S 1999 Europhys. Lett. 47208

[93] Ozpineci A and Ciraci S 2001 Phys. Rev. B 63125415

[94] Gülseren O, Ercolesi F and Tosatti E 1998 Phys. Rev. Lett. 803775 Tosatti E and Prestipino S 2000 Science 289561

[95] Yao Z, Kane C L and Dekker C 2000 Phys. Rev. Lett. 844613

[96] Dai H, Wong E W, Lu Y Z, Fan S and Lieber C M 1995 Nature 375769 Han W Q, Fan S S and Li Q Q 1997 Science 2771287

[97] Zhang Y and Dai H 2000 Appl. Phys. Lett. 773015 Zhang Y, Franklin N W, Chen R J and Dai H 2000 Chem. Phys. Lett. 33135

[98] Zhou C, Kong J and Dai H 2000 Phys. Rev. Lett. 845604

[99] Tans S J, Verscheuren A R M and Dekker C 1998 Nature 39349 Bachtold A, Hadley P, Nakanishi T and Dekker C 2001 Science 2941317 Nakanishi T, Bachtold A and Dekker C 2002 Phys. Rev. B 66073307

[100] Soh H T, Quate C, Morpurgo A F, Marcus C M, Kong J and Dai H 1999 Appl. Phys. Lett. 75627 Zhou C, Kong J and Dai H 2000 Appl. Phys. Lett. 761597

[101] Martel R, Derycke V, Lavoie C, Appenzeller J, Chan K K, Tersoff J and Avouris Ph 2001 Phys. Rev. Lett. 87 256805

Avouris Ph, Martel R, Derycke V and Appenzeller J 2002 Physica B 3236

Martel R, Schmidt T, Shea H R, Hertel T and Avouris Ph 1998 Appl. Phys. Lett. 732447

[102] Durgun E, Dag S, Bagci V M K, Gülseren O, Yildirim T and Ciraci S 2003 Phys. Rev. B 67 201401(R)

[103] Durgun E, Dag S, Ciraci S and Gülseren O 2004 J. Phys. Chem. B 108575

[104] Zhang X X, Wen G H, Huang S, Dai L, Gao R and Wang Z L 2001 J. Magn. Magn. Mater. 231 L9 Satishkumar B C, Govindaraj A, Vanitha P V, Raychaudhuri A K and Rao C N R 2002 Chem. Phys. Lett. 362 301

[105] Winter M, Besenhard J O, Spahr M E and Novac M P 1998 Adv. Mater. 107259

[106] Gao B, Kleinhammer A, Tang X P, Bower C, Fleming L, Wu Y and Zhou O 1999 Chem. Phys. Lett. 3071536

[107] Zhao J, Buldum A, Han J and Lu J P 2000 Phys. Rev. Lett. 851706

[108] Yang C-K, Zhao J and Lu J P 2002 Phys. Rev. B 66 041403(R)

[109] Bagci V M K, Gülseren O, Yildirim T, Gedik Z and Ciraci S 2002 Phys. Rev. B 66045409

[110] Sen P, Ciraci S, Buldum A and Batra I P 2001 Phys. Rev. B 64195420

[111] Yang C-K, Zhao J and Lu J P 2003 Phys. Rev. Lett. 90257203

[112] Treacy M M J, Ebbesen T W and Gibson J M 1996 Nature 381678

[113] Ruoff R S, Tersoff J, Lorents D C, Subramoney S and Chan B 1993 Nature 364514

[114] Chopra N G, Benedict L X, Crespi V H, Cohen M L, Louie S G and Zettl A 1995 Nature 377135

[115] Rochefort A, Avouris P, Lesage F and Salahub D R 1998 Chem. Phys. Lett. 29745

[116] Rochefort A, Avouris P, Lesage F and Salahub D R 1999 Phys. Rev. B 6013824

[117] Liu L, Jayanthi C S, Tang M, Wu S Y, Tombler T W, Zhou C, Alexseyev L, Kong J and Dai H 2000 Phys. Rev. Lett. 844950

[118] Tombler T W, Zhou C, Alexseyev L, Kong J, Dai H, Liu L, Jayanthi C S, Tang M and Wu S 2000 Nature 405 769 
[119] Chesnokov S A, Nalimova V A, Rinzler A G, Smalley R E and Fischer J E 1999 Phys. Rev. Lett. 82343

[120] Lambert P E, Zhang P and Crespi V 2000 Phys. Rev. Lett. 842453

[121] Minot E D, Yaish Y, Sazonova V, Park J-Y, Brink M and McEuen P L 2003 Phys. Rev. Lett. 90156401

[122] Salvetat J P, Briggs G A D, Bonard J, Bacsa R R, Kulik A J, Stöckli T, Burnham N A and Forró L 1999 Phys. Rev. Lett. 82944

[123] Yildirim T, Gülseren O, Kiliç Ç and Ciraci S 2000 Phys. Rev. B 6212648

[124] Yang L, Anantram M P, Han J and Lu J P 1999 Phys. Rev. B 6013874

[125] Yang L and Han J 2000 Phys. Rev. Lett. 85154

[126] Shen W, Jiang B, Han B S and Xie S 2000 Phys. Rev. Lett. 843634

[127] Lordi V and Yao N 1998 J. Chem. Phys. 1092509

[128] Yu M F, Kowalewski T and Ruoff R S 2000 Phys. Rev. Lett. 851456

[129] Mazzoni M S C and Chacham H 2000 Appl. Phys. Lett. 761561

[130] Öztürk Y and Ciraci S 2004 at press

[131] Rao A M et al 1997 Appl. Phys. A 64231

[132] Venkateswaren U D, Rao A M, Richter E, Menon M, Rinzler A, Smalley R E and Eklund P C 1999 Phys. Rev. B 5910928

Sood A K, Teredesai P V, Muthu D V S, Sen R, Gavindaraj A and Rao C N 2000 Chem. Phys. Lett. 319296 Teredesai P V, Sood A K, Muthu D V S, Sen R, Govindaraj A and Rao C N R 2000 Chem. Phys. Lett. 310296

[133] Peters M J, McNeil L E, Lu J P and Kuhn D 2000 Phys. Rev. B 615939

[134] Tang J, Qin L C, Sasaki T, Yudasaka M, Matsushita A and Iijima S 2000 Phys. Rev. Lett. 851887

[135] Pernas P L, Martin-Rodero A and Flores F 1990 Phys. Rev. B 418553

[136] Tian W and Datta S 1994 Phys. Rev. B 495097

[137] de Parga A L V, Hernan O S, Miranda R, Yeyati A L, Mingo N, Martin-Rodero A and Flores F 1998 Phys. Rev. Lett. 80357

[138] Emberley E G and Kirczenow G 1999 Phys. Rev. B 606028

[139] Brandbyge M, Kobayashi N and Tsukada M 1999 Phys. Rev. B 6017064

[140] Mehrez H, Taylor J, Guo H, Wang J and Roland C 2000 Phys. Rev. Lett. 842682

[141] Roland C, Nardelli M B, Wang J and Guo H 2000 Phys. Rev. Lett. 842921

[142] Wei Y, Wang J, Guo H, Mehrez H and Roland C 2001 Phys. Rev. B 63195412

[143] Mehrez H, Guo H, Wang J and Roland C 2001 Phys. Rev. B 63245410

[144] Nardelli M B 1999 Phys. Rev. B 607828

[145] Orlikowski D, Mehrez H, Taylor J, Guo H, Wang J and Roland C 2001 Phys. Rev. B 63155412

[146] Chico L, Benedict L X, Louie S G and Cohen M L 1996 Phys. Rev. B 542600

[147] Wan C C, Mozos J L, Taraschi G, Wang J and Guo H 1997 Appl. Phys. Lett. 71419

[148] Wan C C, Mozos J L, Wang J and Guo H 1997 Phys. Rev. B 5513393

[149] Wang J, Guo H, Mozos J L, Wan C C, Taraschi G and Zheng Q 1998 Phys. Rev. Lett. 804277

[150] Taraschi G, Mozos J L, Wan C C, Guo H and Wang J 1998 Phys. Rev. B 5813138

[151] Mozos J L, Wan C C, Taraschi G, Wang J and Guo H 1997 Phys. Rev. B 564351

[152] Choi H J and Ihm J 1999 Phys. Rev. B 592267

[153] Choi H J, Ihm J, Yoon Y G and Louie S G 1999 Phys. Rev. B 6014009

[154] Choi H J, Ihm J, Louie S G and Cohen M L 2000 Phys. Rev. Lett. 842917

[155] Lang N D 1995 Phys. Rev. B 525335

[156] Hirose K and Tsukada M 1995 Phys. Rev. B 515278

[157] Lang N D and Avouris P 1998 Phys. Rev. Lett. 813515

[158] Yaliraki S N, Kemp M and Ratner M A 1999 J. Am. Chem. Soc. 1213428

[159] Di Ventra M, Pantelides S T and Lang N D 2000 Phys. Rev. Lett. 84979

[160] Landman U, Barnett R N, Scherbakov A G and Avouris P 2000 Phys. Rev. Lett. 851958

[161] Taylor J, Guo H and Wang J 2001 Phys. Rev. B 63245407

[162] Brandbyge M, Mozos J L, Ordejon P, Taylor J and Stokbro K 2002 Phys. Rev. B 65165401

[163] Palacios J J, Perez-Jimenez A J, Louis E, SanFabian E and Verges J A 2002 Phys. Rev. B 66035322

[164] Taylor J, Guo H and Wang J 2001 Phys. Rev. B 63121104

[165] Larade B, Taylor J, Mehrez H and Guo H 2001 Phys. Rev. B 64075420

[166] Roland C, Meunier V, Larade B and Guo H 2002 Phys. Rev. B 66035332

[167] Taylor J, Brandbyge M and Stokbro K 2002 Phys. Rev. Lett. 89138301

[168] Mehrez H, Wlasenko A, Larade B, Taylor J, Grutter P and Guo H 2002 Phys. Rev. B 65195419

[169] Sanvito S, Kwon Y-K, Tománek D and Lambert C J 2000 Phys. Rev. Lett. 841974

[170] Palacios J J, Pérez-Jiménez A J, Louis E, SanFabián E and Vergés J A 2003 Phys. Rev. Lett. 90106801

[171] Kaun C-C, Larade B, Mehrez H, Taylor J and Guo H 2002 Phys. Rev. B 65205416 
[172] Saito R, Dresselhaus G and Dresselhaus M S 1996 Phys. Rev. B 532044

[173] Chico L, Crespi V H, Benedict L X, Louie S G and Cohen M L 1996 Phys. Rev. Lett. 76971

[174] Esaki L (ed) 1991 Highlights in Condensed Matter Physics and Future Prospects (NATO Advanced Science Institute vol 285) (New York: Plenum)

[175] Colombo L, Resta R and Baroni S 1991 Phys. Rev. B 445572

[176] Gülseren O, Yildirim T and Ciraci S 2003 Phys. Rev. B 68115419

[177] Rochefort A, Di Vientra M and Avouris P 2001 Appl. Phys. Lett. 782521

[178] Heinze S, Tersoff J, Martel R, Derycke V, Appenzeller J and Avouris Ph 2002 Phys. Rev. Lett 89106801

[179] Dag S, Gülseren O, Yildirim T and Ciraci S 2003 Appl. Phys. Lett. 833180

[180] Luna M et al 2003 Ultramicroscopy 9683

[181] Buldum A and Lu J P 2001 Phys. Rev. 63161403

[182] Paulson S, Helser A, Nardelli M B, Taylor R M, Falvo M R, Superfine R and Washburn S 2000 Science 290 1742

[183] Mehrez H, Ciraci S and Batra I P 1997 Phys. Rev. B 55 R1981

[184] Miyamoto Y, Louie S G and Cohen M 1996 Phys. Rev. Lett. 762121

Miyamoto Y 1996 Phys. Rev. B 5411149

Miyamoto Y, Rubio A, Louie S G and Cohen M L 1999 Phys. Rev. B 6013885

[185] Kulik I O, Hakioglu T and Barone A 2002 Eur. Phys. J. B 30219

[186] Friedman J R, Patel V, Chen W, Tolpygo S K and Lukens J E 2000 Nature 40643

[187] Mehrez H and Ciraci S 1997 Phys. Rev. B 5612632

[188] Tosatti E, Prestipino S, Kostlmeier S, Dal Corso A and Di Tolla F D 2001 Science 291288

[189] Okamoto M and Takayanagi K 1999 Phys. Rev. B 607808

[190] Tolla F D, Corsa A D, Torres J A and Tosatti E 2000 Surf. Sci. 454-456 947

[191] Häkkinen H, Barnett R N, Scherbakov A G and Landman U 2000 J. Phys. Chem. B 1049063

[192] Aviran A and Ratner M A 1974 Chem. Phys. Lett. 29277

[193] Leonard F and Tersoff J 2000 Phys. Rev. Lett. 844693

[194] Avouris Ph 2003 Science 300783

[195] Durkop T, Getty S A, Cobas E and Fuhrer S 2004 Nano Lett. 435

[196] Keren K, Berman R S, Buchstab E, Sivan U and Braun E 2003 Science 3021380

[197] Soh H T, Quate C F, Morpugo A F, Marcus C M, Kong J and Dai H 1999 Appl. Phys. Lett. 75627

[198] Fraklin N R, Wang Q, Tombler T W, Javey A, Shim M and Dai H 2002 Appl. Phys. Lett. 81913

[199] Bactold A, Hadley P, Nakanishi T and Dekker C 2001 Science 2971317

[200] Modi A, Koratkar N, Lass E, Wel B and Ajayan P M 2003 Nature 424171 